\title{
Trimethyl borate-catalyzed, solvent-free reductive amination
}

P. Veeraraghavan Ramachandran,* Shivani Choudhary, and Aman Singh

Department of Chemistry, 560 Oval Drive, Purdue University West Lafayette, Indiana 47907-2084; Fax: 765494 0239; Tel: 765494 5303; E-mail: chandran@purdue.edu

\section{Supporting Information}

Table of Contents

Table 1: Optimization of reaction conditions S2

NMR Data S3

Green Matrix calculation S35 
Table S1. Optimization of reaction conditions for reductive amination of benzaldehyde (2a) with benzylamine (3a) using ammonia borane (1): Examination of Lewis acids and their equivalents, solvents and reaction time on the yield of dibenzylamine (4aa)

\begin{tabular}{|c|c|c|c|c|c|}
\hline Entry & Lewis acid & $\begin{array}{l}\text { Lewis acid } \\
\text { (equiv.) }\end{array}$ & Solvent & $\begin{array}{l}\text { Time } \\
\text { (h) }\end{array}$ & $\begin{array}{l}\text { Yield } \\
(\%)^{a}\end{array}$ \\
\hline & None & & $\mathrm{MeOH}$ & 4 & 51 \\
\hline & $\operatorname{Ti}(\mathrm{O}-i-\mathrm{Pr})_{4}^{b}$ & 1 & $\mathrm{THF}$ & 4 & 94 \\
\hline & $\mathrm{AcOH}$ & 1 & $\mathrm{H}_{2} \mathrm{O}$ & 12 & 17 \\
\hline & $\mathrm{AcOH}$ & 1 & THF & 4 & 18 \\
\hline & $\mathrm{AcOH}$ & 1 & $\mathrm{MeOH}$ & 4 & 49 \\
\hline & $\mathrm{AcOH}$ & 1 & $\mathrm{H}_{2} \mathrm{O} / \mathrm{MeOH}(1: 10)$ & 4 & 32 \\
\hline & $\mathrm{B}(\mathrm{OH})_{3}$ & 1 & $\mathrm{H}_{2} \mathrm{O}$ & 12 & 16 \\
\hline & $\mathrm{B}(\mathrm{OH})_{3}$ & 1 & $\mathrm{MeOH}$ & 4 & 59 \\
\hline & $\mathrm{B}(\mathrm{OH})_{3}$ & 1 & $\mathrm{H}_{2} \mathrm{O} / \mathrm{MeOH}(1: 10)$ & 4 & 39 \\
\hline 10 & $\mathrm{~B}(\mathrm{OH})_{3}$ & 1 & $\mathrm{THF}$ & 4 & 41 \\
\hline 1 & $\mathrm{~B}(\mathrm{OH})_{3}$ & 1 & EtOAc & 4 & 0 \\
\hline 2 & $\mathrm{AcOH}$ & 1 & EtOAc & 4 & 0 \\
\hline 3 & $\mathrm{~B}(\mathrm{OMe})_{3}$ & 1 & none & 4 & 65 \\
\hline 4 & $\mathrm{~B}(\mathrm{OMe})_{3}$ & 1 & $\mathrm{MeOH}$ & 4 & 65 \\
\hline 16 & $\mathrm{~B}(\mathrm{OMe})_{3}$ & 2.0 & none & 4 & 68 \\
\hline 17 & $\mathrm{~B}(\mathrm{OMe})_{3}$ & 0.5 & none & 4 & 73 \\
\hline 18 & $\mathrm{~B}(\mathrm{OMe})_{3}$ & 0.2 & none & 4 & 71 \\
\hline 9 & $\mathrm{~B}(\mathrm{OMe})_{3}$ & 0.5 & none & 4 & $85^{c}$ \\
\hline
\end{tabular}

${ }^{a} 1: 1$ equivalent of 1a, 2a were used. Yields were calculated using ${ }^{1} \mathrm{H}$ NMR. ${ }^{b}$ From P. V. Ramachandran, P. V.; Gagare, P. D.; Sakavuyi, K.; Clark, P. Tetrahedron Lett., 2010, 51, 3167. ${ }^{c} 10 \%$ excess of amine was used. 


\section{NMR Data}

Dibenzyl amine (4aa) The product was isolated in $84 \%$ yield $(0.828 \mathrm{~g}, 4.2 \mathrm{mmol})$ as a colorless liquid after purification via column chromatography on silica gel using hexane/EtOAc (95:5) as an eluent. The spectral data matched with those reported in the literature. ${ }^{1}$

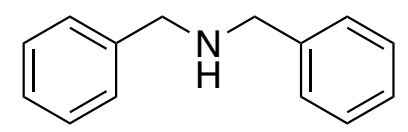

${ }^{1} \mathrm{H}$ NMR (300 MHz, Chloroform- $d$ ) $\delta 7.42-7.25$ (m, 10H), 3.85 (s, 4H), 2.09 (s, 1H).

${ }^{13} \mathrm{C}\{1 \mathrm{H}\} \mathrm{NMR}\left(75 \mathrm{MHz}, \mathrm{CDCl}_{3}\right) \delta 140.0,128.4,128.2,127.0,53.1$.

N-benzyl-1-(4-methoxyphenyl)methanamine (4ab) The product was isolated in 94\% yield (1.067 $\mathrm{g}, 4.7 \mathrm{mmol}$ ) as a pale yellow liquid after purification via column chromatography on silica gel using hexane/EtOAc (95:5) as an eluent. The spectral data matched with those reported in the literature. ${ }^{2}$<smiles>COc1ccc(CNCc2ccccc2)cc1</smiles>

${ }^{1} \mathrm{H}$ NMR $(300 \mathrm{MHz}$, Chloroform- $d$ ) $\delta 7.36-7.27(\mathrm{~m}, 8 \mathrm{H}), 6.89(\mathrm{~d}, J=8.6 \mathrm{~Hz}, 2 \mathrm{H}), 3.81(\mathrm{~s}, 4 \mathrm{H}), 3.76(\mathrm{~s}, 3 \mathrm{H})$ $2.29(\mathrm{~s}, 1 \mathrm{H})$.

${ }^{13} \mathrm{C}\{1 \mathrm{H}\} \mathrm{NMR}\left(75 \mathrm{MHz}, \mathrm{CDCl}_{3}\right) \delta 158.7,139.2,131.2,129.6,128.4,128.3,127.1,113.8,55.3,52.6,52.2$.

N-benzyl-1-(4-cholorophenyl)methanamine (4ac) The product was isolated in 96\% yield (1.108 g, $4.8 \mathrm{mmol}$ ) as a colorless liquid after purification via column chromatography on silica gel using hexane/EtOAc (95:5) as an eluent. The spectral data matched with those reported in the literature. ${ }^{2}$<smiles>Clc1ccc(CNCc2ccccc2)cc1</smiles>

${ }^{1} \mathrm{H}$ NMR (300 MHz, Chloroform- $d$ ) $\delta 7.37-7.25$ (m, 10H), 3.79 (dd, $\left.J=5.4,0.9 \mathrm{~Hz}, 4 \mathrm{H}\right), 1.90(\mathrm{~s}, 1 \mathrm{H})$.

${ }^{13} \mathrm{C}\{1 \mathrm{H}\}$ NMR $\left(75 \mathrm{MHz}, \mathrm{CDCl}_{3}\right) \delta 139.8,138.5,132.6,129.5,128.4,128.4,128.1,127.0,53.1,52.4$.

N-benzylaniline (4ad) The product was isolated in $85 \%$ yield $(0.777 \mathrm{~g}, 4.25 \mathrm{mmol})$ as a colorless liquid after purification via column chromatography on silica gel using hexane/EtOAc (95:5) as an eluent. The spectral data matched with those reported in the literature. ${ }^{2}$<smiles>c1ccc(CNc2ccccc2)cc1</smiles> 
N-benzyl-4-nitroaniline (4ag) The product was isolated in $64 \%$ yield $(0.729 \mathrm{~g}, 3.2 \mathrm{mmol})$ as an orange solid after purification via column chromatography on silica gel using hexane/EtOAc (9:1) as an eluent. The spectral data matched with those reported in the literature. ${ }^{2}$<smiles>O=[N+]([O-])c1ccc(NCc2ccccc2)cc1</smiles>

${ }^{1} \mathrm{H}$ NMR $(300 \mathrm{MHz}$, Chloroform- $d$ ) $\delta 8.13-8.06(\mathrm{~m}, 2 \mathrm{H}), 7.43-7.29(\mathrm{~m}, 5 \mathrm{H}), 6.63(\mathrm{~d}, J=9.1 \mathrm{~Hz}, 2 \mathrm{H}), 4.44$ (s, 2H).

${ }^{13} \mathrm{C}\{1 \mathrm{H}\} \mathrm{NMR}\left(75 \mathrm{MHz}, \mathrm{CDCl}_{3}\right) \delta 152.9,138.2,137.3,128.9,127.8,127.3,126.3,111.29,47.7$.

N-benzyl-4-methoxyaniline (4ae) The product was isolated in $84 \%$ yield $(0.894 \mathrm{~g}, 4.2 \mathrm{mmol})$ as a pale yellow solid after purification via column chromatography on silica gel using hexane/EtOAc (95:5) as an eluent. The spectral data matched with those reported in the literature. ${ }^{2}$<smiles>COc1ccc(NCc2ccccc2)cc1</smiles>

${ }^{1} \mathrm{H}$ NMR (300 MHz, Chloroform- $d$ ) $\delta 7.40-7.25$ (m, 5H), $6.83-6.75$ (m, 2H), $6.71-6.64$ (m, 2H), 4.29 (s, $2 \mathrm{H}), 3.75(\mathrm{~s}, 3 \mathrm{H})$.

${ }^{13} \mathrm{C}\{1 \mathrm{H}\} \mathrm{NMR}\left(75 \mathrm{MHz}, \mathrm{CDCl}_{3}\right) \delta 152.2,142.6,139.9,128.7,127.6,127.2,115.0,114.2,55.9,49.3$.

N-benzyl-2-methoxyaniline (4af) The product was isolated in 79\% yield $(0.841 \mathrm{~g}, 3.95 \mathrm{mmol})$ as a yellow solid after purification via column chromatography on silica gel using hexane/EtOAc (95:5) as an eluent. The spectral data matched with those reported in the literature. ${ }^{3}$<smiles>COc1ccccc1NCc1ccccc1</smiles>

${ }^{1} \mathrm{H}$ NMR (300 MHz, Chloroform- $d$ ) $\delta 7.42-7.26(\mathrm{~m}, 5 \mathrm{H}), 6.88-6.62(\mathrm{~m}, 4 \mathrm{H}), 4.37$ (s, 2H), $3.86(\mathrm{~s}, 3 \mathrm{H})$.

${ }^{13} \mathrm{C}\{1 \mathrm{H}\} \mathrm{NMR}\left(75 \mathrm{MHz}, \mathrm{CDCl}_{3}\right) \delta 146.7,139.4,128.5,127.5,127.1,121.2,116.7,110.2,109.3,55.5,48.1$

N-benzylpropan-1-amine (4ah) The product was isolated in $62 \%$ yield $(0.461 \mathrm{~g}, 3.1 \mathrm{mmol})$ as a yellow liquid after purification via column chromatography on silica gel using hexane/EtOAc (95:5) as an eluent. The spectral data matched with those reported in the literature. ${ }^{4}$<smiles>CCCNCc1ccccc1</smiles>

${ }^{1} \mathrm{H}$ NMR $(300 \mathrm{MHz}$, Chloroform- $d$ ) $\delta 7.50(\mathrm{~d}, J=6.6 \mathrm{~Hz}, 2 \mathrm{H}), 7.43-7.40(\mathrm{~m}, 2 \mathrm{H}), 7.36-7.32(\mathrm{~m}, 1 \mathrm{H}), 3.67$ (s, 2H), $2.68-2.34(\mathrm{~m}, 2 \mathrm{H}), 1.87-1.42(\mathrm{~m}, 2 \mathrm{H}), 0.98(\mathrm{t}, J=7.2 \mathrm{~Hz}, 3 \mathrm{H})$.

${ }^{13} \mathrm{C}\{1 \mathrm{H}\}$ NMR $\left(75 \mathrm{MHz}, \mathrm{CDCl}_{3}\right) \delta 140.1,128.8,128.2,126.7,58.5,55.6,20.4,12.1$. 
N-benzylpropan-2-amine (4ai) The product was isolated in $75 \%$ yield $(0.558 \mathrm{~g}, 3.75 \mathrm{mmol})$ as a yellow liquid after purification via column chromatography on silica gel using hexane as an eluent. The spectral data matched with those reported in the literature. ${ }^{5}$

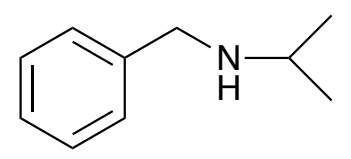

${ }^{1} \mathrm{H}$ NMR (300 MHz, Chloroform- $d$ ) $\delta 7.38-7.20(\mathrm{~m}, 5 \mathrm{H}), 3.79$ (s, 2H), $2.86(\mathrm{p}, J=6.2 \mathrm{~Hz}, 1 \mathrm{H}), 1.69(\mathrm{~s}, 1 \mathrm{H})$, $1.11(\mathrm{~d}, J=6.2 \mathrm{~Hz}, 6 \mathrm{H})$.

${ }^{13} \mathrm{C}\{1 \mathrm{H}\}$ NMR (75 MHz, $\left.\mathrm{CDCl}_{3}\right) \delta 134.9,129.3,128.6,128.0,49.3,48.1,20.6$.

Benzylpiperidine (4aj) The product was isolated in $71 \%$ yield $(0.621 \mathrm{~g}, 3.55 \mathrm{mmol})$ as a clear yellow liquid after purification via column chromatography on silica gel using hexane/EtOAc (95:5) as an eluent. The spectral data matched with those reported in the literature. ${ }^{6}$

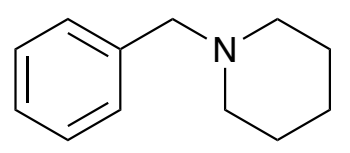

${ }^{1} \mathrm{H}$ NMR (300 MHz, Chloroform- $d$ ) $\delta 7.40-7.23(\mathrm{~m}, 5 \mathrm{H}), 3.51(\mathrm{~s}, 2 \mathrm{H}), 2.42(\mathrm{t}, J=5.3 \mathrm{~Hz}, 4 \mathrm{H}), 1.71-1.58$ (m, $4 \mathrm{H}), 1.48(\mathrm{td}, J=6.1,3.2 \mathrm{~Hz}, 2 \mathrm{H})$.

${ }^{13} \mathrm{C}\{1 \mathrm{H}\} \mathrm{NMR}\left(75 \mathrm{MHz}, \mathrm{CDCl}_{3}\right) \delta 138.6,129.2,128.0,126.8,64.0,54.6,26.2,24.6$.

(((4-chlorobenzyl)amino)methyl)phenol (4bc) The product was isolated in $77 \%$ yield $(0.950 \mathrm{~g}, 3.85$ $\mathrm{mmol})$ as a yellow oil after purification via column chromatography on silica gel using hexane/EtOAc (95:5) as an eluent. HRMS (CI) m/z: [M+H]+ calcd. for C14H15ClNO 248.0842, found 248.0840.<smiles>Oc1ccc(CNCc2ccc(Cl)cc2)cc1</smiles>

${ }^{1} \mathrm{H}$ NMR (300 MHz, Chloroform- $d$ ) $\delta 7.41-7.22(\mathrm{~m}, 4 \mathrm{H}), 7.22-7.03(\mathrm{~m}, 2 \mathrm{H}), 6.83-6.47(\mathrm{~m}, 2 \mathrm{H}), 3.74(\mathrm{~d}, J$ $=23.6 \mathrm{~Hz}, 4 \mathrm{H})$.

${ }^{13} \mathrm{C}\{1 \mathrm{H}\}$ NMR $\left(75 \mathrm{MHz}, \mathrm{CDCl}_{3}\right) \delta 156.0,138.6,132.1,130.3,129.4,129.2,128.2,115.3,52.4,52.0$.

N-(4-chlorobenzyl)-1-(4-nitrophenyl)methanamine (4cc) The product was isolated in 85\% yield $(1.176 \mathrm{~g}, 4.25 \mathrm{mmol})$ as a pale yellow solid after purification via column chromatography on silica gel using hexane/EtOAc (95:5) as an eluent. M.pt (92-93 C). HRMS (CI) m/z: [M+H]+ calcd. for C14H14ClN2O2 277.0744; found 277.0740.<smiles>O=[N+]([O-])c1ccc(CNCc2ccc(Cl)cc2)cc1</smiles>

${ }^{1} \mathrm{H}$ NMR (300 MHz, Chloroform- $d$ ) $\delta 8.26-8.10(\mathrm{~m}, 2 \mathrm{H}), 7.60-7.49$ (m, 2H), $7.40-7.18(\mathrm{~m}, 4 \mathrm{H}), 3.84(\mathrm{~d}, J$ $=33.7 \mathrm{~Hz}, 4 \mathrm{H}), 2.26(\mathrm{~s}, 1 \mathrm{H})$.

${ }^{13} \mathrm{C}\{1 \mathrm{H}\}$ NMR $\left(75 \mathrm{MHz}, \mathrm{CDCl}_{3}\right) \delta 152.0,142.3,139.5,128.5,127.4,127.1,114.8,114.0,55.8,49.3$. 
N-(4-chlorobenzyl)-1-cyclohexylmethanamine (4dc) The product was isolated in 87\% yield (1.030 $\mathrm{g}, 4.35 \mathrm{mmol}$ ) as a yellow oil after purification via column chromatography on silica gel using hexane/EtOAc (95:5) as an eluent. HRMS (CI) m/z: [M+H]+ calcd. for C14H21ClN 238.1362; found 238.1356.

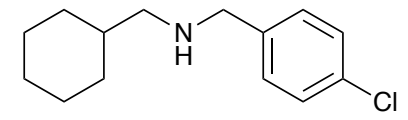

${ }^{1} \mathrm{H}$ NMR (300 MHz, Chloroform- $d$ ) $\delta 7.19$ (m, $\left.J=4.8 \mathrm{~Hz}, 4 \mathrm{H}\right), 3.65$ (d, $\left.J=5.2 \mathrm{~Hz}, 2 \mathrm{H}\right), 2.37$ (dd, $J=6.7,4.3$ $\mathrm{Hz}, 2 \mathrm{H}), 1.68(\mathrm{dt}, J=13.6,6.8 \mathrm{~Hz}, 4 \mathrm{H}), 1.41$ (ddd, $J=11.0,7.1,3.6 \mathrm{~Hz}, 1 \mathrm{H}), 1.16(\mathrm{dq}, J=14.3,10.9,10.5 \mathrm{~Hz}$, $4 \mathrm{H}), 1.00-0.67(\mathrm{~m}, 2 \mathrm{H})$.

${ }^{13} \mathrm{C}\{1 \mathrm{H}\} \operatorname{NMR}\left(75 \mathrm{MHz}, \mathrm{CDCl}_{3}\right) \delta 139.2,132.2,129.2,128.2,56.2,53.4,38.2,31.6,26.8,26.2$.

$\mathbf{N}$-(cyclohexylmethyl)aniline (4dd) The product was isolated in $78 \%$ yield $(0.621 \mathrm{~g}, 3.55 \mathrm{mmol})$ as a clear yellow liquid after purification via column chromatography on silica gel using hexane as an eluent. The spectral data matched with those reported in the literature. ${ }^{6}$<smiles>c1ccc(NCC2CCCCC2)cc1</smiles>

${ }^{1} \mathrm{H}$ NMR (300 MHz, Chloroform- $d$ ) $\delta 7.22-7.09$ (m, 2H), $6.76-6.63$ (m, 3H), $3.11(\mathrm{~s}, 2 \mathrm{H}), 1.71-1.45$ (m, $9 \mathrm{H}), 1.40-1.26(\mathrm{~m}, 2 \mathrm{H})$.

${ }^{13} \mathrm{C}\{1 \mathrm{H}\} \mathrm{NMR}\left(75 \mathrm{MHz}, \mathrm{CDCl}_{3}\right) \delta 148.7,129.2,117.6,113.2,71.5,54.3,35.9,26.0,22.1$.

N-benzyl-1-cyclohexylmethanamine (4da) The product was isolated in 83\% yield $(0.791 \mathrm{~g}, 3.9$ mmol) as a pale yellow liquid after purification via column chromatography on silica gel using hexane as an eluent. The spectral data matched with those reported in the literature. ${ }^{7}$<smiles>c1ccc(CNCC2CCCCC2)cc1</smiles>

${ }^{1} \mathrm{H}$ NMR (300 MHz, Chloroform- $d$ ) $\delta 7.31$ (dd, $\left.J=23.7,4.5 \mathrm{~Hz}, 5 \mathrm{H}\right), 3.80$ (s, $\left.2 \mathrm{H}\right), 2.50(\mathrm{~d}, J=6.7 \mathrm{~Hz}, 2 \mathrm{H}$ ), $1.89-1.63(\mathrm{~m}, 4 \mathrm{H}), 1.62-1.46(\mathrm{~m}, 1 \mathrm{H}), 1.42-1.09(\mathrm{~m}, 4 \mathrm{H}), 0.97$ (td, $J=11.7,2.9 \mathrm{~Hz}, 2 \mathrm{H})$.

${ }^{13} \mathrm{C}\{1 \mathrm{H}\}$ NMR $\left(75 \mathrm{MHz}, \mathrm{CDCl}_{3}\right) \delta 140.6,128.3,128.1,128.0,126.8,56.3,54.3,38.1,31.9,31.6,26.9,26.3$.

N-(4-methoxybenzyl)-1-(1-methyl-1H-pyrrol-2-yl)methanamine (4eb) ): The product was isolated in $97 \%$ yield ( $1.115 \mathrm{~g}, 4.85 \mathrm{mmol}$ ) as a dark brown liquid after purification via column chromatography on silica gel using hexane/EtOAc (95:5) as an eluent. HRMS (CI) m/z: $[\mathrm{M}+\mathrm{H}]+$ calcd. for $\mathrm{C}_{14} \mathrm{H}_{19} \mathrm{~N}_{2} \mathrm{O} 231.1497$; found 231.1493 .<smiles>COc1ccc(CNCc2cccn2C)cc1</smiles>

${ }^{1} \mathrm{H}$ NMR $(300 \mathrm{MHz}$, Chloroform- $d$ ) $\delta 7.28(\mathrm{~d}, J=8.4 \mathrm{~Hz}, 2 \mathrm{H}), 6.89(\mathrm{~d}, J=8.5 \mathrm{~Hz}, 2 \mathrm{H}), 6.60(\mathrm{t}, J=2.1 \mathrm{~Hz}, 1 \mathrm{H})$, $6.06(\mathrm{~d}, J=2.6 \mathrm{~Hz}, 2 \mathrm{H}), 3.89-3.70(\mathrm{~m}, 7 \mathrm{H}), 3.64(\mathrm{~s}, 3 \mathrm{H}), 1.94(\mathrm{~s}, 1 \mathrm{H})$.

${ }^{13} \mathrm{C}\{1 \mathrm{H}\} \mathrm{NMR}\left(75 \mathrm{MHz}, \mathrm{CDCl}_{3}\right) \delta 158.6,132.6,131.1,129.3,122.4,113.8,108.0,106.5,1,55.3,52.8,44.9$. 
N-(4-chlorobenzyl)-1-(furan-2-yl)methanamine (4fe) The product was isolated in $90 \%$ yield $(0.994 \mathrm{~g}, 4.50 \mathrm{mmol})$ as a brown oil after purification via column chromatography on silica gel using hexane/EtOAc (95:5) as an eluent. HRMS (CI) m/z: [M+H]+ calcd. for $\mathrm{C}_{12} \mathrm{H}_{13} \mathrm{ClNO} 222.0685$; found 222.0682.<smiles>Clc1ccc(CNCc2ccco2)cc1</smiles>

${ }^{1} \mathrm{H}$ NMR $(300 \mathrm{MHz}$, Chloroform- $d$ ) $\delta 7.50-7.05(\mathrm{~m}, 5 \mathrm{H}), 6.31(\mathrm{dd}, J=3.2,1.9 \mathrm{~Hz}, 1 \mathrm{H}), 6.17(\mathrm{~d}, J=3.2 \mathrm{~Hz}$, $1 \mathrm{H}), 3.74(\mathrm{~d}, J=6.5 \mathrm{~Hz}, 4 \mathrm{H}), 1.98-1.70(\mathrm{~m}, 1 \mathrm{H})$.

${ }^{13} \mathrm{C}\{1 \mathrm{H}\}$ NMR $\left(75 \mathrm{MHz}, \mathrm{CDCl}_{3}\right) \delta 153.4,141.8,138.2,132.6,129.5,129.5,110.1,107.2,52.0,45.3$.

N-(4-chlorobenzyl)cyclohexanamine (4gc) The product was isolated in $80 \%$ yield ( $0.892 \mathrm{~g}, 4.00$ $\mathrm{mmol}$ ) as a yellow liquid after purification via column chromatography on silica gel using hexane as an eluent. HRMS (CI) m/z: [M+H]+ calcd. for C13H19ClN 224.1206; found 224.1203.<smiles>Clc1ccc(CNC2CCCCC2)cc1</smiles>

${ }^{1} \mathrm{H}$ NMR (300 MHz, Chloroform- $d$ ) $\delta 7.52-7.09$ (m, 4H), 3.77 (s, 2H), 2.45 (tt, $\left.J=10.1,3.7 \mathrm{~Hz}, 1 \mathrm{H}\right), 1.89$ (dd, $J=11.8,3.8 \mathrm{~Hz}, 2 \mathrm{H}), 1.81-1.69(\mathrm{~m}, 2 \mathrm{H}), 1.66-1.54(\mathrm{~m}, 1 \mathrm{H}), 1.38-0.87$ (m, $5 \mathrm{H})$.

${ }^{13} \mathrm{C}\{1 \mathrm{H}\}$ NMR $\left(75 \mathrm{MHz}, \mathrm{CDCl}_{3}\right) \delta 139.4,132.3,129.3,128.3,56.1,50.3,33.6,26.2,25.1$.

N-benzylcyclopentanamine (4ha) The product was isolated in $75 \%$ yield $(0.791 \mathrm{~g}, 3.9 \mathrm{mmol})$ as a yellow oil after purification via column chromatography on silica gel using hexane/EtOAc (9:1) as an eluent. The spectral data matched with those reported in the literature. ${ }^{8}$

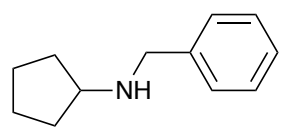

${ }^{1} \mathrm{H}$ NMR (300 MHz, Chloroform- $d$ ) $\delta 7.25(\mathrm{~d}, J=8.9 \mathrm{~Hz}, 5 \mathrm{H}), 3.72(\mathrm{~d}, J=9.4 \mathrm{~Hz}, 2 \mathrm{H}), 3.06$ (p, $J=6.6 \mathrm{~Hz}$, $1 \mathrm{H}), 1.95-1.20(\mathrm{~m}, 8 \mathrm{H})$.

${ }^{13} \mathrm{C}\{1 \mathrm{H}\}$ NMR $\left(75 \mathrm{MHz}, \mathrm{CDCl}_{3}\right) \delta 140.3,128.3,128.2,126.8,59.1,52.7,33.2,24.2$.

N-(4-methoxybenzyl)pentan-3-amine (4ib) The product was isolated in 73\% yield ( $0.755 \mathrm{~g}, 3.65 \mathrm{mmol})$ as a yellow liquid after purification via column chromatography on silica gel using hexane/EtOAc (9:1) as an eluent. HRMS (CI) m/z: [M+H]+ calcd. for $\mathrm{C}_{13} \mathrm{H}_{22} \mathrm{NO} 208.1696$; found 208.1701.

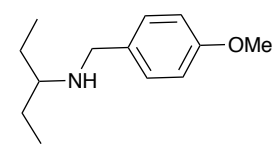

${ }^{1} \mathrm{H}$ NMR (300 MHz, Chloroform- $d$ ) $\delta 7.34-7.17$ (m, 2H), $6.91-6.73(\mathrm{~m}, 2 \mathrm{H}), 3.79$ (s, 3H), 3.69 (s, 2H), 2.43 (p, $J=5.9 \mathrm{~Hz}, 1 \mathrm{H}), 1.46$ (qdd, $J=7.3,5.9,1.8 \mathrm{~Hz}, 4 \mathrm{H}), 1.25(\mathrm{~d}, J=13.0 \mathrm{~Hz}, 1 \mathrm{H}), 0.89(\mathrm{t}, J=7.5 \mathrm{~Hz}, 6 \mathrm{H})$.

${ }^{13} \mathrm{C}\{1 \mathrm{H}\}$ NMR $\left(75 \mathrm{MHz}, \mathrm{CDCl}_{3}\right) \delta 158.4,133.2,113.6,59.2,55.2,50.5,25.7,9.8$. 
N-(4-methoxybenzyl)-1-phenylethan-1-amine (4jb) The product was isolated in 85\% yield (1.024 $\mathrm{g}, 4.25 \mathrm{mmol}$ ) as a light yellow liquid after purification via column chromatography on silica gel using hexane/EtOAc (95:5) as an eluent. HRMS (CI) m/z: [M+H]+ calcd. for $\mathrm{C}_{16} \mathrm{H}_{20} \mathrm{NO} 242.1545$; found 242.1541 .<smiles>COc1ccc(CNC(c2ccccc2)[N+](=O)[O-])cc1</smiles>

${ }^{1} \mathrm{H}$ NMR $(300 \mathrm{MHz}$, Chloroform- $d$ ) $\delta 7.53-7.07(\mathrm{~m}, 7 \mathrm{H}), 7.01-6.75(\mathrm{~m}, 2 \mathrm{H}), 3.82(\mathrm{~d}, J=8.1 \mathrm{~Hz}, 5 \mathrm{H}), 3.59$ $(\mathrm{d}, J=8.2 \mathrm{~Hz}, 1 \mathrm{H}), 1.61(\mathrm{~s}, 1 \mathrm{H}), 1.39(\mathrm{~d}, J=6.6 \mathrm{~Hz}, 3 \mathrm{H})$.

${ }^{13} \mathrm{C}\{1 \mathrm{H}\} \mathrm{NMR}\left(75 \mathrm{MHz}, \mathrm{CDCl}_{3}\right) \delta 158.4,145.5,132.7,129.2,128.4,126.6,113.7,57.4,55.3,51.1,24.6$.

N-(4-methoxybenzyl)-2,3-dihydro-1H-inden-1-amine (4kb) The product was isolated in 98\% yield $(1.239 \mathrm{~g}, 4.90 \mathrm{mmol})$ as a dark brown liquid after purification via column chromatography on silica gel using hexane/EtOAc (95:5) as an eluent. HRMS (CI) m/z: [M+H]+ calcd. for $\mathrm{C}_{17} \mathrm{H}_{20} \mathrm{NO} 254.1545$; found 254.1544.

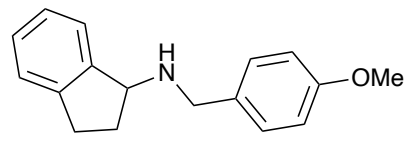

${ }^{1} \mathrm{H}$ NMR (300 MHz, Chloroform- $d$ ) $\delta 7.49-7.13(\mathrm{~m}, 6 \mathrm{H}), 6.87(\mathrm{~d}, J=8.7 \mathrm{~Hz}, 2 \mathrm{H}), 4.30(\mathrm{t}, J=6.5 \mathrm{~Hz}, 1 \mathrm{H})$, $3.81(\mathrm{~d}, J=15.4 \mathrm{~Hz}, 5 \mathrm{H}), 3.12-2.95(\mathrm{~m}, 1 \mathrm{H}), 2.82(\mathrm{dt}, J=15.8,7.6 \mathrm{~Hz}, 1 \mathrm{H}), 2.52-2.32(\mathrm{~m}, 1 \mathrm{H}), 2.03-1.82$ $(\mathrm{m}, 1 \mathrm{H})$.

${ }^{13} \mathrm{C}\{1 \mathrm{H}\} \mathrm{NMR}\left(75 \mathrm{MHz}, \mathrm{CDCl}_{3}\right) \delta 158.4,145.2,143.5,132.6,129.2,127.3,126.1,124.6,124.0,113.7,62.6$,

$55.3,50.8,33.7,30.5$.

N-allyl-2,3-dihydro-1H-inden-1-amine (4kk) The product was isolated in 93\% yield (0.804 g, 4.65 $\mathrm{mmol}$ ) as a brown oil after purification via column chromatography on silica gel using hexane/EtOAc (95:5) as an eluent. HRMS (CI) m/z: [M+H]+ calcd. for $\mathrm{C}_{12} \mathrm{H}_{16} \mathrm{~N}$ 174.1282; found 174.1280<smiles>C=CCNC1CCc2ccccc21</smiles>

${ }^{1} \mathrm{H}$ NMR $(300 \mathrm{MHz}$, Chloroform- $d$ ) $\delta 7.45-7.33(\mathrm{~m}, 1 \mathrm{H}), 7.22(\mathrm{dd}, J=8.6,3.1 \mathrm{~Hz}, 3 \mathrm{H}), 6.09-5.92(\mathrm{~m}, 1 \mathrm{H})$, $5.31-5.09(\mathrm{~m}, 2 \mathrm{H}), 4.32-4.25(\mathrm{~m}, 1 \mathrm{H}), 3.39$ (ddd, $J=6.1,3.3,1.5 \mathrm{~Hz}, 2 \mathrm{H}), 3.03(\mathrm{ddd}, J=13.6,8.5,5.0 \mathrm{~Hz}$, $1 \mathrm{H}), 2.83(\mathrm{dt}, J=15.6,7.6 \mathrm{~Hz}, 1 \mathrm{H}), 2.43$ (dddd, $J=12.0,8.2,6.5,5.0 \mathrm{~Hz}, 1 \mathrm{H}), 1.74(\mathrm{~m}, 2 \mathrm{H})$.

${ }^{13} \mathrm{C}\{1 \mathrm{H}\} \mathrm{NMR}\left(75 \mathrm{MHz}, \mathrm{CDCl}_{3}\right) \delta 145.0,143.6,136.9,127.4,126.2,124.7,124.1,115.9,62.7,50.0,33.7$, 30.5 .

Rasagiline (4kl) The product was isolated in $92 \%$ yield $(0.786 \mathrm{~g}, 4.6 \mathrm{mmol})$ as a solid, $\mathrm{mp}=148^{\circ} \mathrm{C}$, after purification via column chromatography on silica gel using hexane/EtOAc (95:5) as an eluent. The spectral data matched with those reported in the literature. ${ }^{9}$

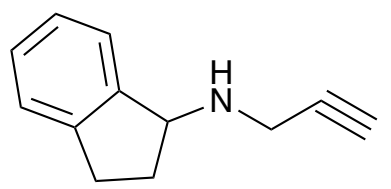

${ }^{1} \mathrm{H}$ NMR (300 MHz, Chloroform- $d$ ) $\delta 7.47-7.10$ (m, 4H), $4.43(\mathrm{dd}, J=6.8,5.3 \mathrm{~Hz}, 1 \mathrm{H}), 3.53(\mathrm{dd}, J=2.5,1.5$ Hz, 2H), 3.07 (ddd, $J=16.1,8.3,5.7 \mathrm{~Hz}, 1 \mathrm{H}$ ), 2.84 (ddd, $J=15.3,8.3,6.2 \mathrm{~Hz}, 1 \mathrm{H}$ ), 2.41 (ddddd, $J=12.6,8.0$, 6.8, $5.7,1.0 \mathrm{~Hz}, 2 \mathrm{H}), 1.97-1.78(\mathrm{~m}, 1 \mathrm{H})$.

${ }^{13} \mathrm{C}\{1 \mathrm{H}\} \mathrm{NMR}\left(75 \mathrm{MHz}, \mathrm{CDCl}_{3}\right) \delta 144.4,143.7,127.5,126.2,124.8,124.2,82.6,71.6,61.9,36.2,33.5,30.6$. 


\section{REFERENCES}

(1) Anikin, O. V.; Russian Chem. Bull., 2009, 58, 2043-2046.

(2) Likhar, P.R., Arundhathi, R., Kantam, M.L., Prathima, P.S., Eur. J. Org. Chem.2009, 5383-5389.

(3) Cano, R.; RamóN, D. J.; Yus, M., J.Org.Chem., 2011, 76, 5547-5557.

(4) Tajbakhsh, M.; Lakouraj, M.M.; Mahalli, M.S.. Lett. Org. Chem., 2008, 5, 148-152.

(5) García Ruano, J. L.; Parra, A.; Alemán, J.; Yuste, F.; Mastranzo, V. M.. Chem. Commun. 2009, 404406.

(6) Molander, G. A.; Gormisky, P. E.; Sandrock, D. L.. J.Org.Chem 2008, 73, 2052-2057

(7) Byun, E.; Hong, B.; De Castro, K. A.; Lim, M.; Rhee, H.. J. Org. Chem. 2007, 72, 9815-9817.

(8) Zhang, W.; Dong, X.; Zhao, W. Org. Lett. 2011, 14, 5386-5389.

(9) Reddy, R. P.; Davies, H. M. L. Org. Lett. 2006, 9, 5013-5016. 


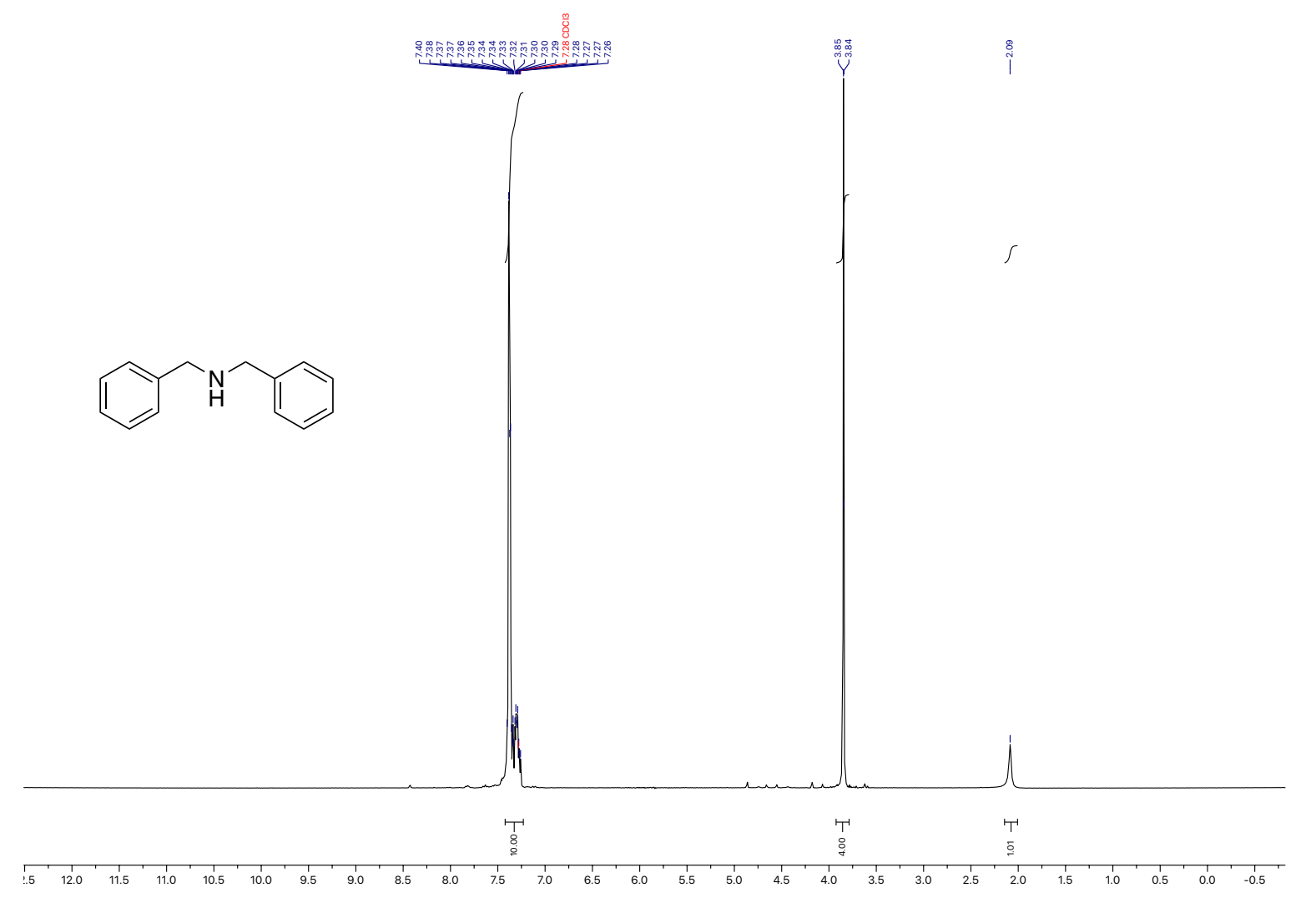

$\underline{300 \mathrm{MHz}}{ }^{1} \mathrm{H}$ NMR of Dibenzylamine (4aa) in $\mathrm{CDCl}_{3}$<smiles>c1ccc(CNCc2ccccc2)cc1</smiles>

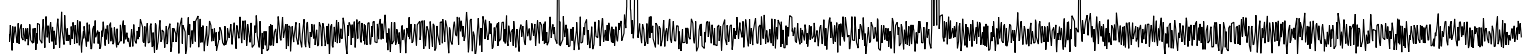

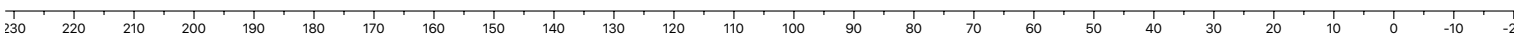

$\underline{75 \mathrm{MHz}}{ }^{13} \mathrm{C}\{\mathrm{H}\}$ NMR of Dibenzylamine (4aa) in $\mathrm{CDCl}_{3}$ 


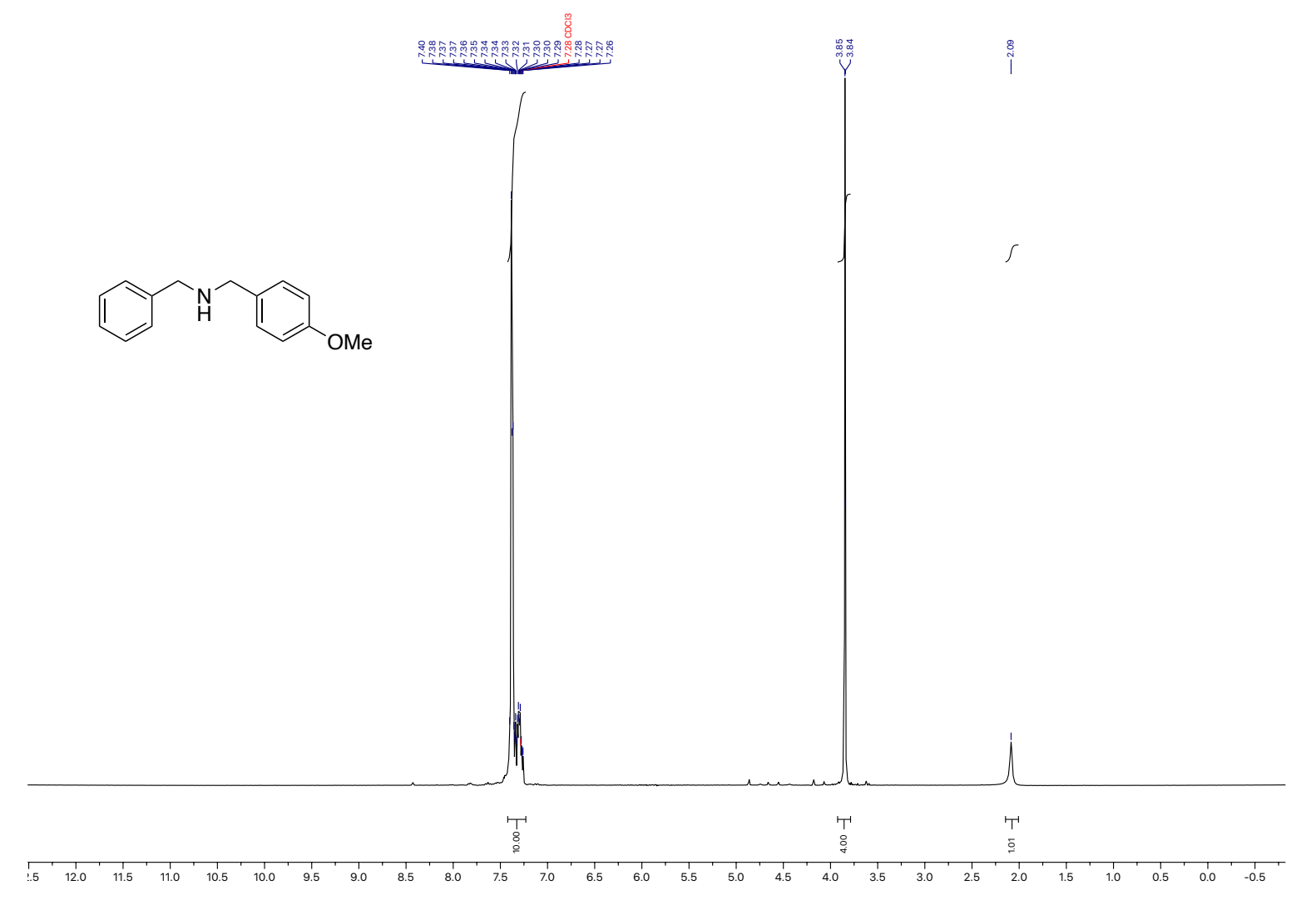

$\underline{300 ~ M H z}{ }^{1} \mathrm{H}$ NMR of N-benzyl-1-(4-methoxyphenyl)methanamine (4ab) in $\mathrm{CDCl}_{3}$,
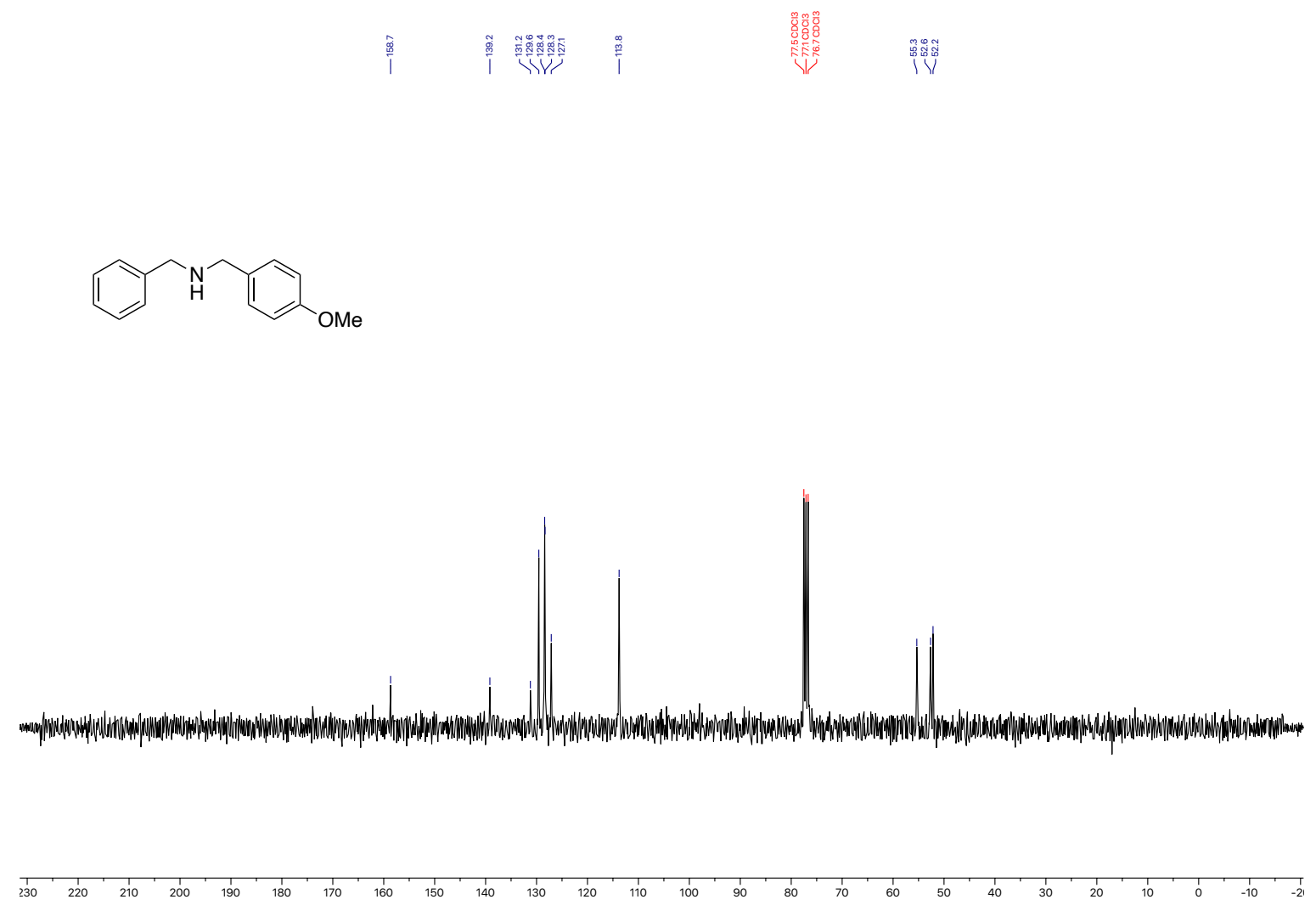

$75 \mathrm{MHz}{ }^{13} \mathrm{C}\{\mathrm{H}\}$ NMR of N-benzyl-1-(4-methoxyphenyl)methanamine (4ab) in $\mathrm{CDCl}_{3}$ 


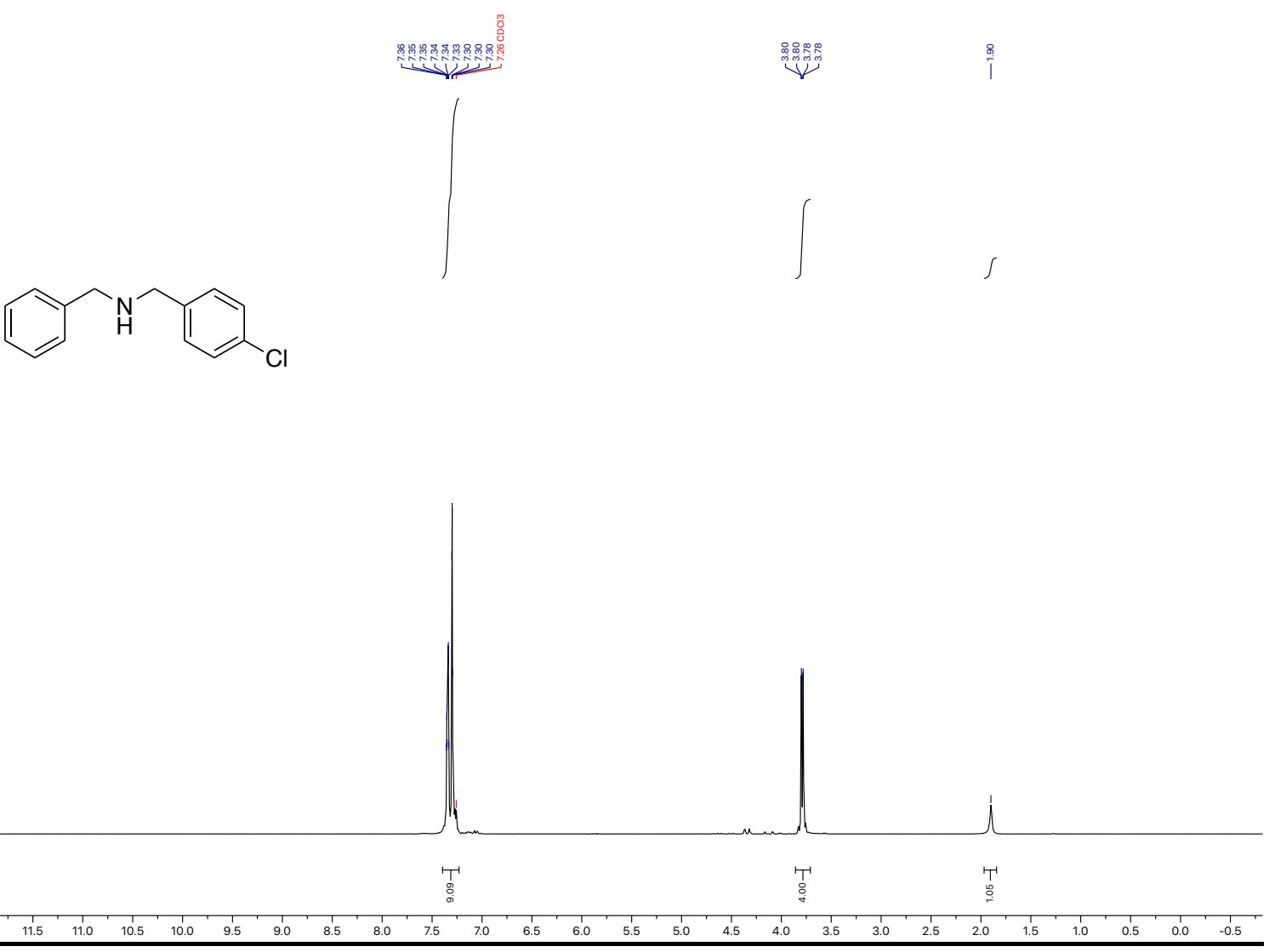

$\underline{300 ~ M H z}{ }^{1} \mathrm{H}$ NMR of N-benzyl-1-(4-chlorophenyl)methanamine (4ac) in $\mathrm{CDCl}_{3}$<smiles>Clc1ccc(CNCc2ccccc2)cc1</smiles>
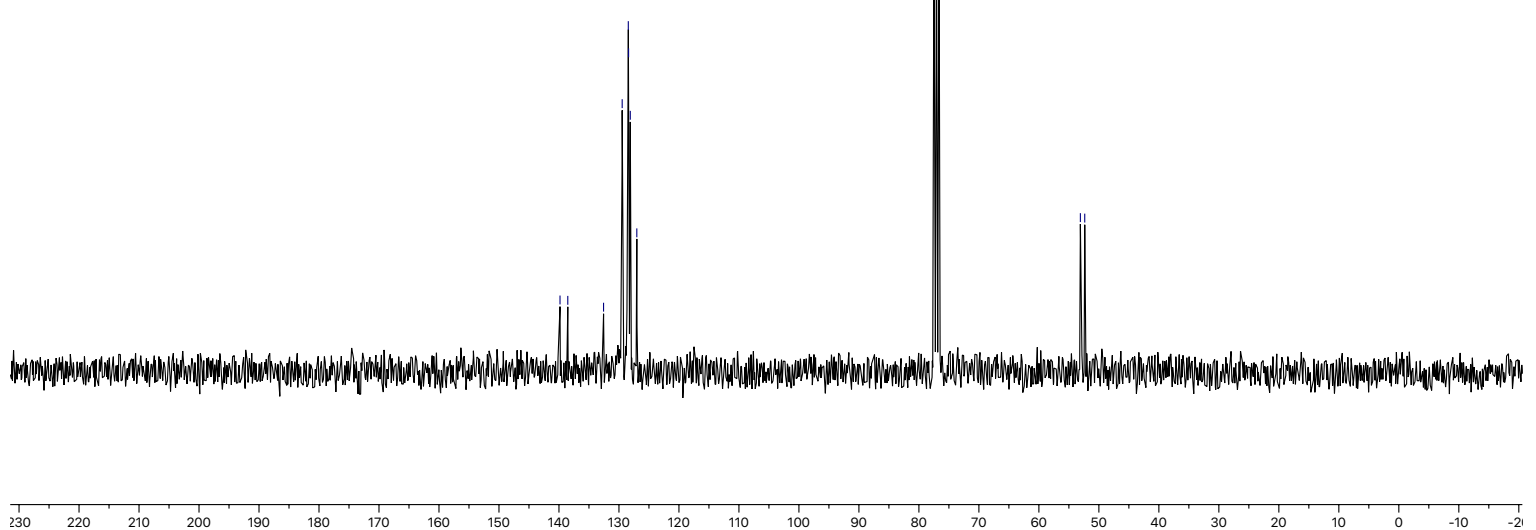

$\underline{75 \mathrm{MHz}}{ }^{13} \mathrm{C}\{\mathrm{H}\}$ NMR of N-benzyl-1-(4-chlorophenyl)methanamine (4ac) in $\mathrm{CDCl}_{3}$ 

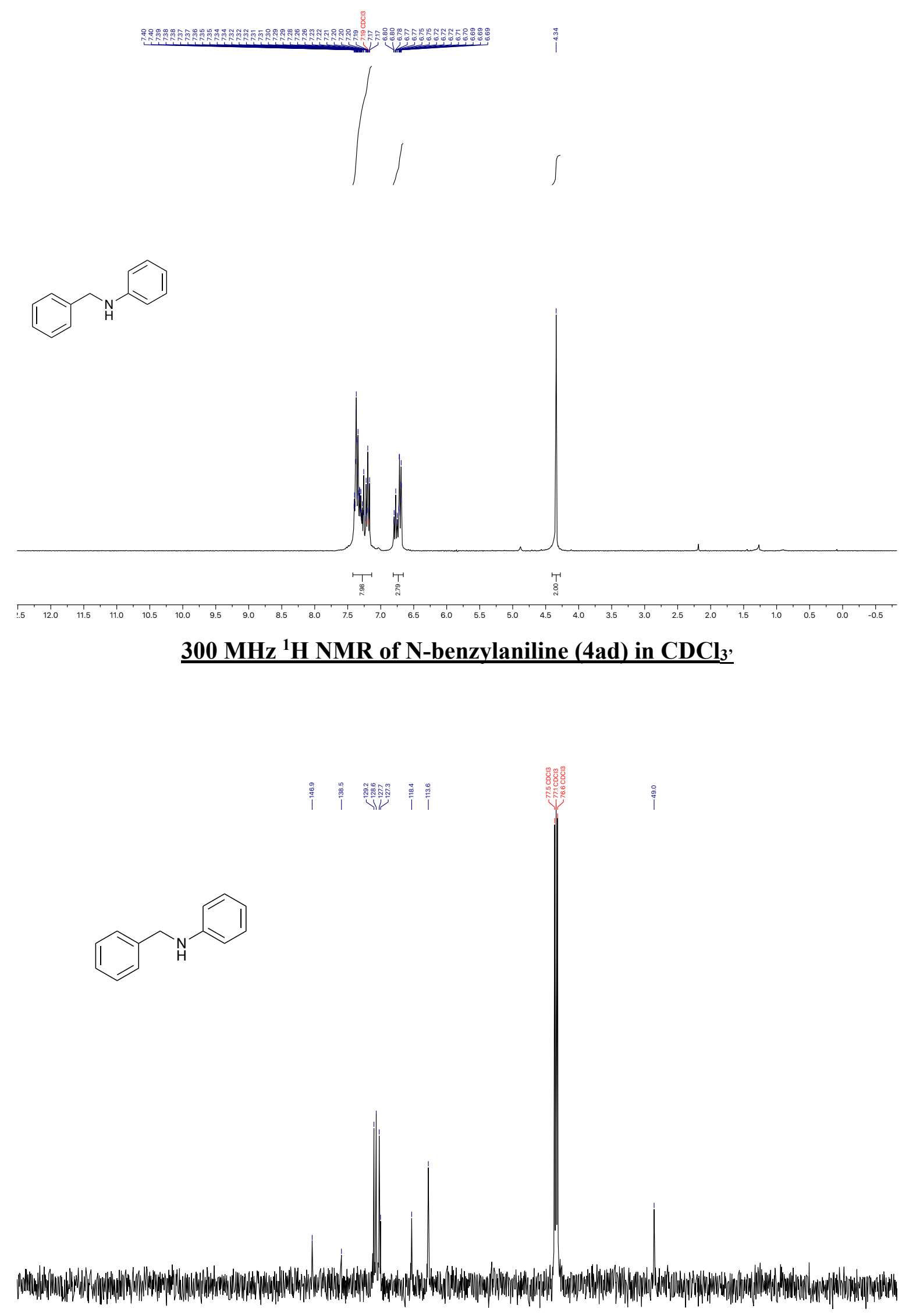

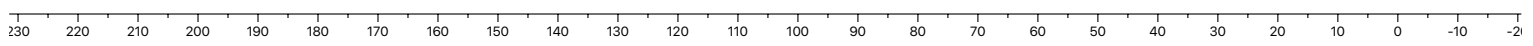



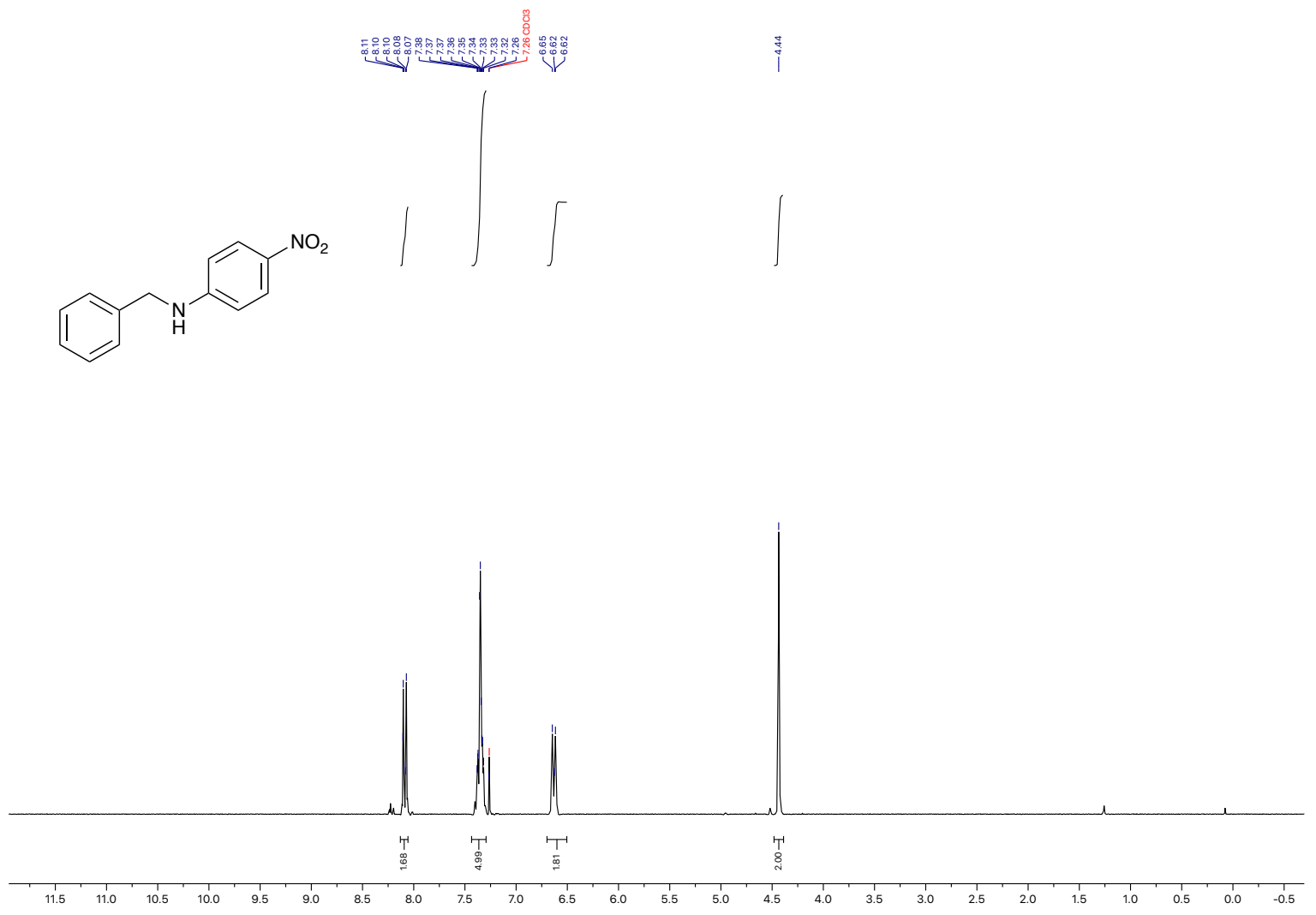

$\underline{300 \mathrm{MHz}}{ }^{1} \mathrm{H}$ NMR of $\mathrm{N}-$ benzyl-4-nitroaniline (4ag) in $\mathrm{CDCl}_{3}$
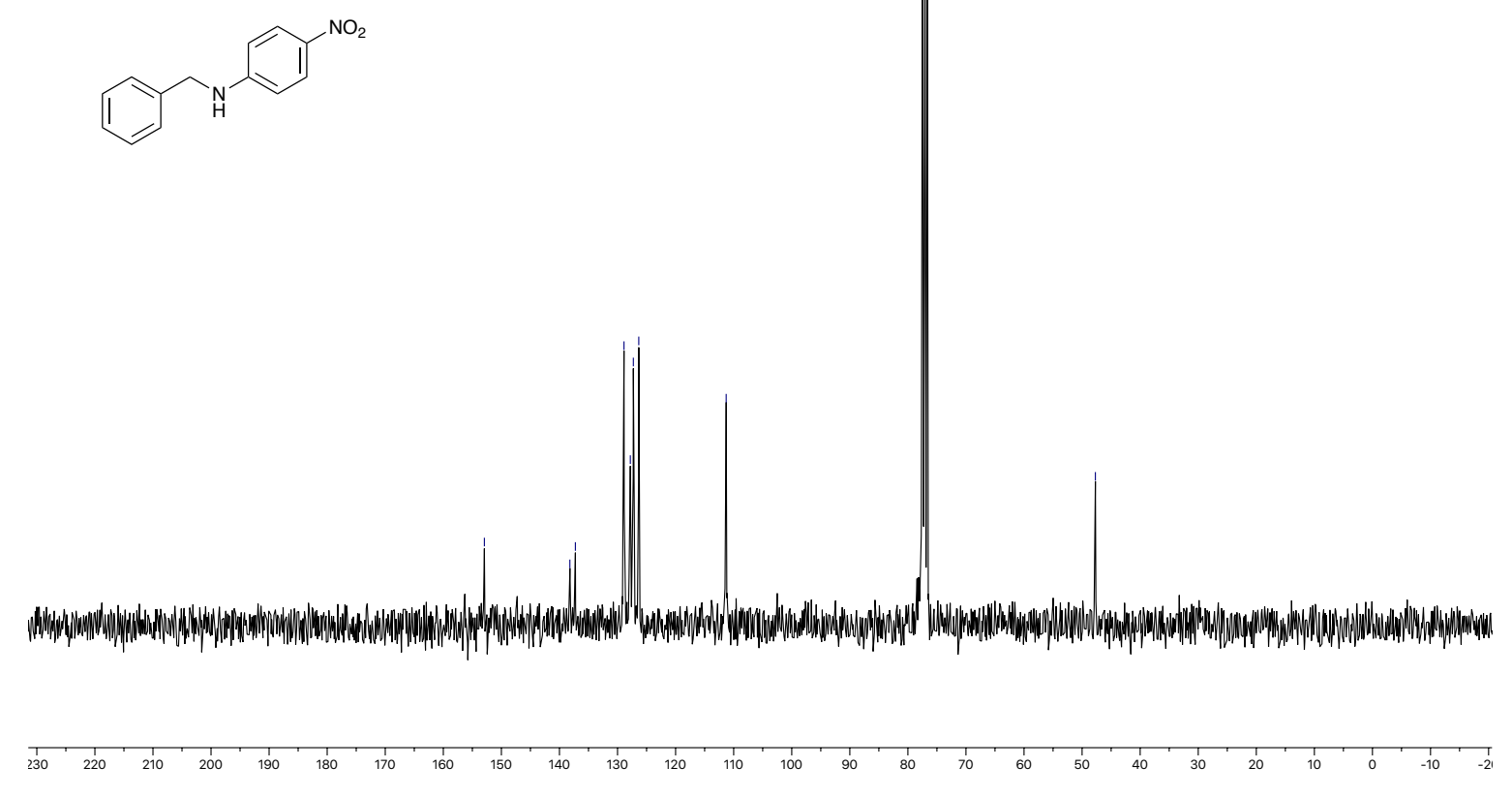

$\underline{75 \mathrm{MHz}}{ }^{13} \mathrm{C}\{\mathrm{H}\}$ NMR of $\mathrm{N}$-benzyl-4-nitroaniline (4ag) in $\mathrm{CDCl}_{3}$ 


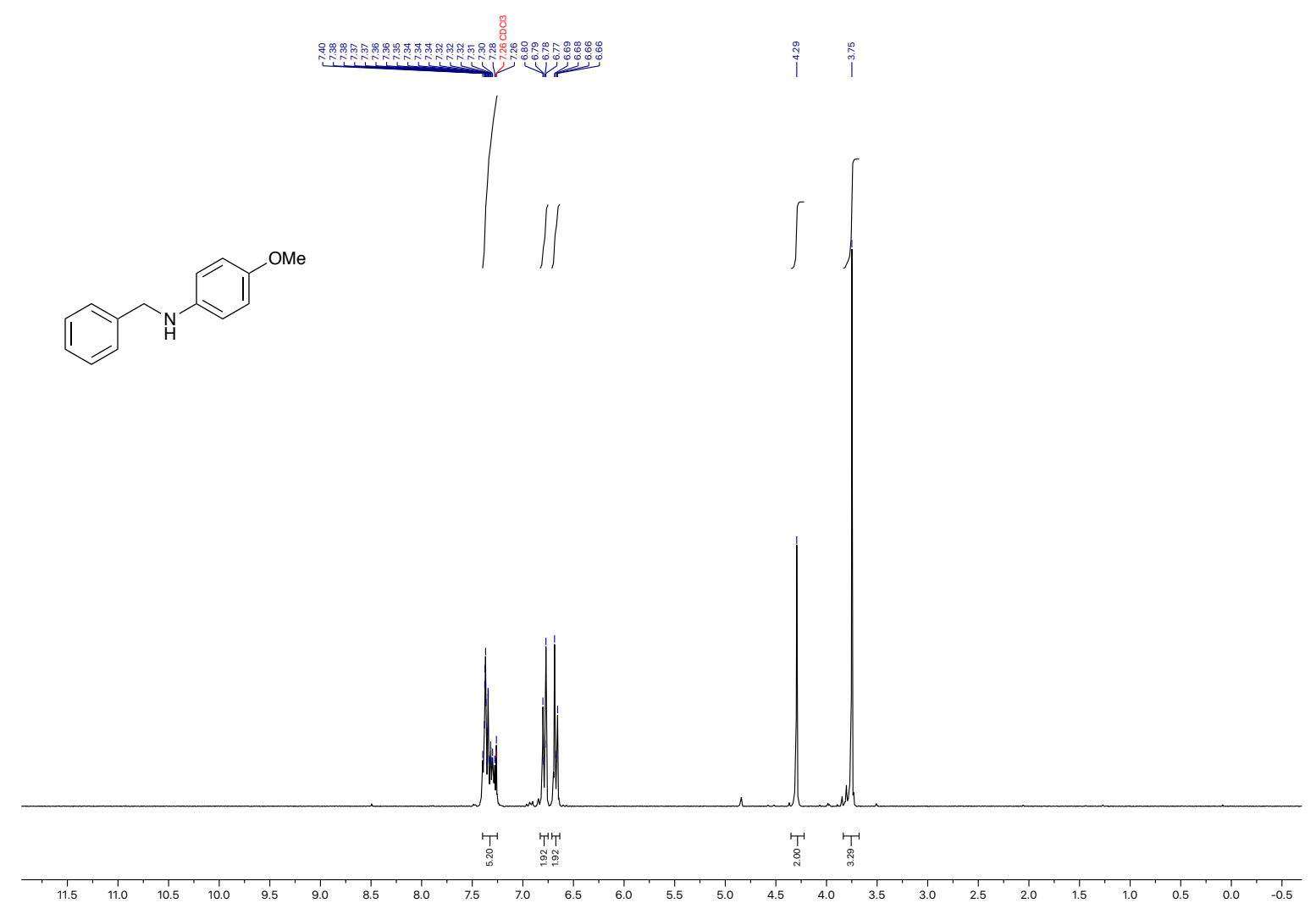

$\underline{300 \mathrm{MHz}}{ }^{1} \mathrm{H}$ NMR of $\mathrm{N}$-benzyl-4-methoxyaniline (4ae) in $\underline{\mathrm{CDCl}_{3}}$

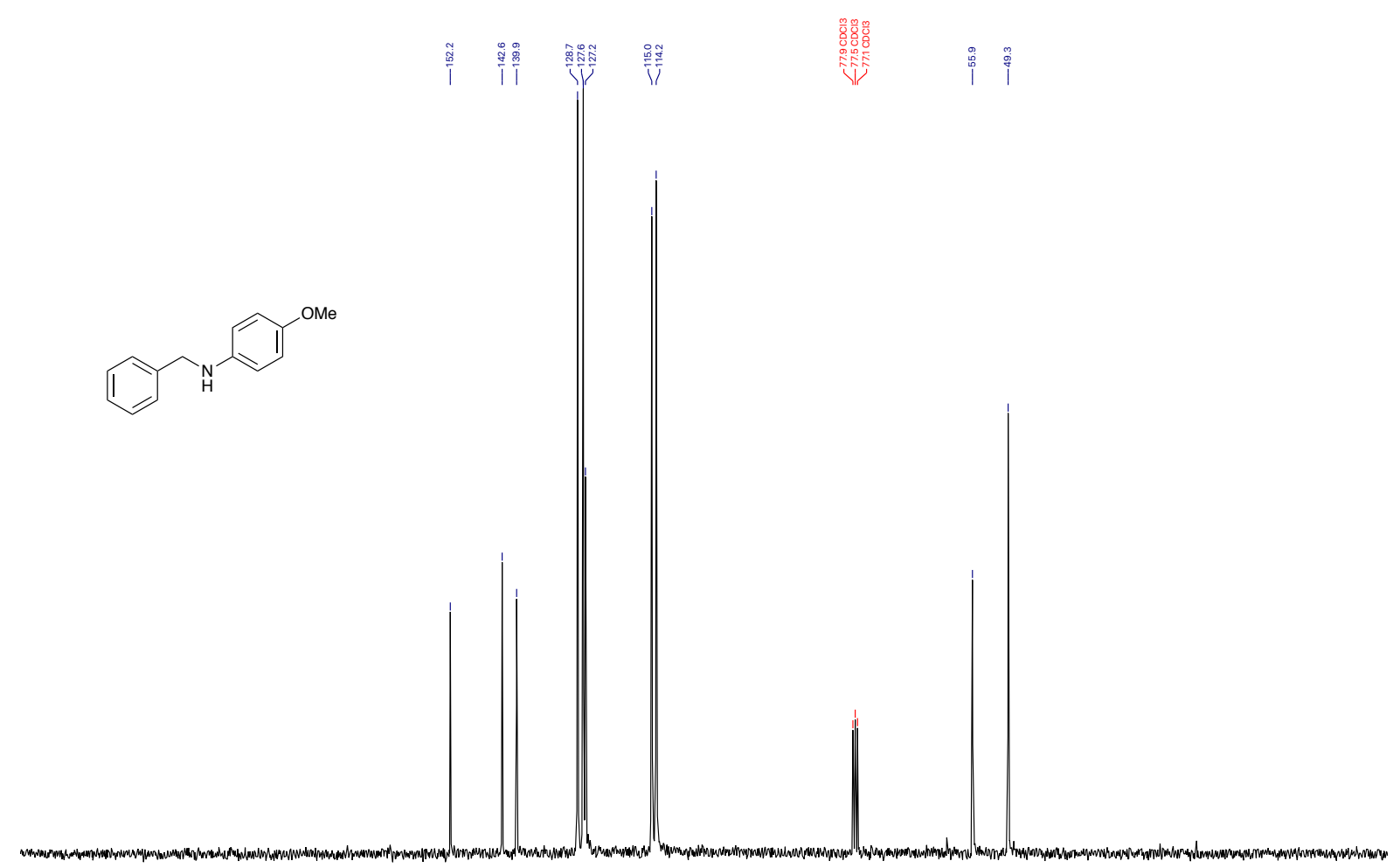

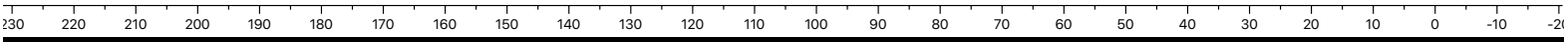




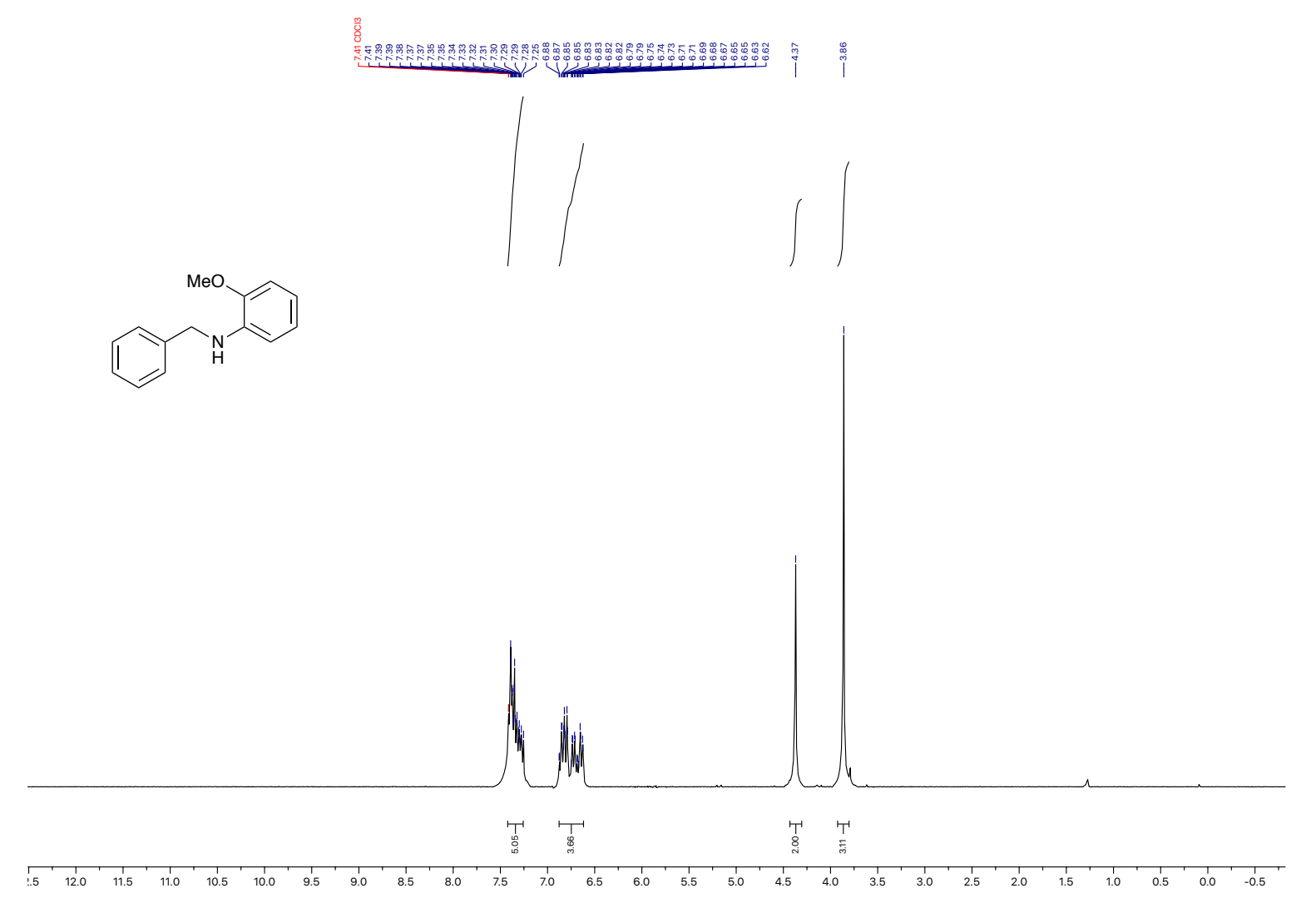

$\underline{300 \mathrm{MHz}}{ }^{1} \mathrm{H}$ NMR of $\mathrm{N}-$ benzyl-2-methoxyaniline (4af) in $\underline{\mathrm{CDCl}_{3}}$
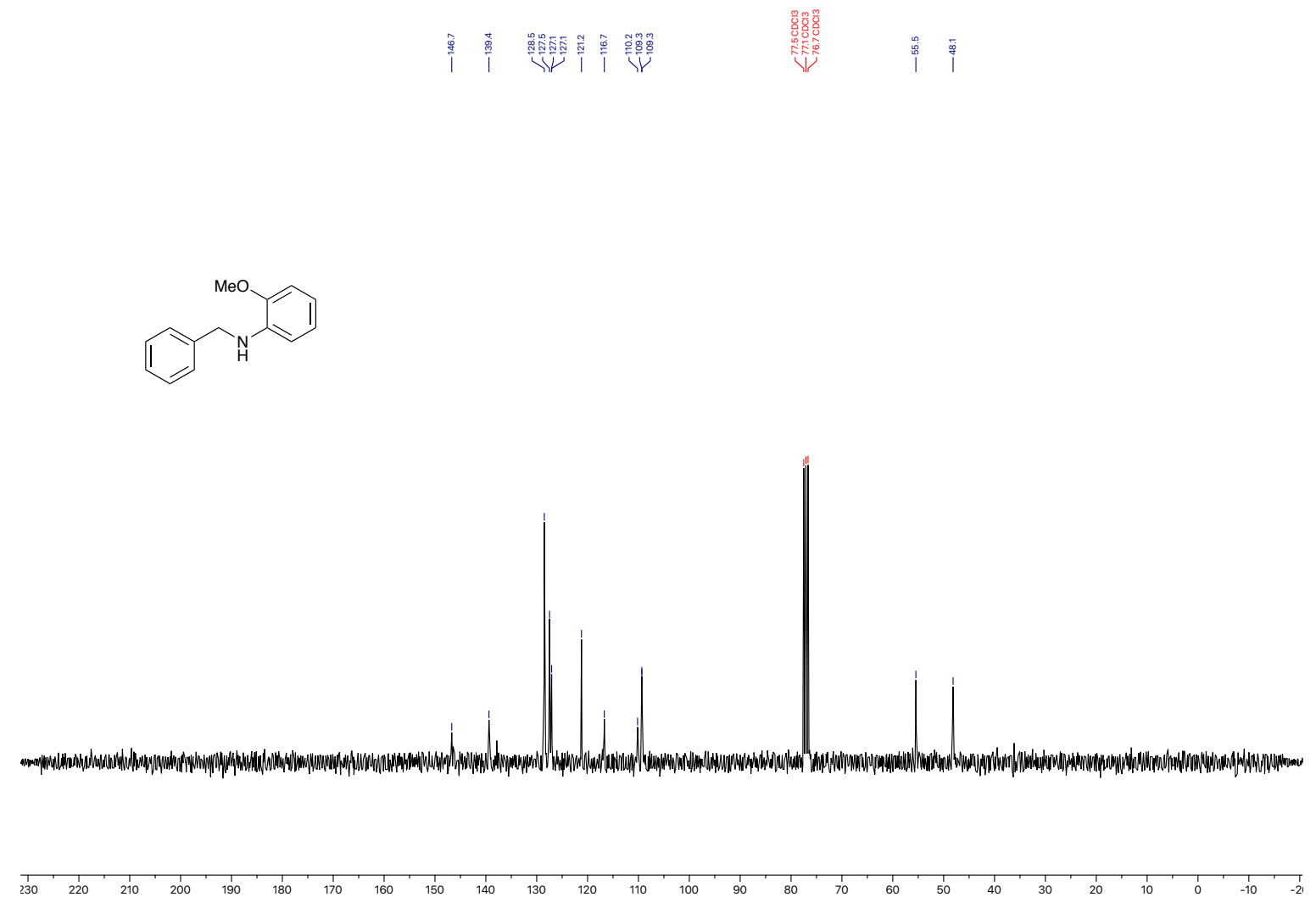

$\underline{75 \mathrm{MHz}}{ }^{13} \mathrm{C}\{\mathrm{H}\}$ NMR of $\mathrm{N}-$ benzyl-2-methoxyaniline (4af) in $\underline{\mathrm{CDCl}_{3}}$ 


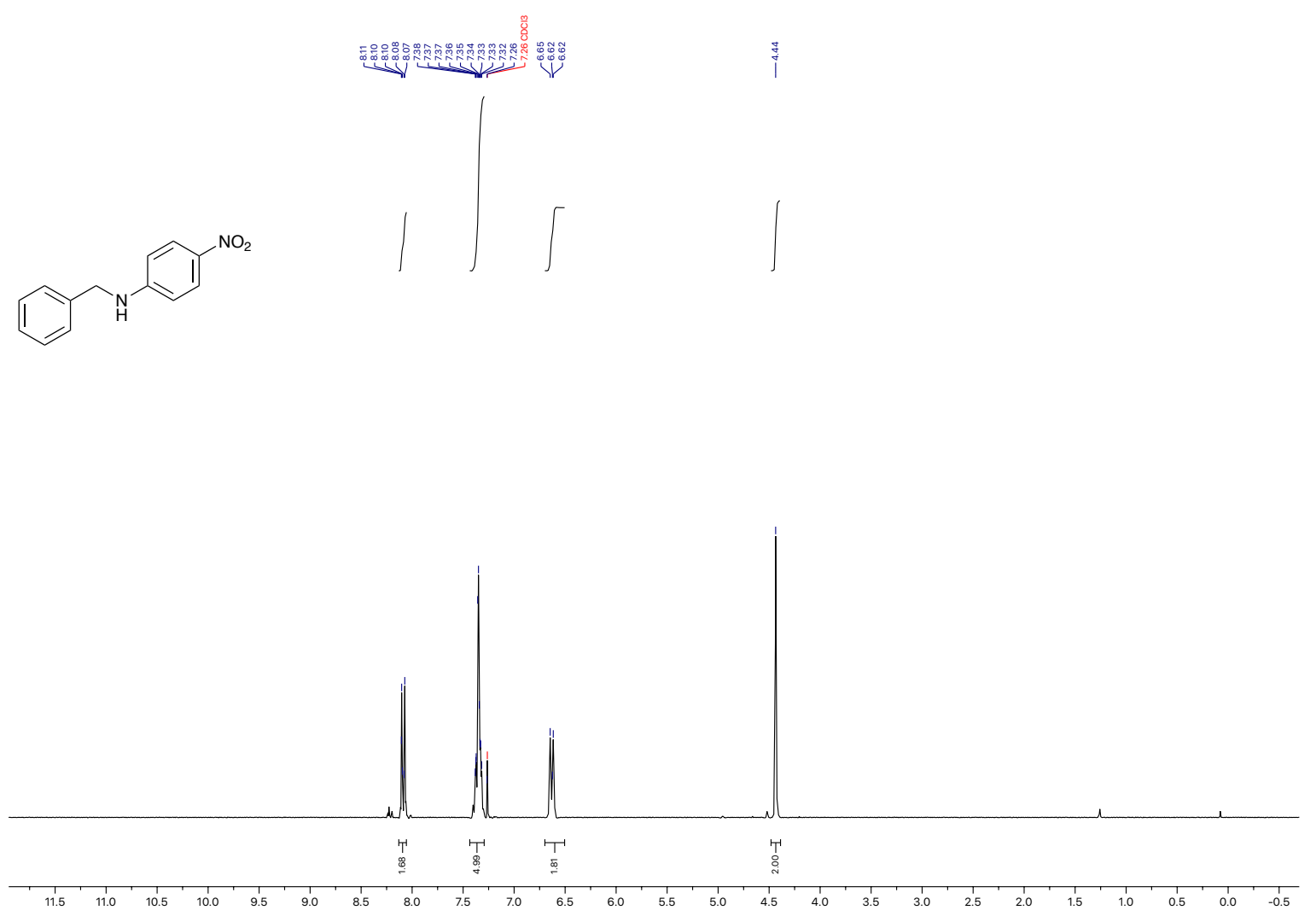

$\underline{300 \mathrm{MHz}}{ }^{1} \mathrm{H}$ NMR of $\mathrm{N}-b e n z y l-4-n i t r o a n i l i n e ~(4 a g) \underline{\text { in } \mathrm{CDCl}_{3}}$

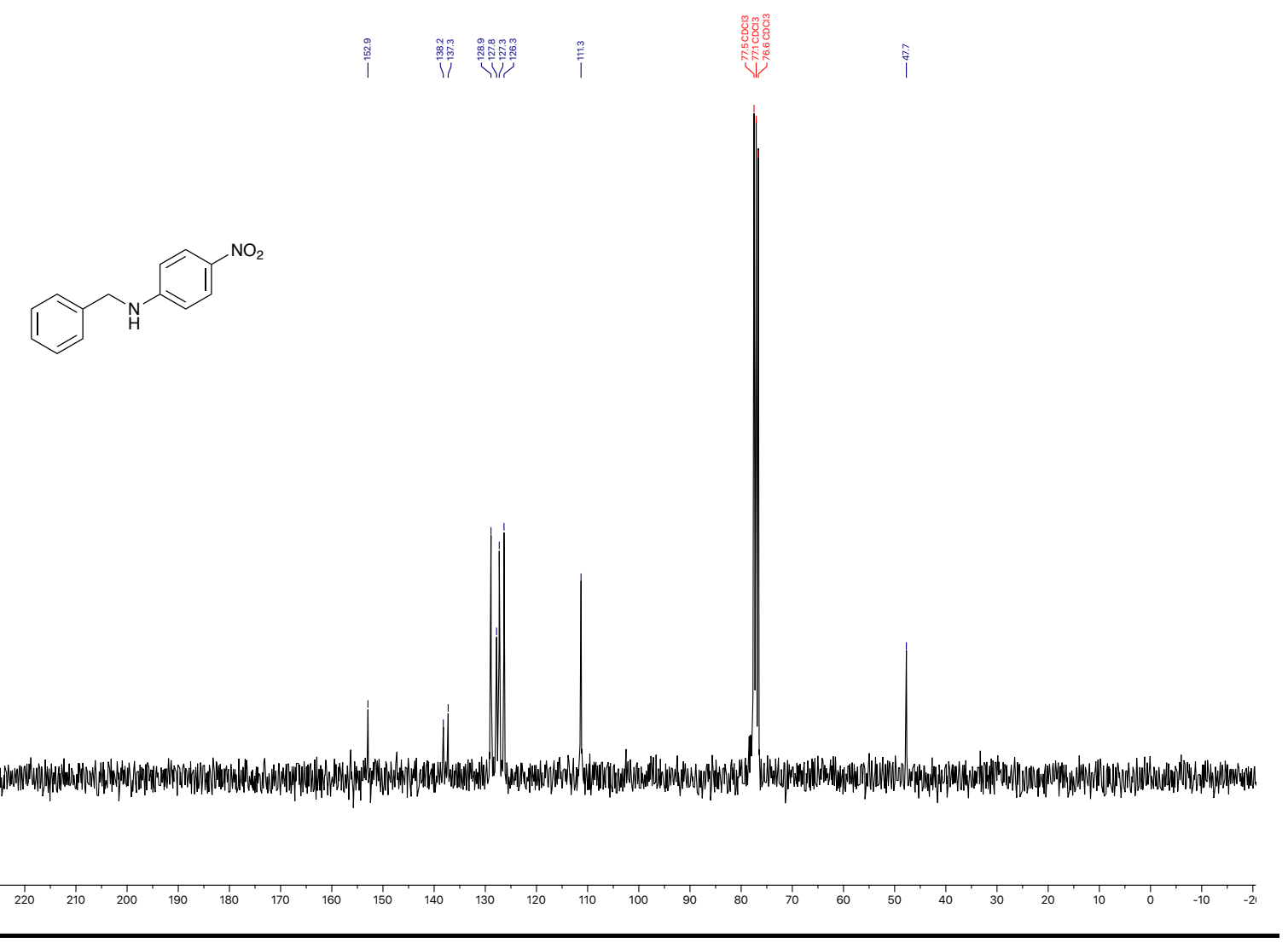

$\underline{75 \mathrm{MHz}}{ }^{13} \mathrm{C}\{\mathrm{H}\}$ NMR of $\mathrm{N}$-benzyl-4-nitroaniline (4ag) in $\mathrm{CDCl}_{3}$ 


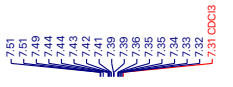

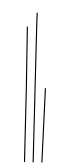

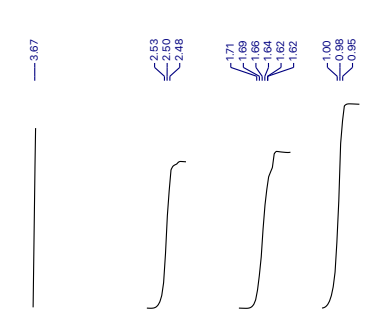
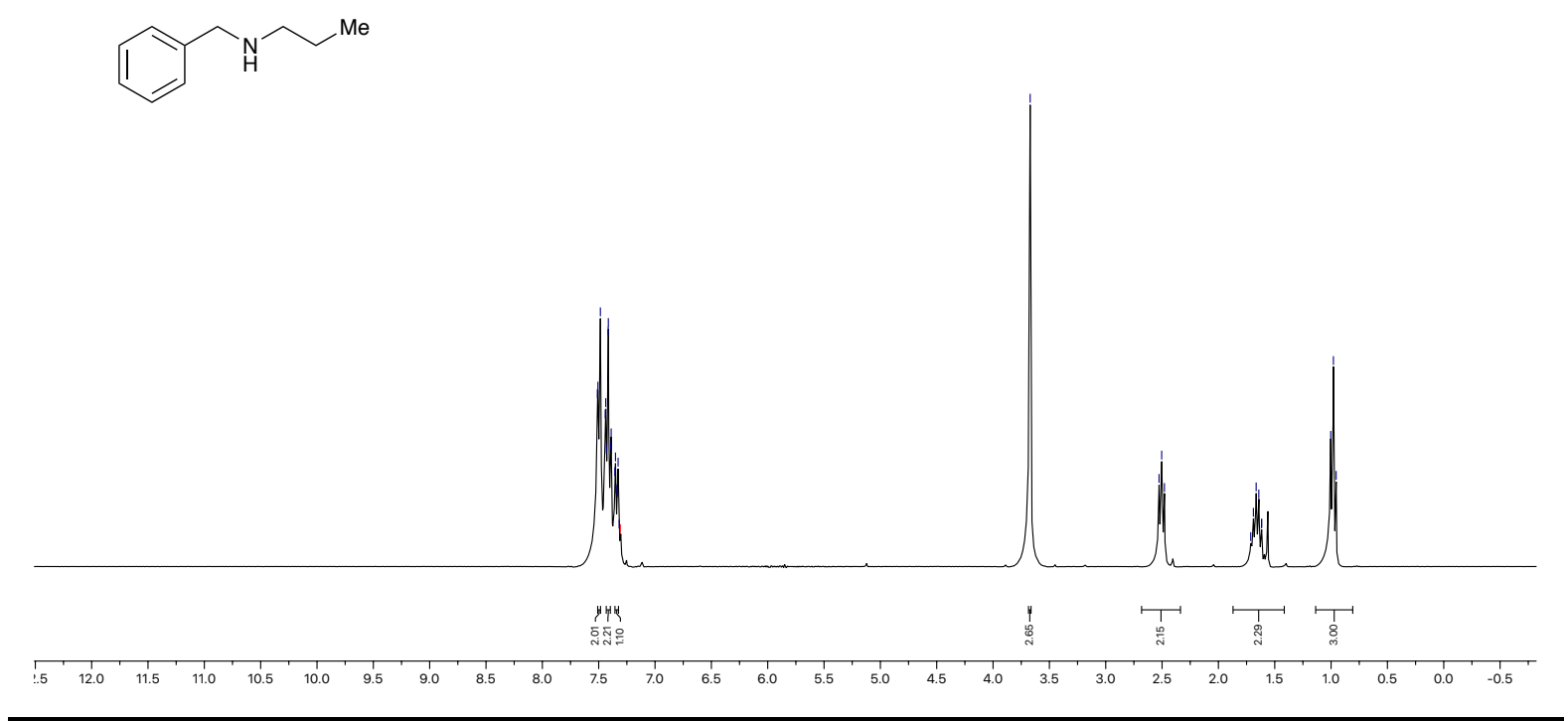

$\underline{300 \mathrm{MHz}}{ }^{1} \mathrm{H}$ NMR of $\mathrm{N}$-benzylpropan-1-amine (4ah) $\underline{\text { in } \mathrm{CDCl}_{3}}$

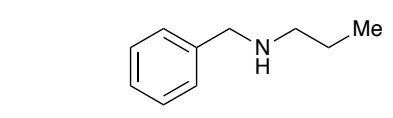

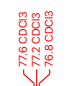

$\underset{1}{m}$

i̊n.
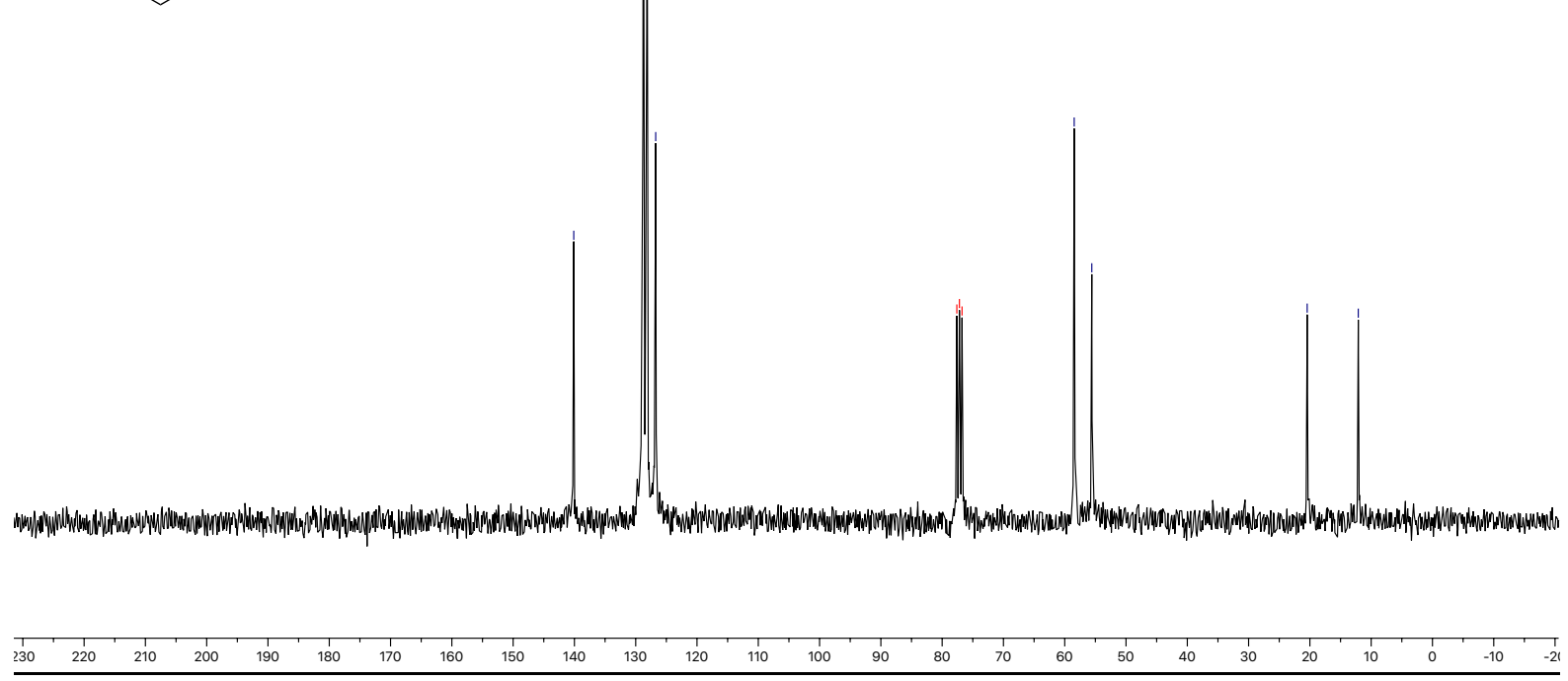

$\underline{75 \mathrm{MHz}^{13} \mathrm{C}\{\mathrm{H}\} \mathrm{NMR} \text { of }}$ N-benzylpropan-1-amine (4ah) in $\mathrm{CDCl}_{3}$

S18 


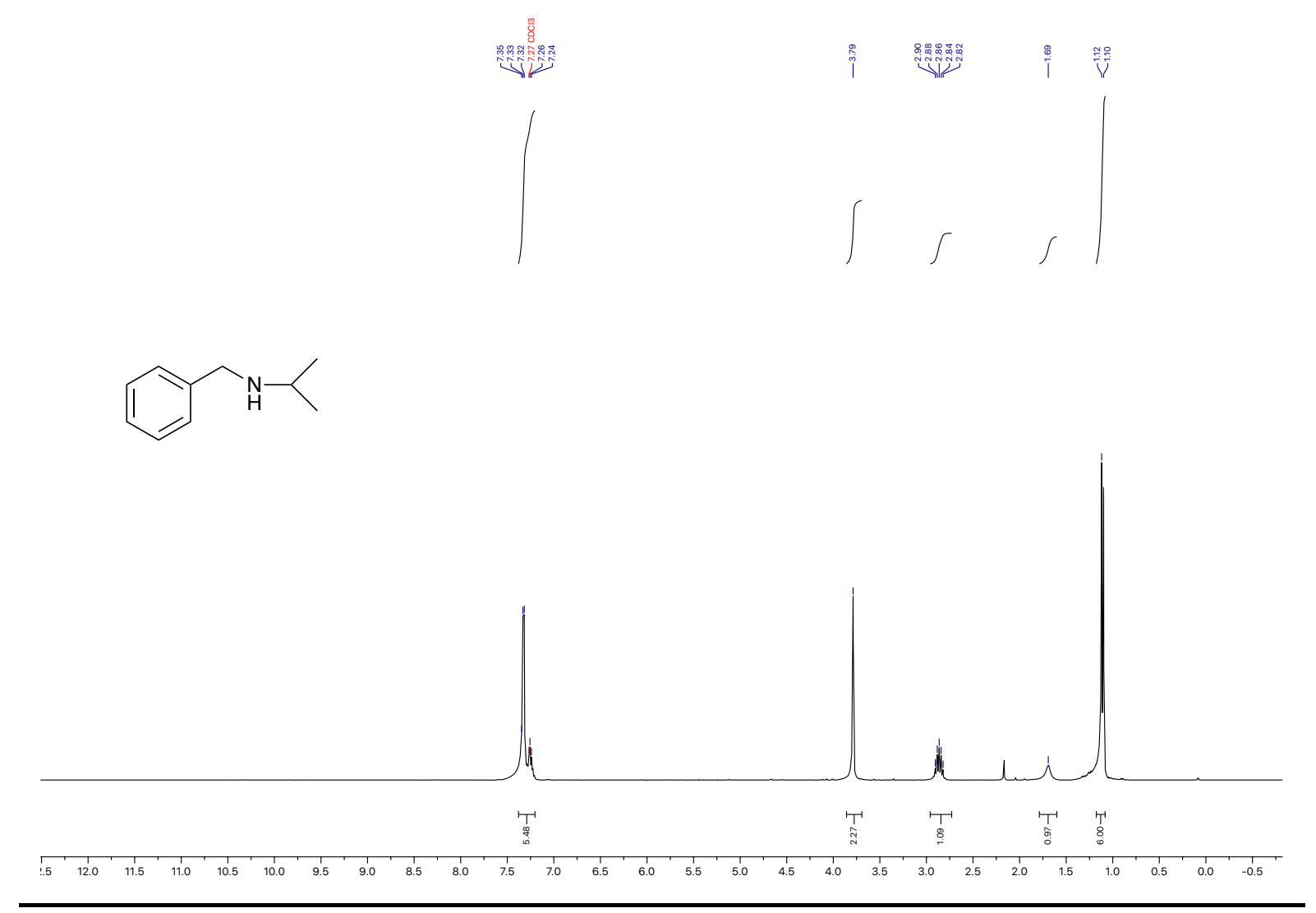

$\underline{300 \mathrm{MHz}^{1} \mathrm{H} \text { NMR of }}$ N-benzylpropan-2-amine (4ai) in $\underline{\mathrm{CDCl}_{3}}$

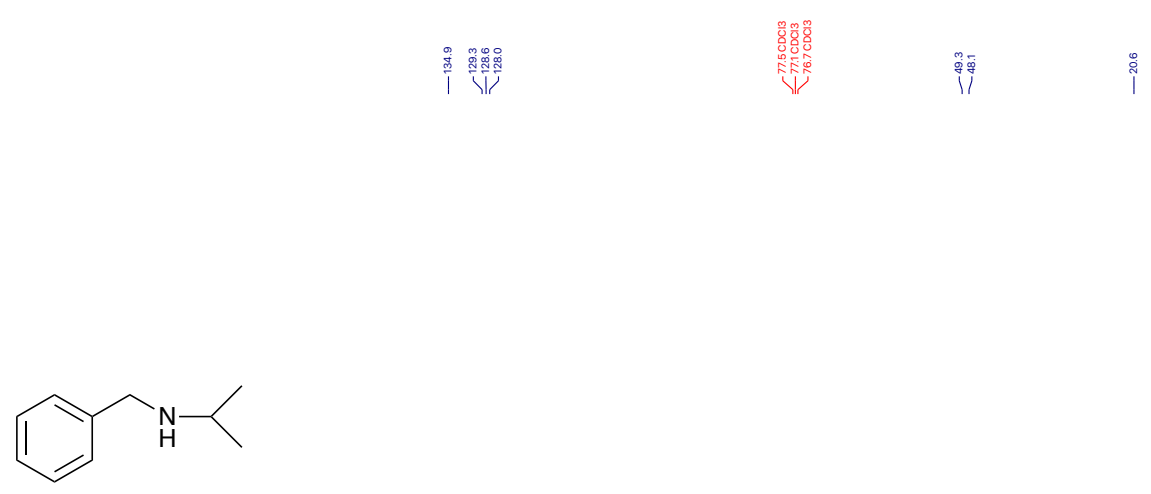

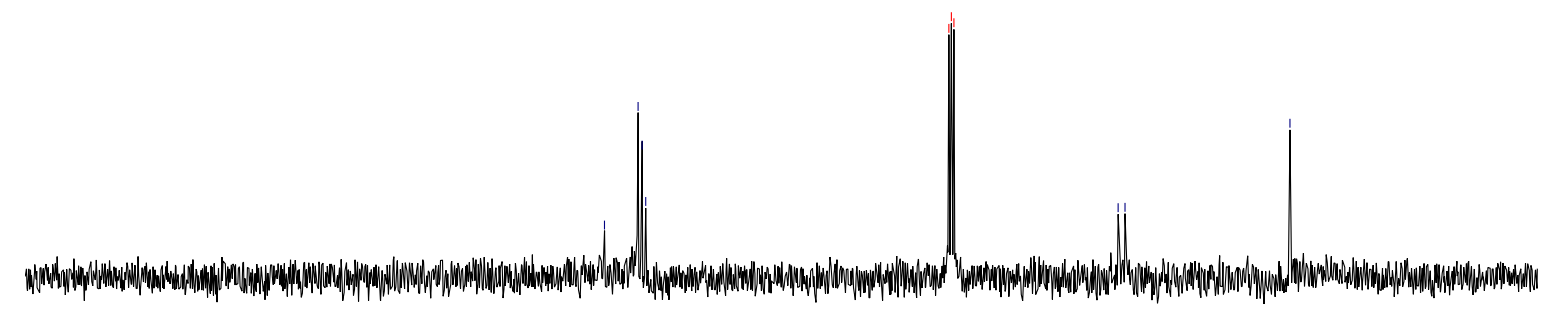

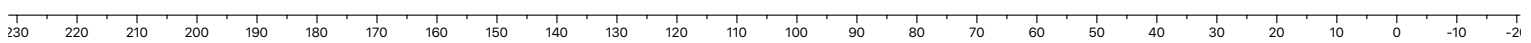

$\underline{75 \mathrm{MHz}}{ }^{13} \mathrm{C}\{\mathrm{H}\}$ NMR of $\mathrm{N}$-benzylpropan-2-amine (4ai) in $\underline{\mathrm{CDCl}_{3}}$ 


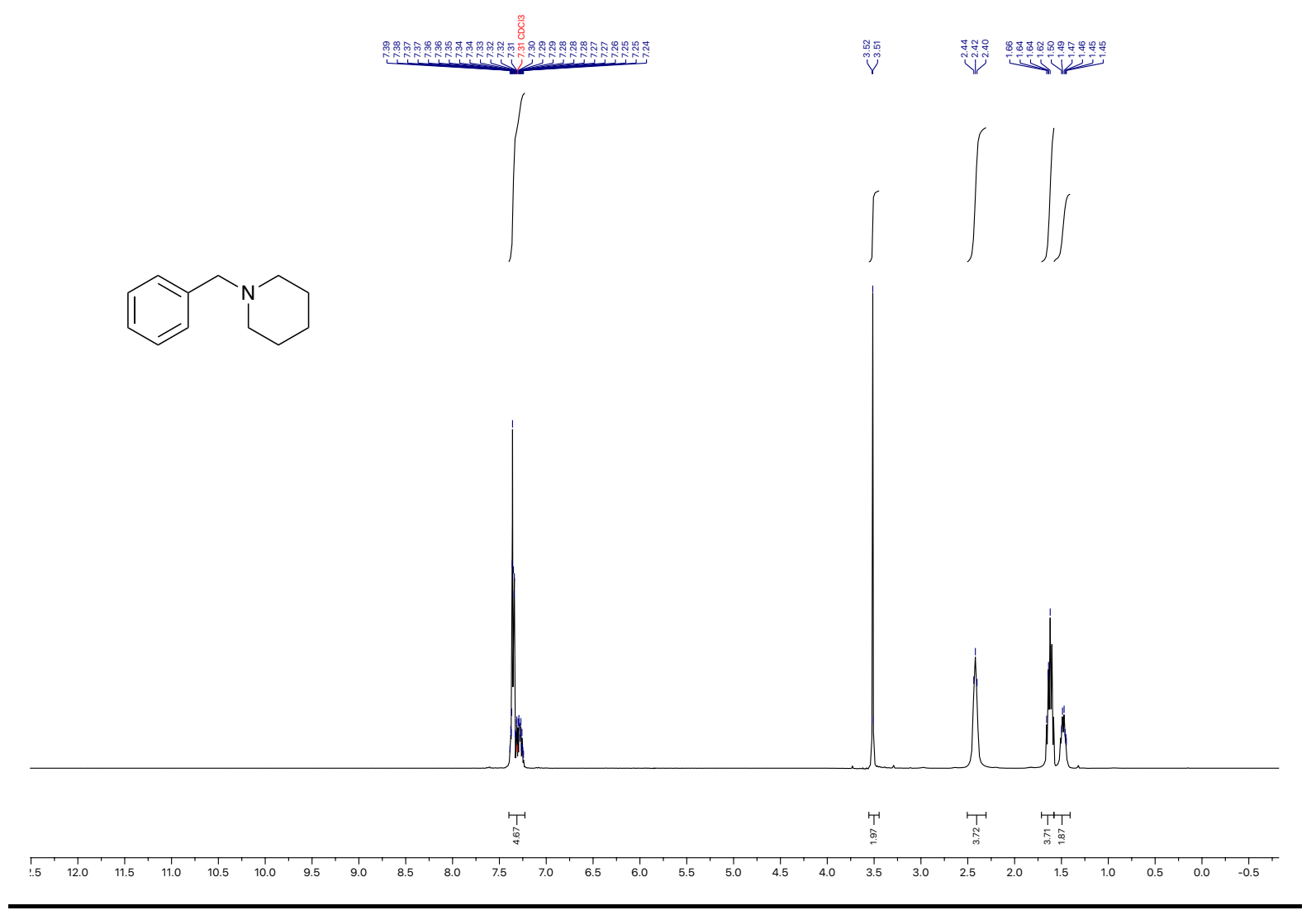

$\underline{300 \mathrm{MHz}}{ }^{1} \mathrm{H}$ NMR of 1 -benzylpiperidine in (4aj) $\underline{\mathrm{CDCl}_{3}}$
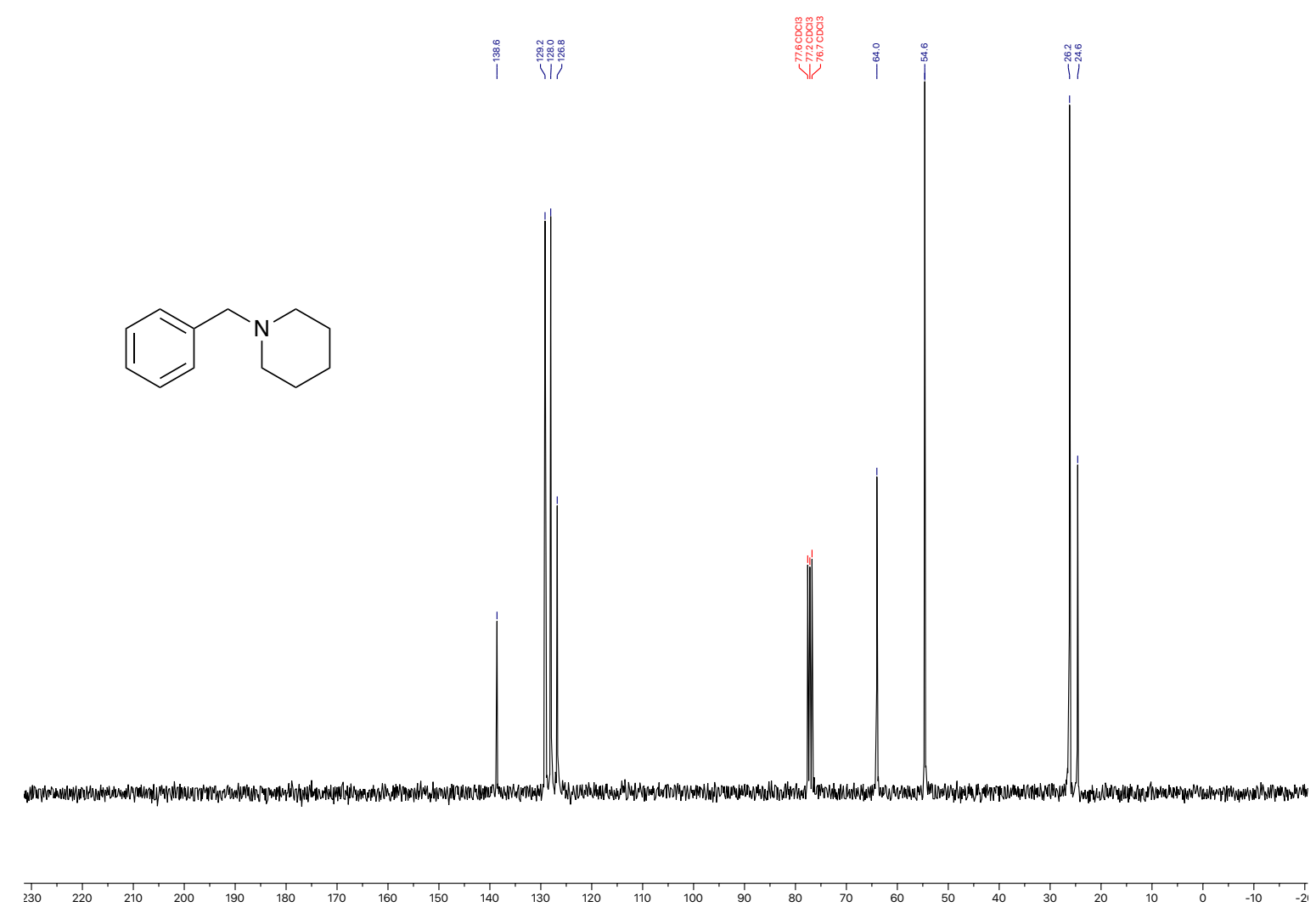

$\underline{75 \mathrm{MHz}}{ }^{13} \mathrm{C}\{\mathrm{H}\}$ NMR of $1-b e n z y l p i p e r i d i n e ~(4 a j) \underline{\text { in } \mathrm{CDCl}_{3}}$ 

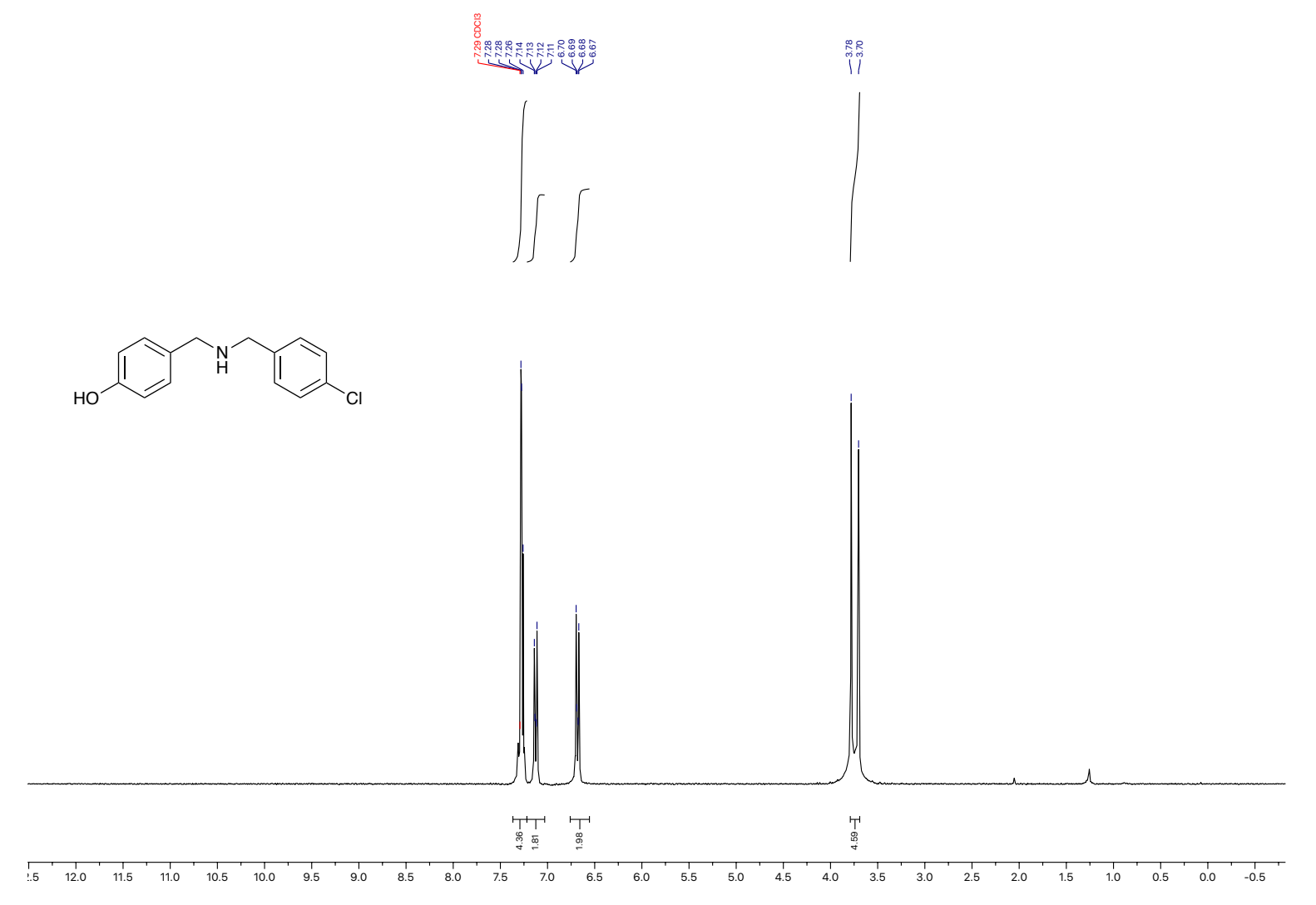

$\underline{300 \mathrm{MHz}}{ }^{1} \mathrm{H}$ NMR of $4-(((4-c h l o r o b e n z y l) a m i n o) m e t h y l) p h e n o l ~(4 b c) ~ \underline{\text { in } \mathrm{CDCl}_{3}}$
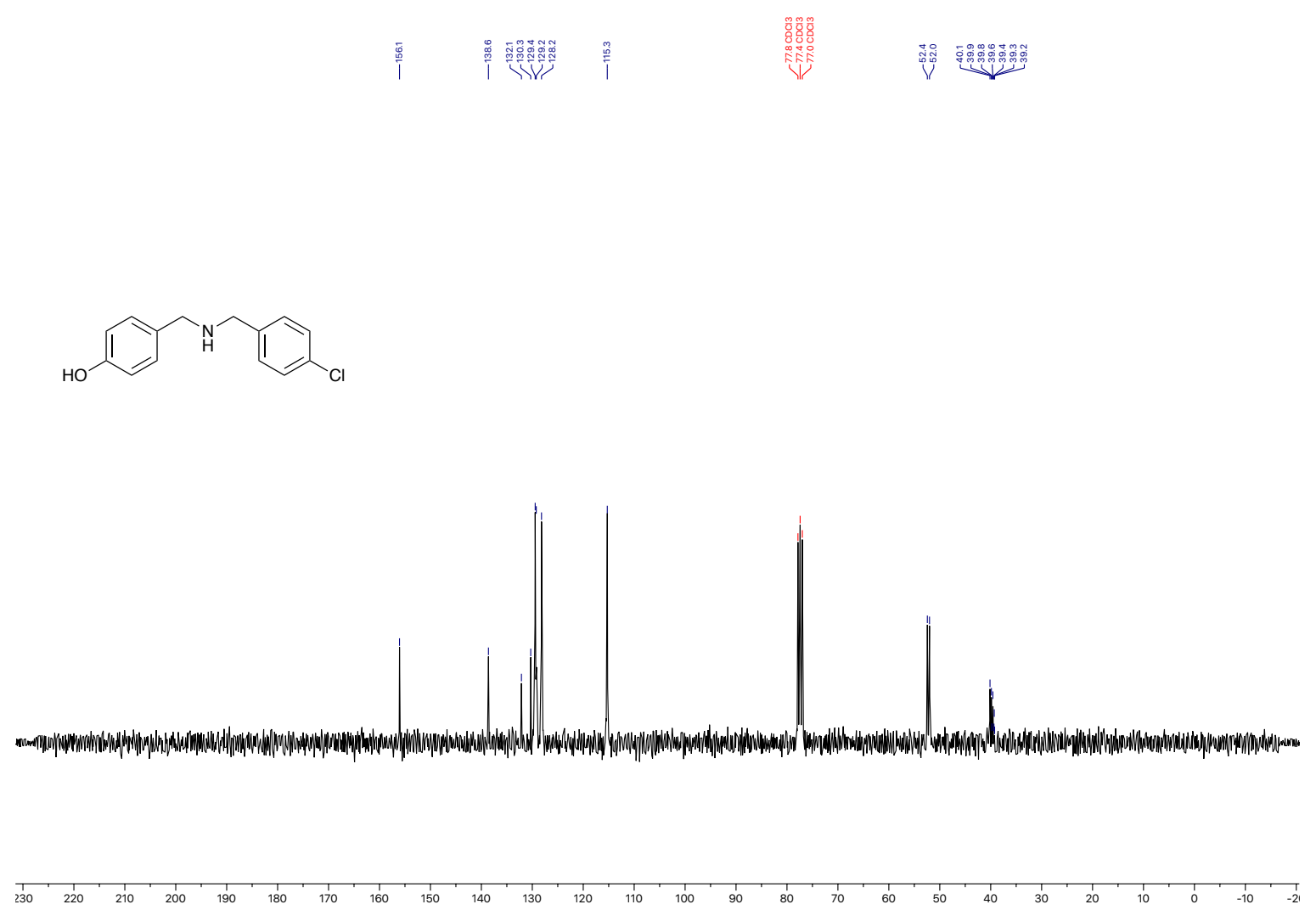

$\underline{75 \mathrm{MHz}}{ }^{13} \mathrm{C}\{\mathrm{H}\}$ NMR of 4-(((4-chlorobenzyl)amino)methyl)phenol (4bc) in $\mathrm{CDCl}_{3} / \mathrm{DMSO}-\mathrm{d} 6$ 


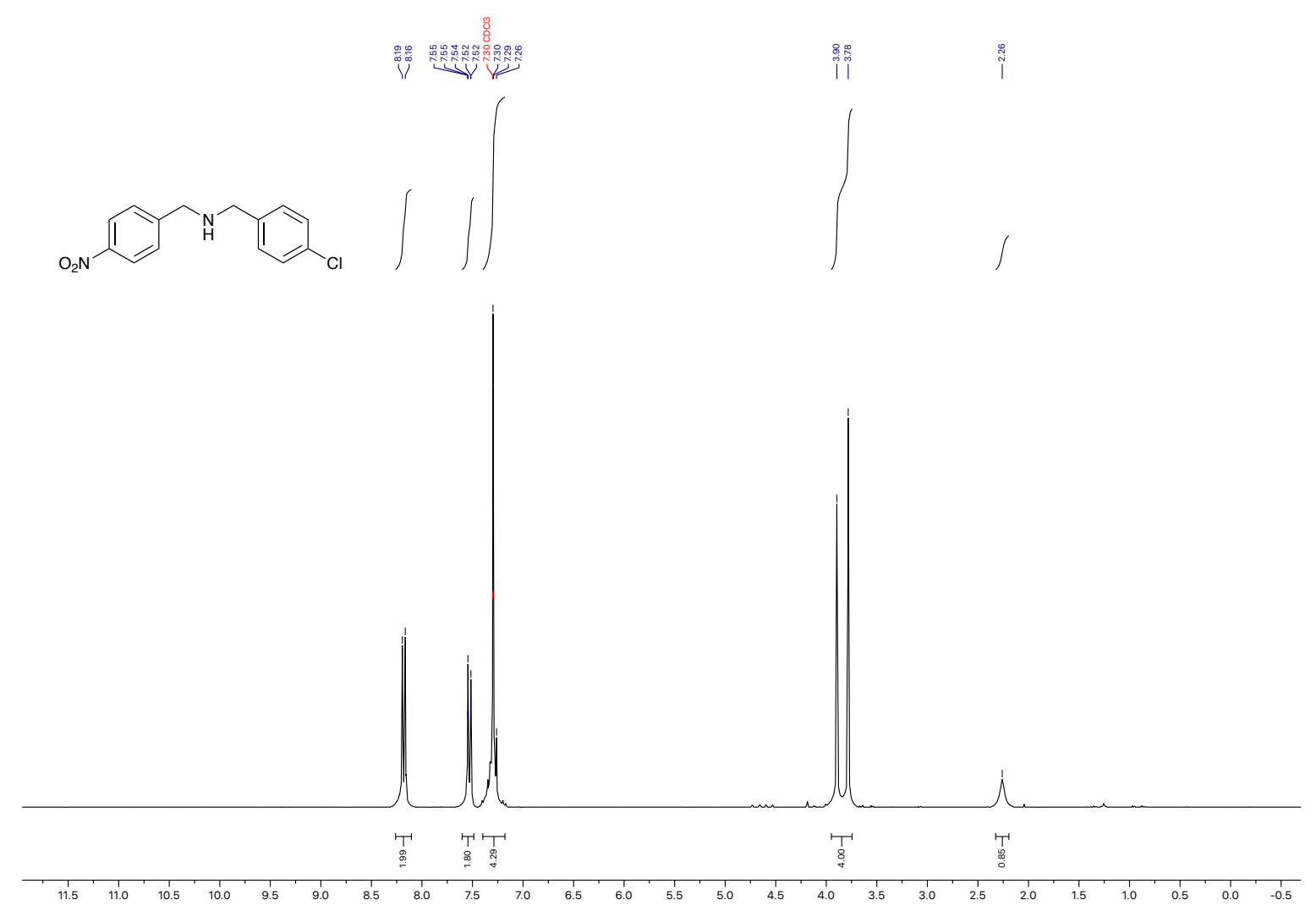

$\underline{300 \mathrm{MHz}}{ }^{1} \mathrm{H}$ NMR of $\mathrm{N}-(4-c h l o r o b e n z y l)-1-(4-n i t r o p h e n y l) m e t h a n a m i n e ~(4 \mathrm{cc}) \underline{\text { in } \mathrm{CDCl}_{3}}$

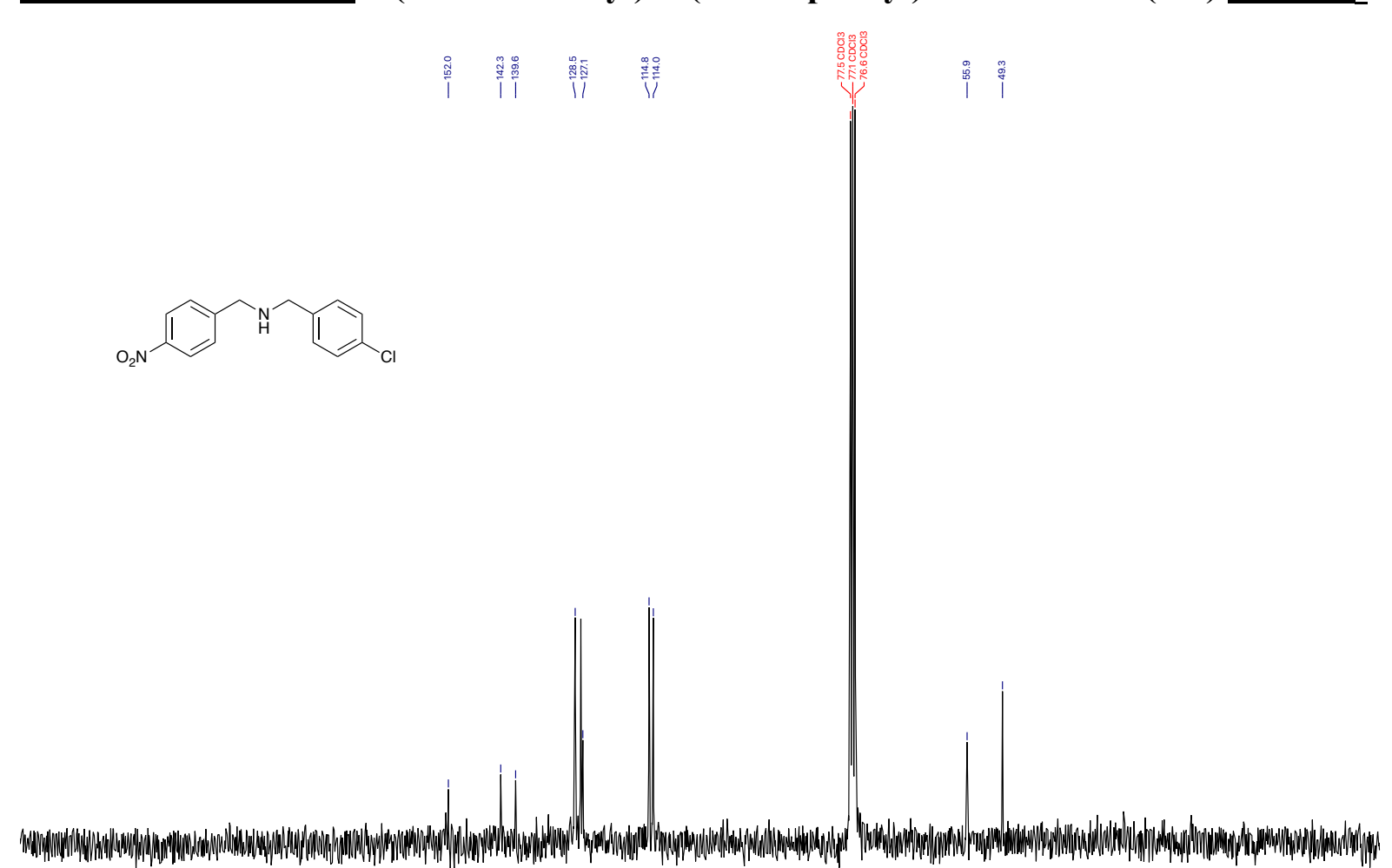

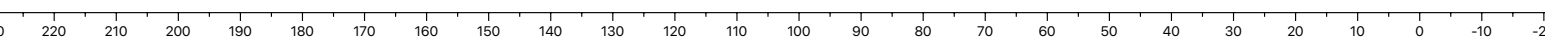

$\underline{75 \mathrm{MHz}}{ }^{13} \mathrm{C}\{\mathrm{H}\}$ NMR of $\mathrm{N}$-(4-chlorobenzyl)-1-(4-nitrophenyl)methanamine (4cc) in $\underline{\mathrm{CDCl}_{3}}$ 


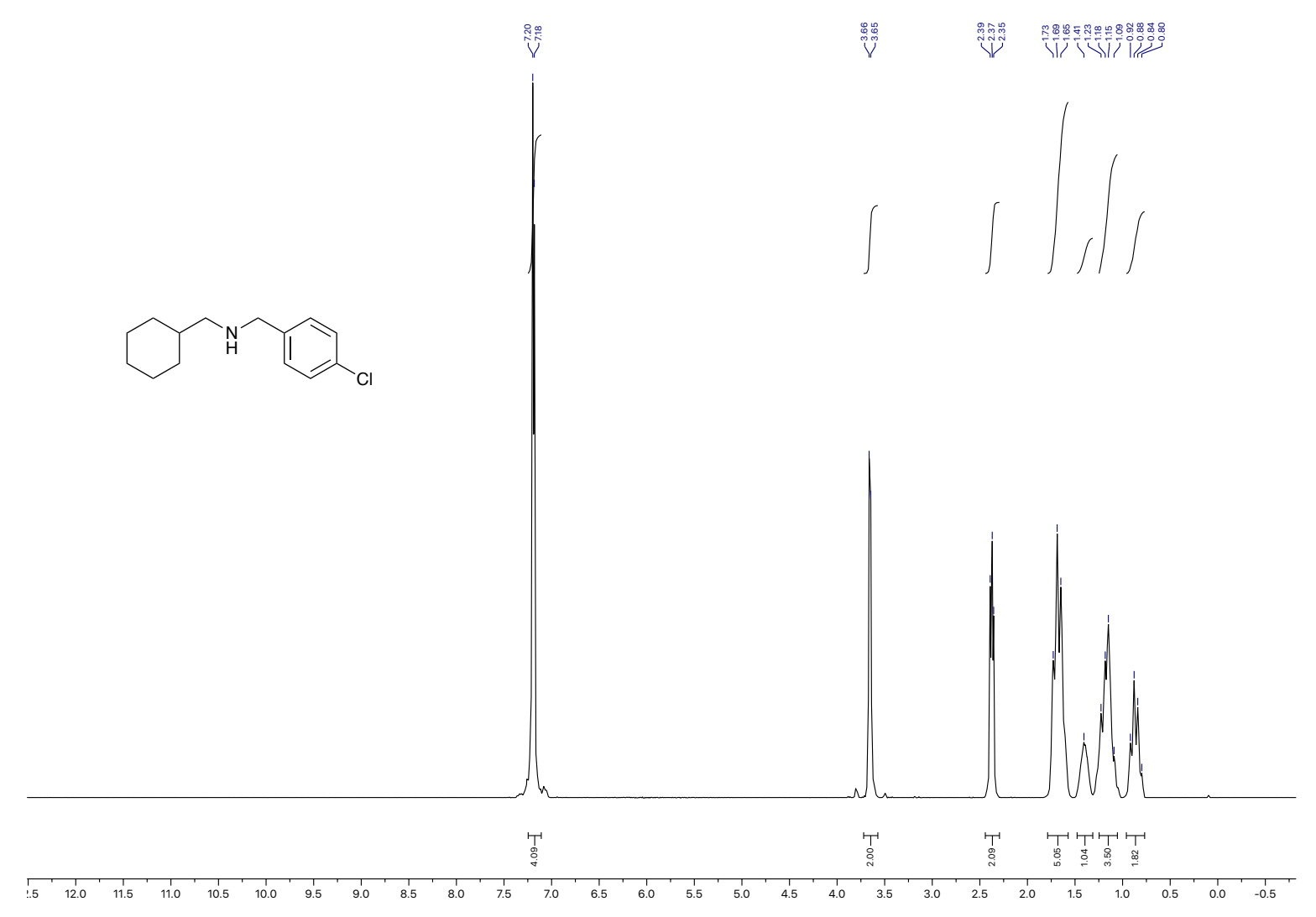

$\underline{300 ~ M H z}{ }^{1} \mathrm{H}$ NMR of $\mathrm{N}-(4-c h l o r o b e n z y l)-1-c y c l o h e x y l m e t h a n a m i n e ~(4 d c) ~ \underline{\text { in } \mathrm{CDCl}_{3}}$ 1
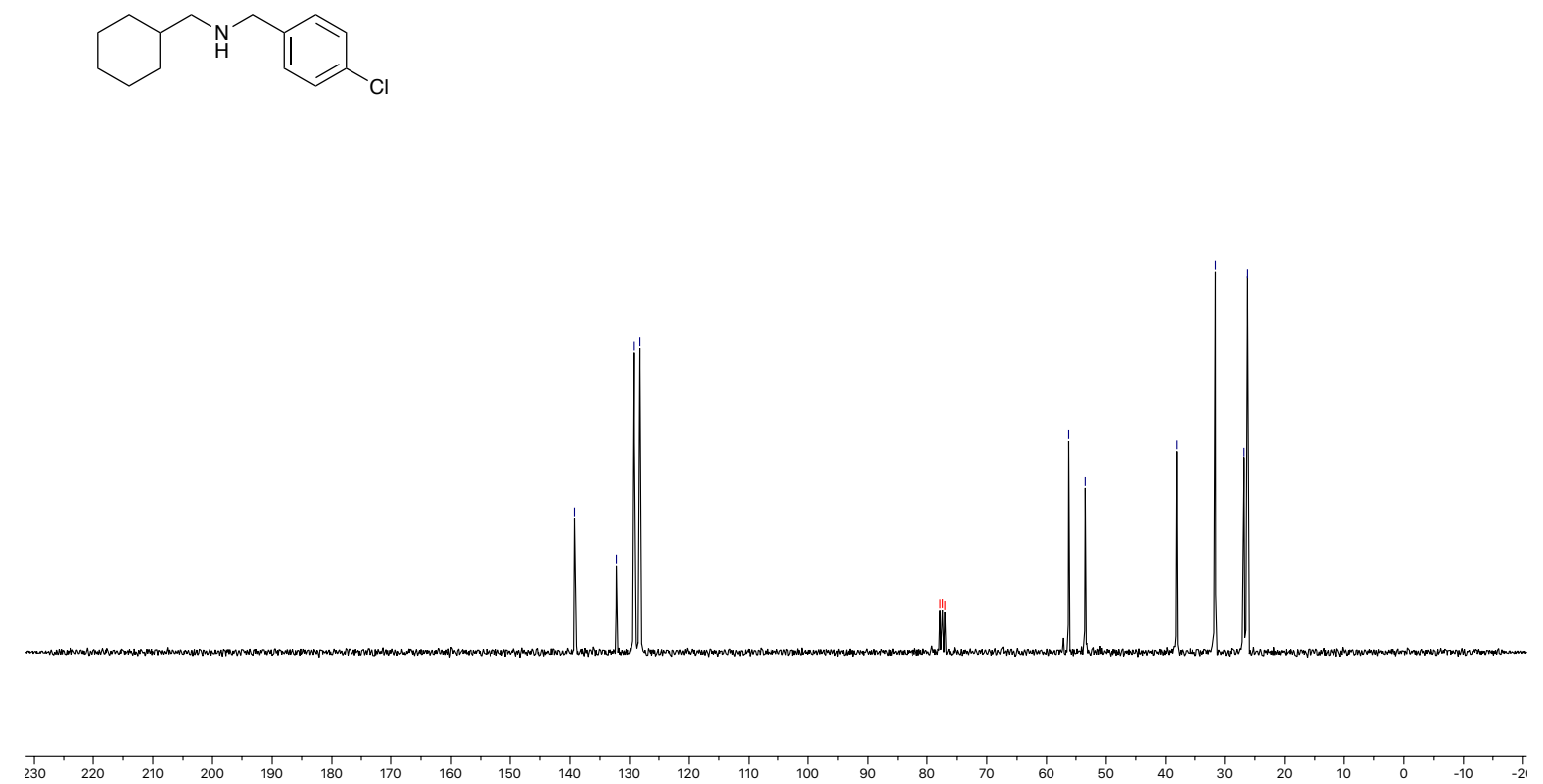

$\underline{75 \mathrm{MHz}}{ }^{13} \mathrm{C}\{\mathrm{H}\}$ NMR of $\mathrm{N}-(4-c h l o r o b e n z y l)-1$-cyclohexylmethanamine (4dc) $\underline{\text { in } \mathrm{CDCl}_{3}}$ 


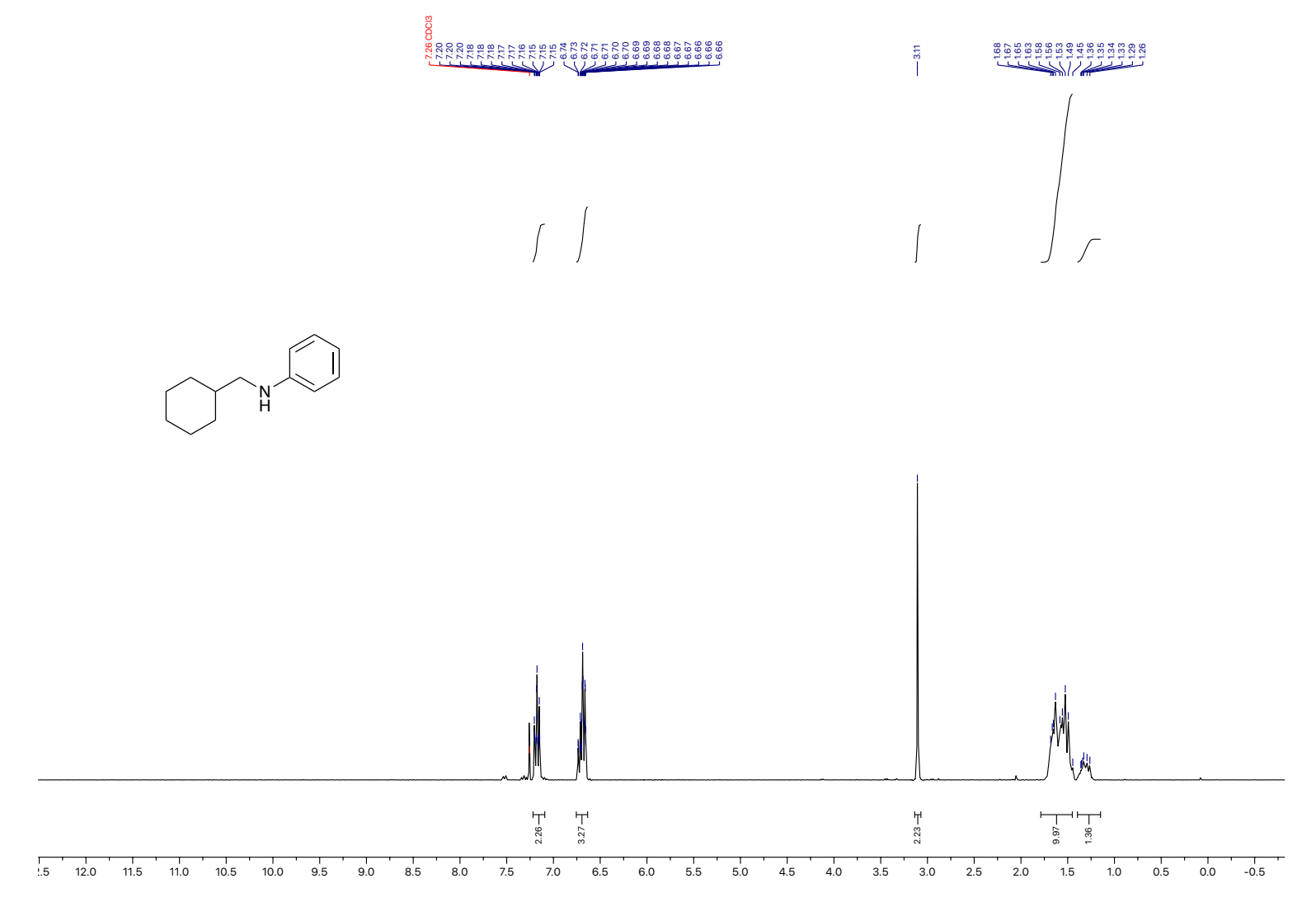

$\underline{300 \mathrm{MHz}^{1} \mathrm{H} \text { NMR of }} \mathrm{N}$-(cyclohexylmethyl)aniline (4dd) in $\underline{\mathrm{CDCl}_{3}}$
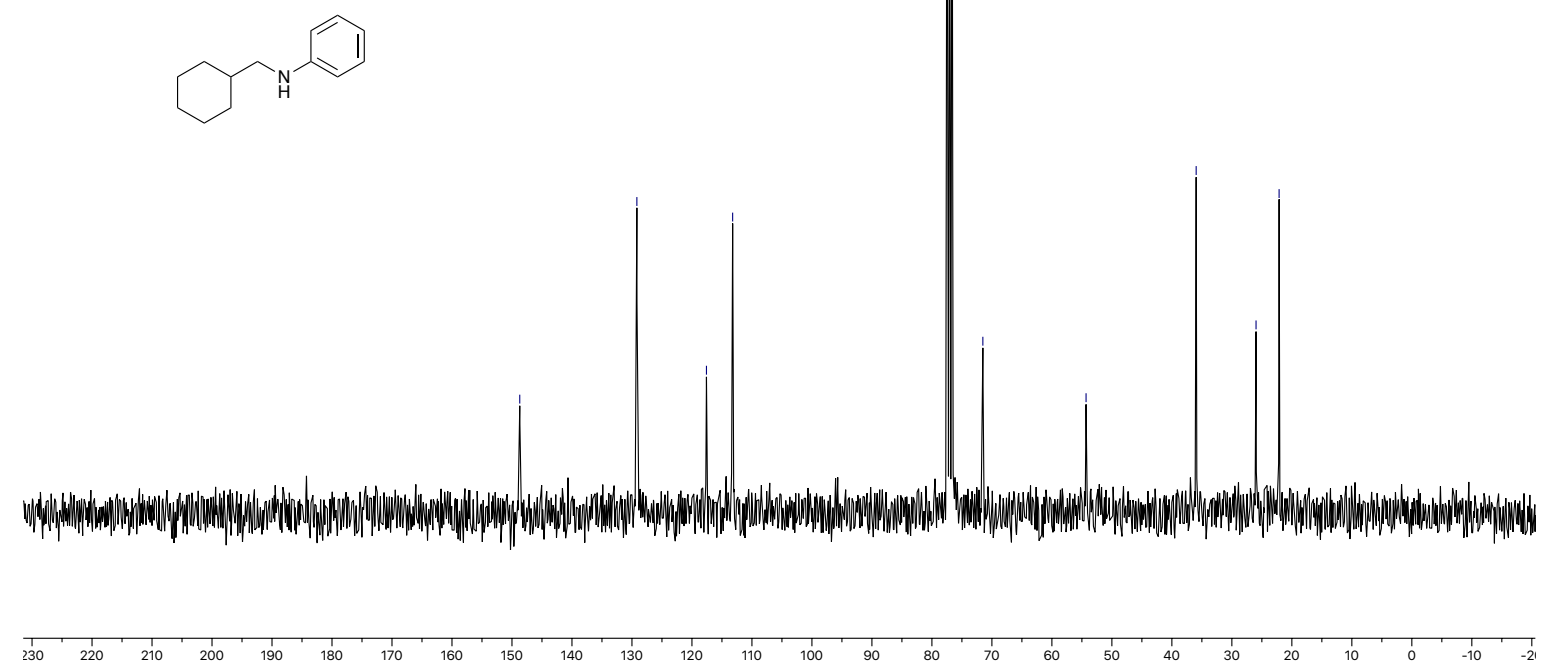

$\underline{75 \mathrm{MHz}}{ }^{13} \mathrm{C}\{\mathrm{H}\}$ NMR of $\mathrm{N}$-(cyclohexylmethyl)aniline (4dd) in $\mathrm{CDCl}_{3}$ 


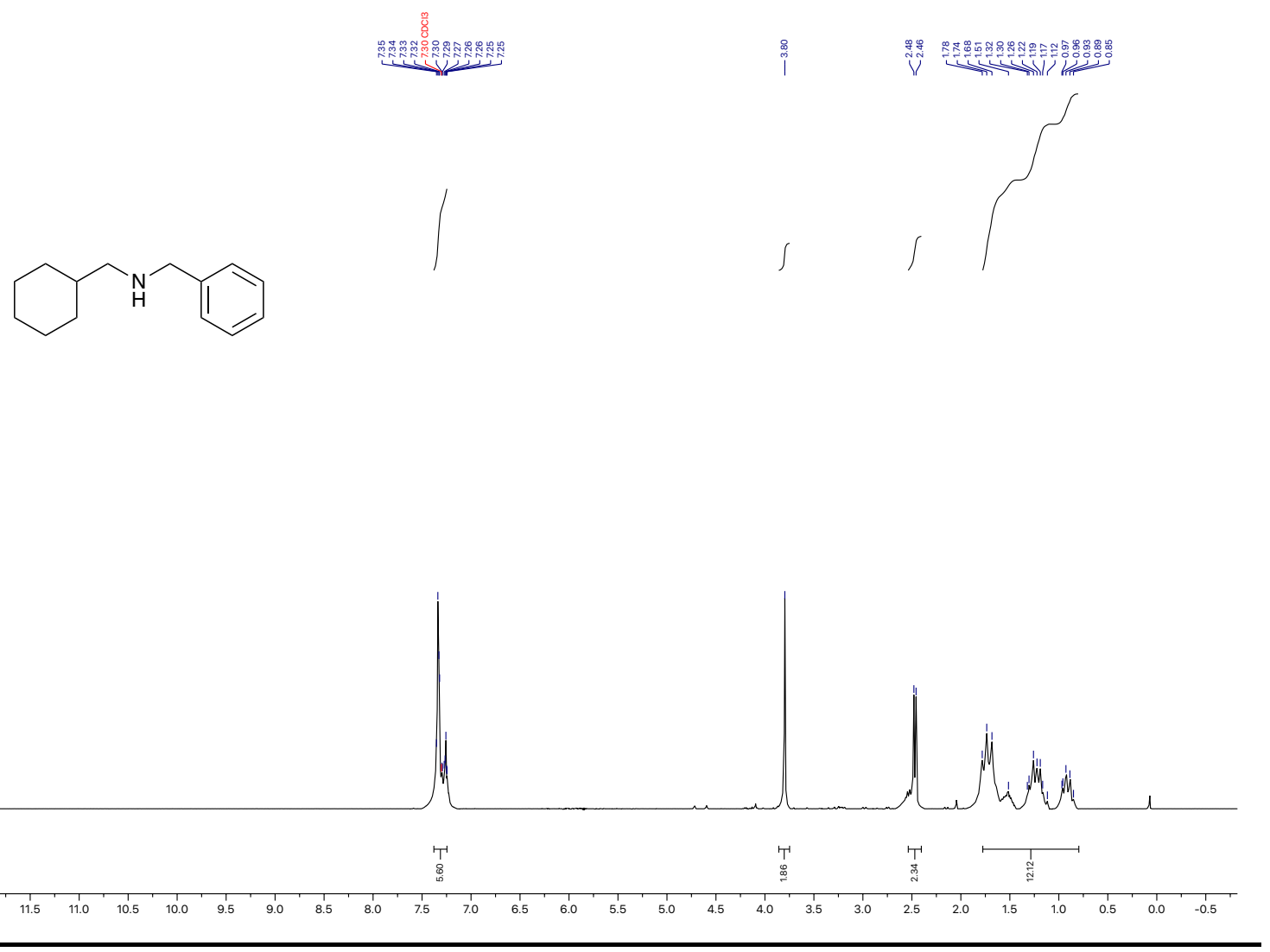

$\underline{300} \mathrm{MHz}^{1} \mathrm{H}$ NMR of $\mathrm{N}-$ benzyl-1-cyclohexylmethanamine (4da) in $\underline{\mathrm{CDCl}_{3}}$

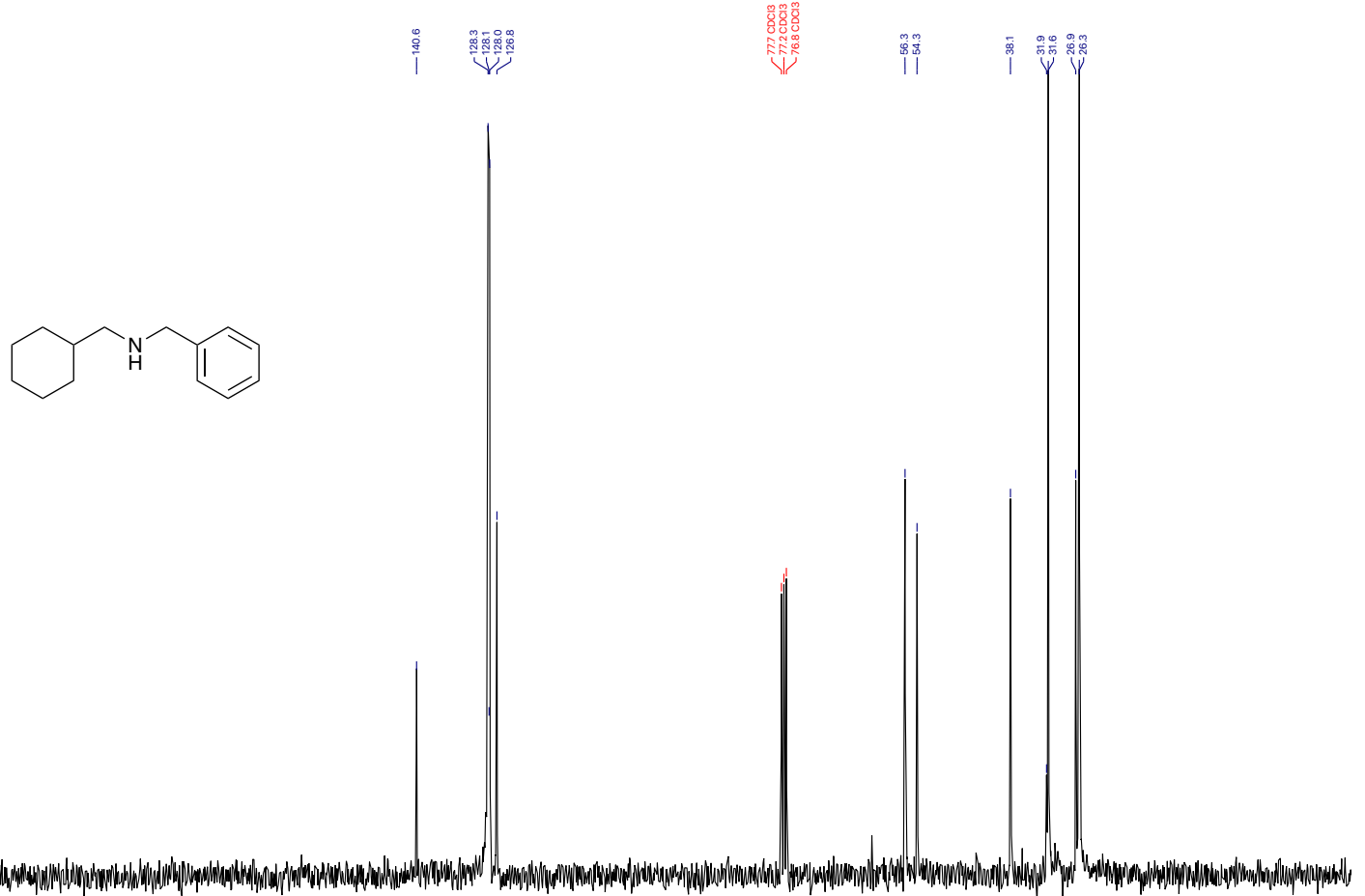

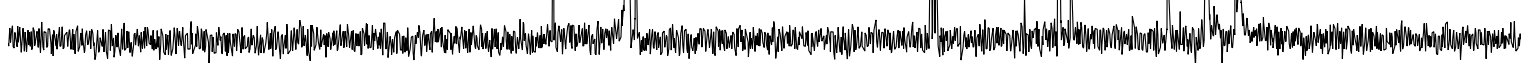

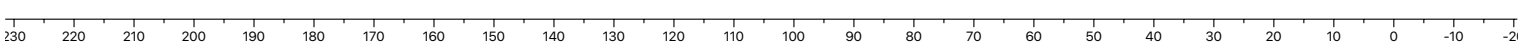

$\underline{75 \mathrm{MHz}}{ }^{13} \mathrm{C}\{\mathrm{H}\}$ NMR of $\mathrm{N}$-benzyl-1-cyclohexylmethanamine (4da) in $\mathrm{CDCl}_{3}$ 


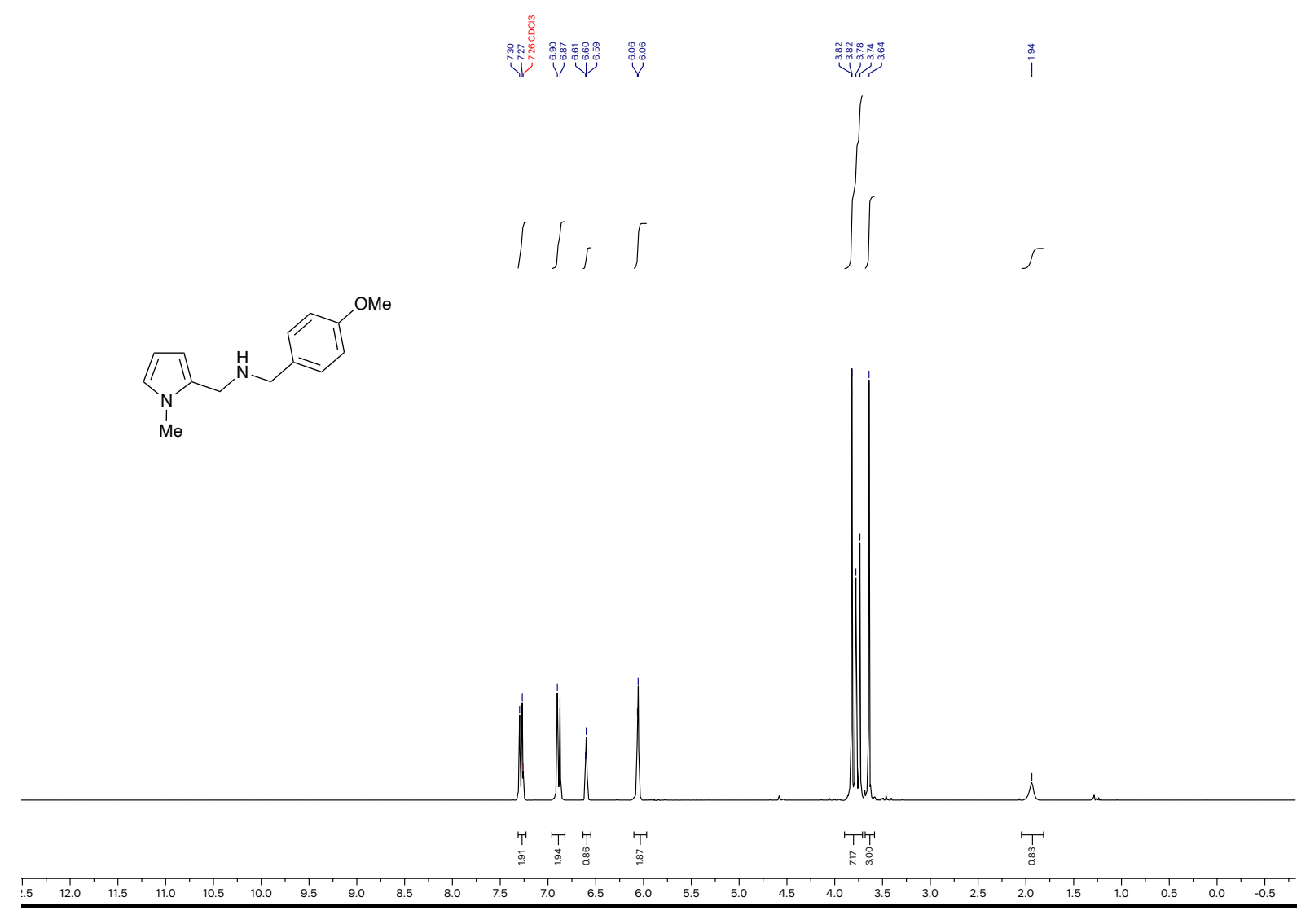

$\underline{300 ~ M H z}{ }^{1} \mathrm{H}$ NMR of $\mathrm{N}$-(4-methoxybenzyl)-1-(1-methyl-1H-pyrrol-2-yl)methanamine (4eb) in $\underline{\mathrm{CDCl}_{3}}$
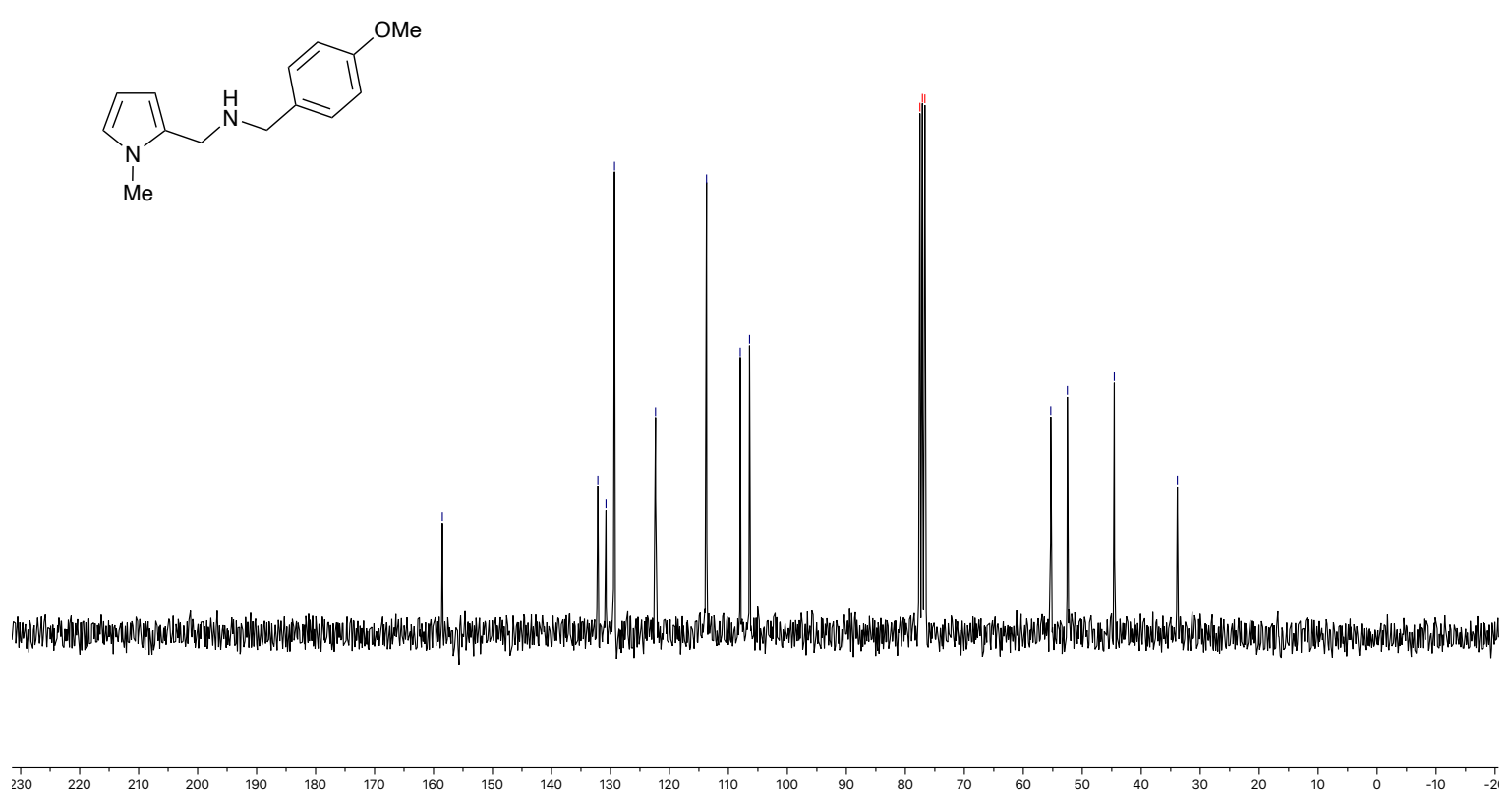

$75 \mathrm{MHz}{ }^{13} \mathrm{C}\{\mathrm{H}\}$ NMR of N-(4-methoxybenzyl)-1-(1-methyl-1H-pyrrol-2-

yl)methanamine (4eb) in $\underline{\mathrm{CDCl}_{3}}$ 


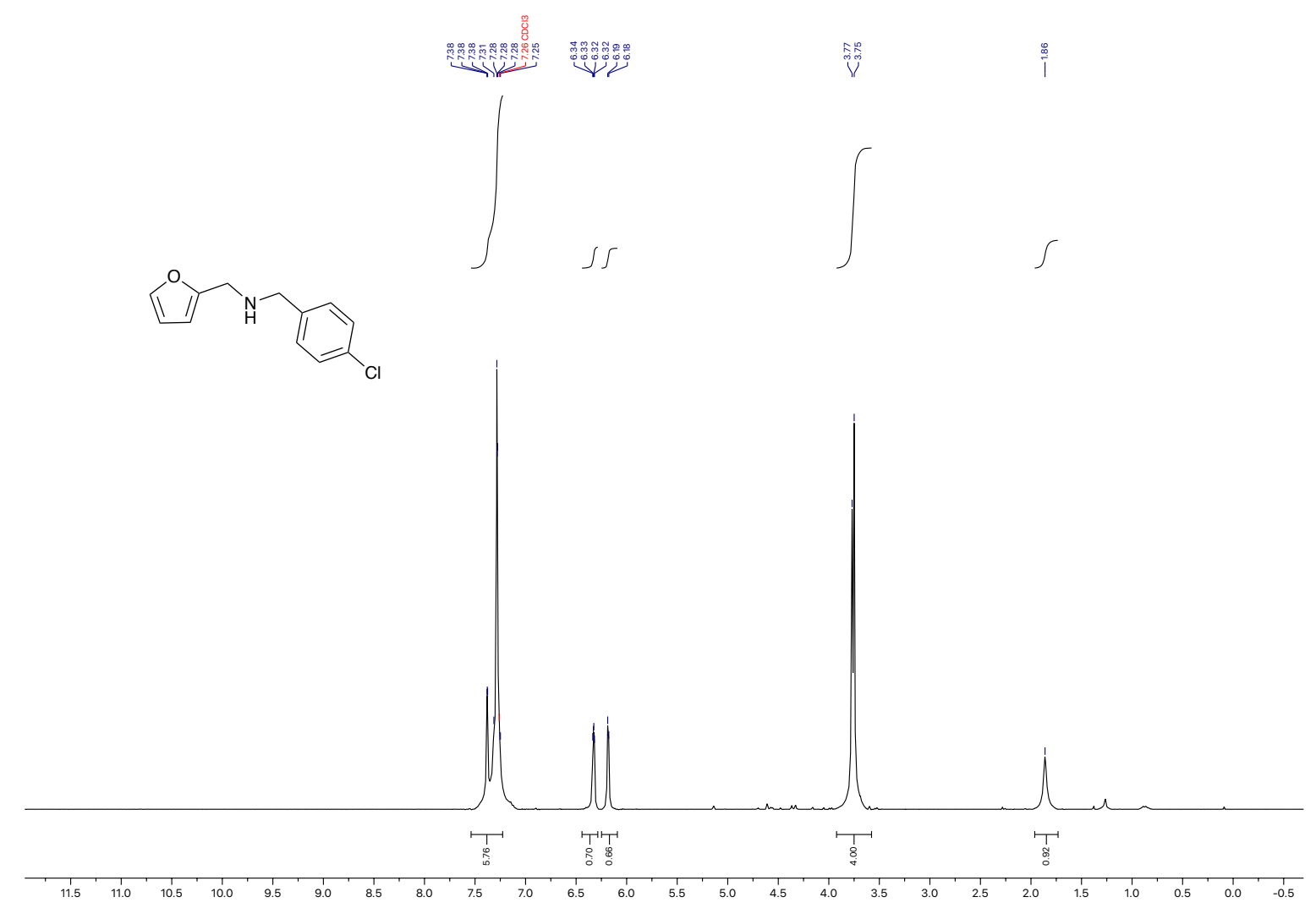

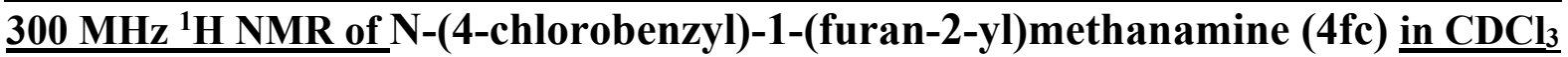
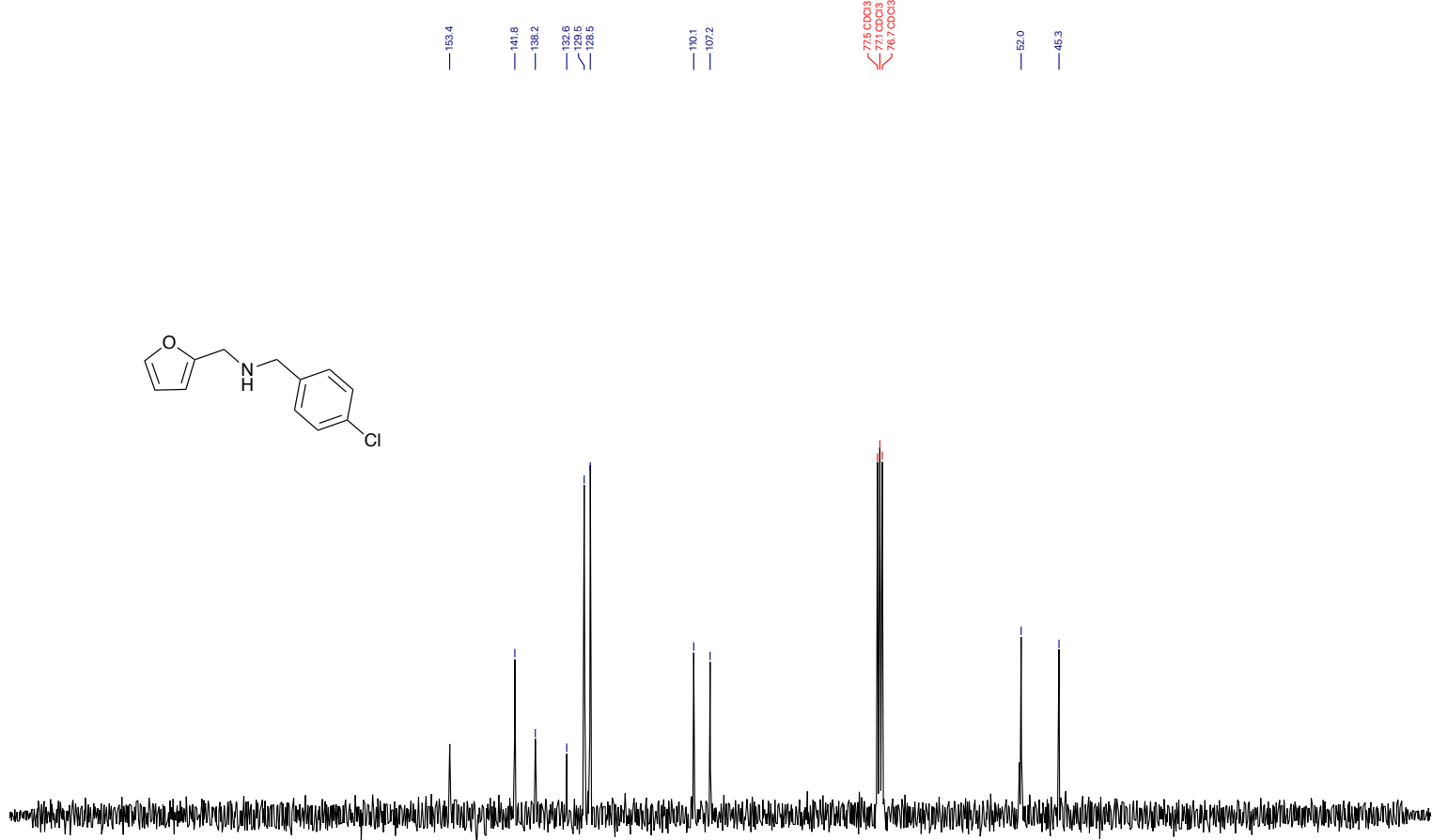

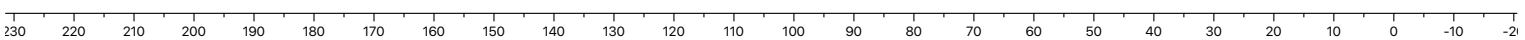

$\underline{75 \mathrm{MHz}}{ }^{13} \mathrm{C}\{\mathrm{H}\}$ NMR of $\mathrm{N}$-(4-chlorobenzyl)-1-(furan-2-yl)methanamine (4fc) in $\underline{\mathrm{CDCl}_{3}}$ 


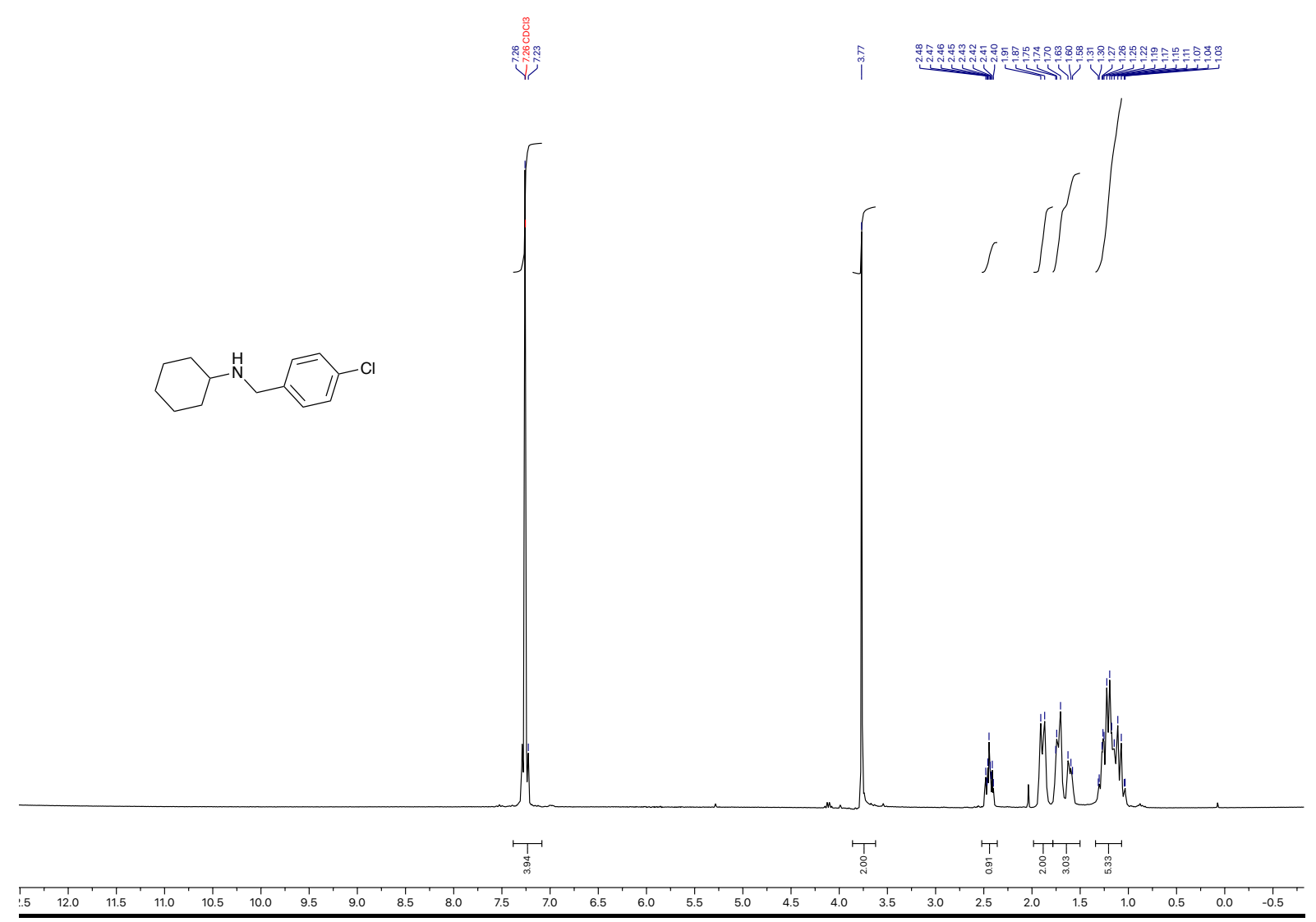

$\underline{300 \mathrm{MHz}}{ }^{1} \mathrm{H}$ NMR of $\mathrm{N}-(4-c h l o r o b e n z y l) c y c l o h e x a n a m i n e ~(4 \mathrm{gc}) \underline{\text { in } \mathrm{CDCl}_{3}}$

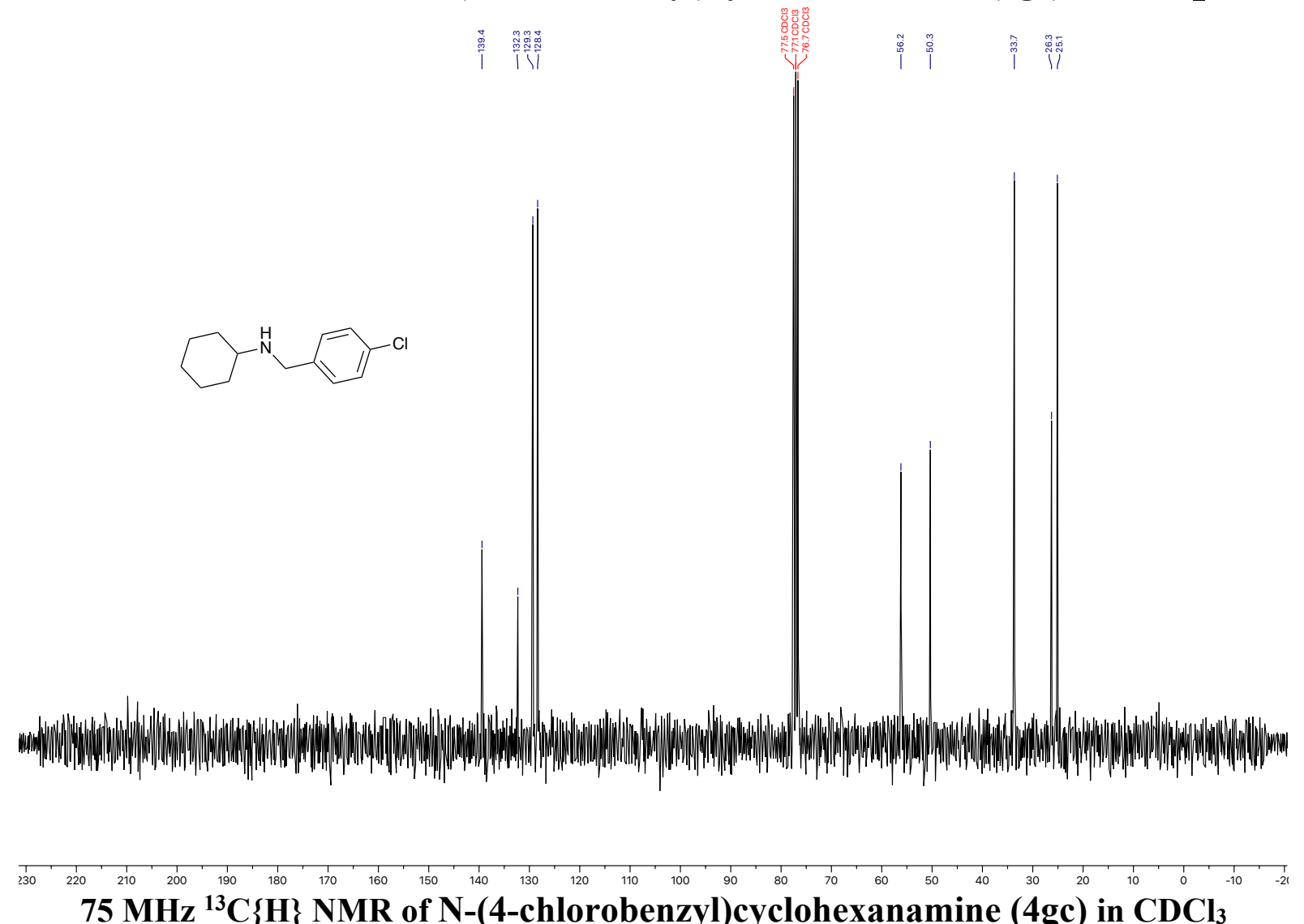

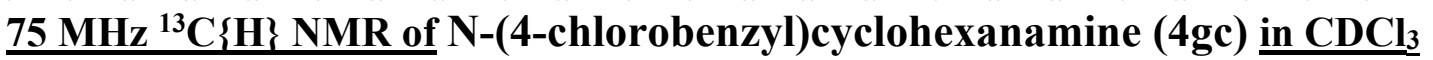




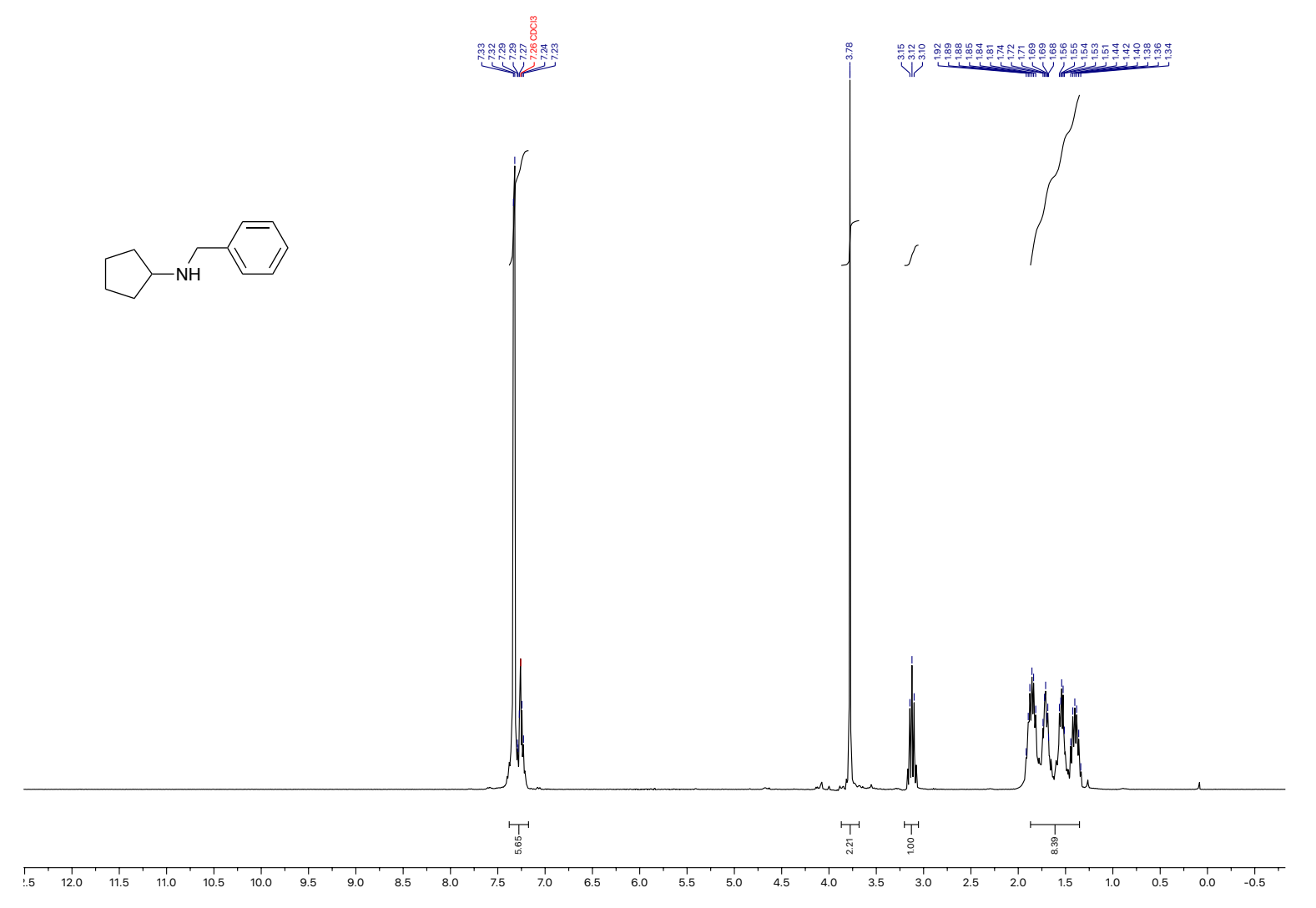

$\underline{300} \mathrm{MHz}^{1} \mathrm{H}$ NMR of $\mathrm{N}-$ benzylcyclopentanamine (4ha) in $\mathrm{CDCl}_{3}$

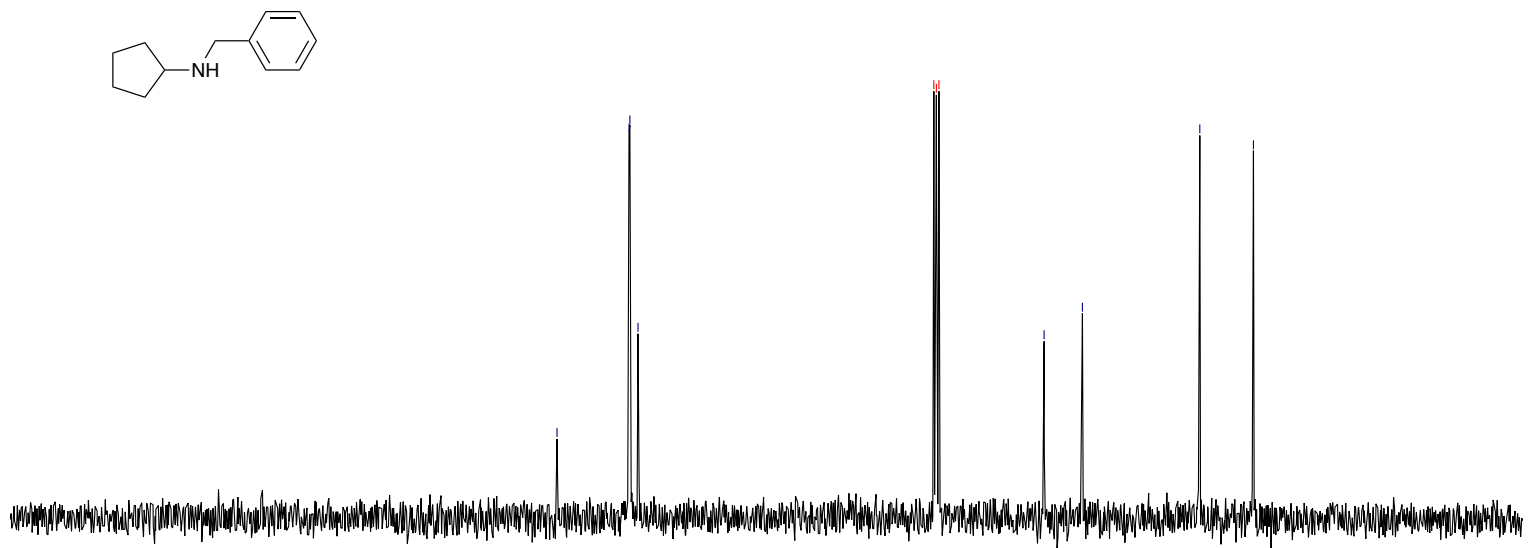

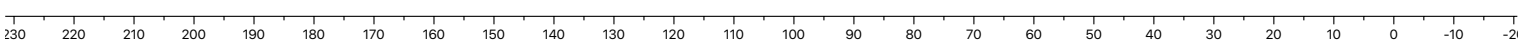

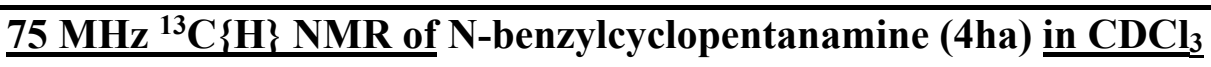




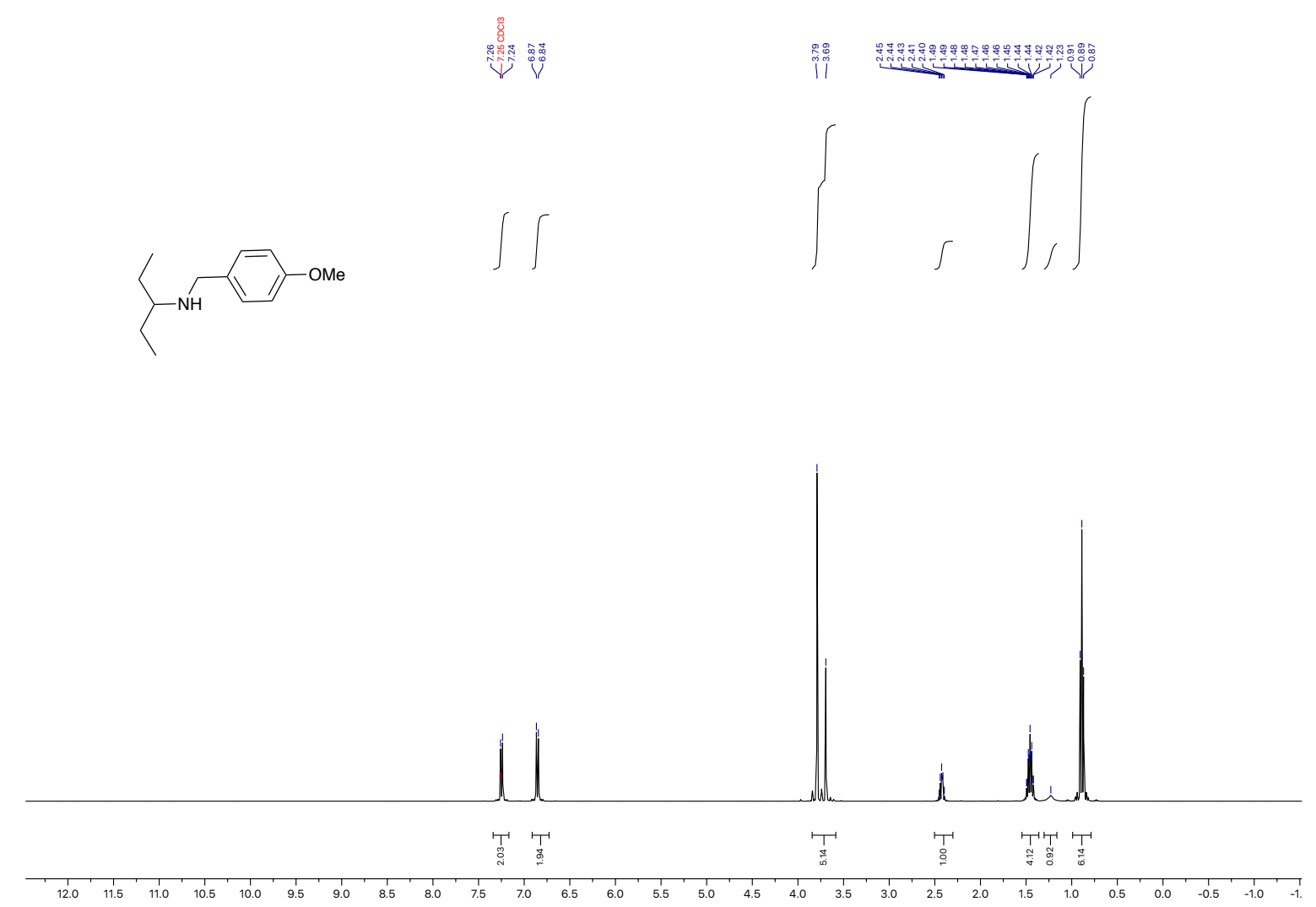

$\underline{300 \mathrm{MHz}^{1} \mathrm{H} \text { NMR of }} \mathbf{N}$-(4-methoxybenzyl)pentan-3-amine (4ib) in $\mathrm{CDCl}_{3}$
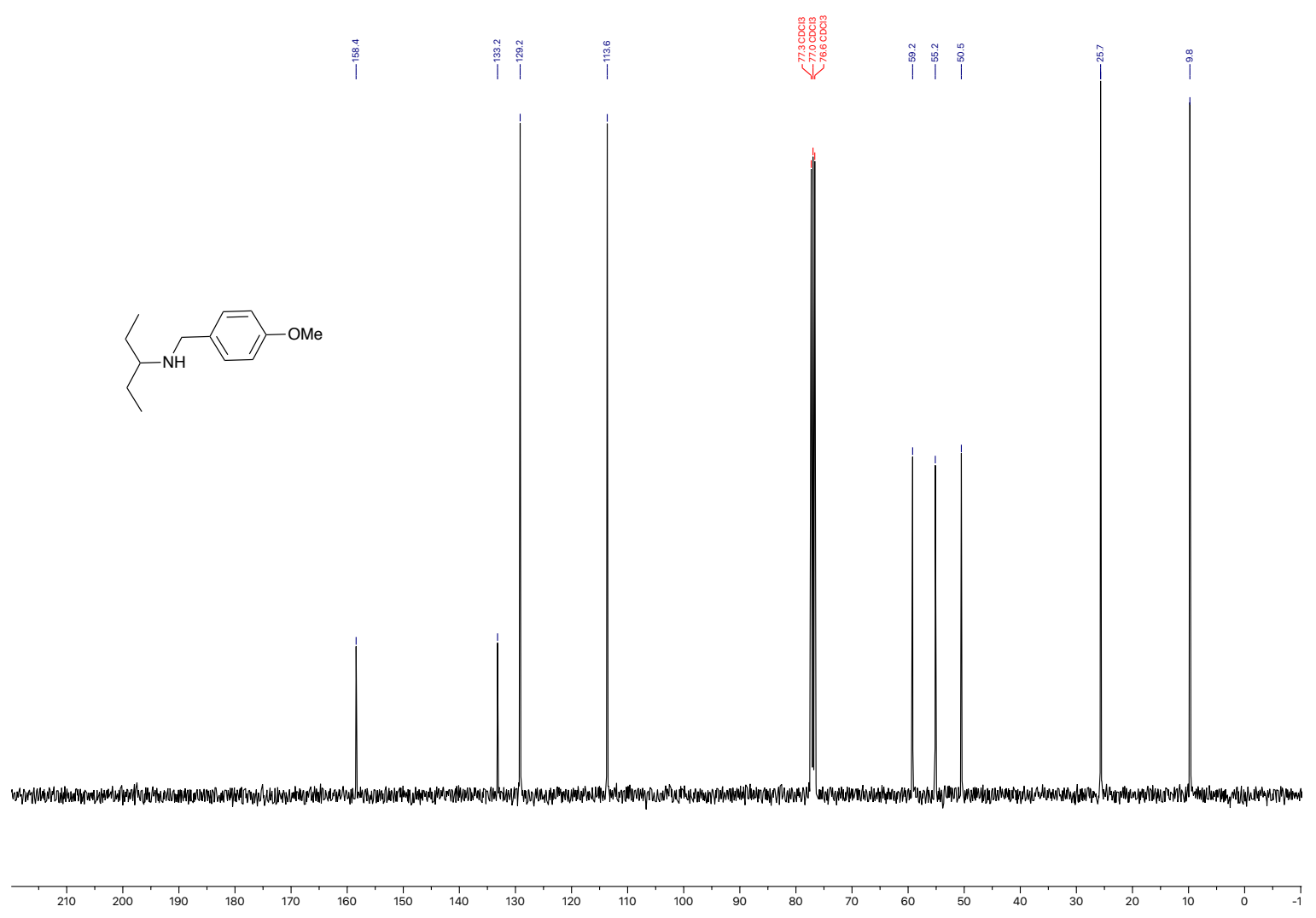

$\underline{75 \mathrm{MHz}^{13} \mathrm{C} \text { NMR }\{\mathrm{H}\} \text { of } \mathrm{N}-(4-m e t h o x y b e n z y l) p e n t a n-3 \text {-amine (4ib) in } \mathrm{CDCl}_{3}}$ 

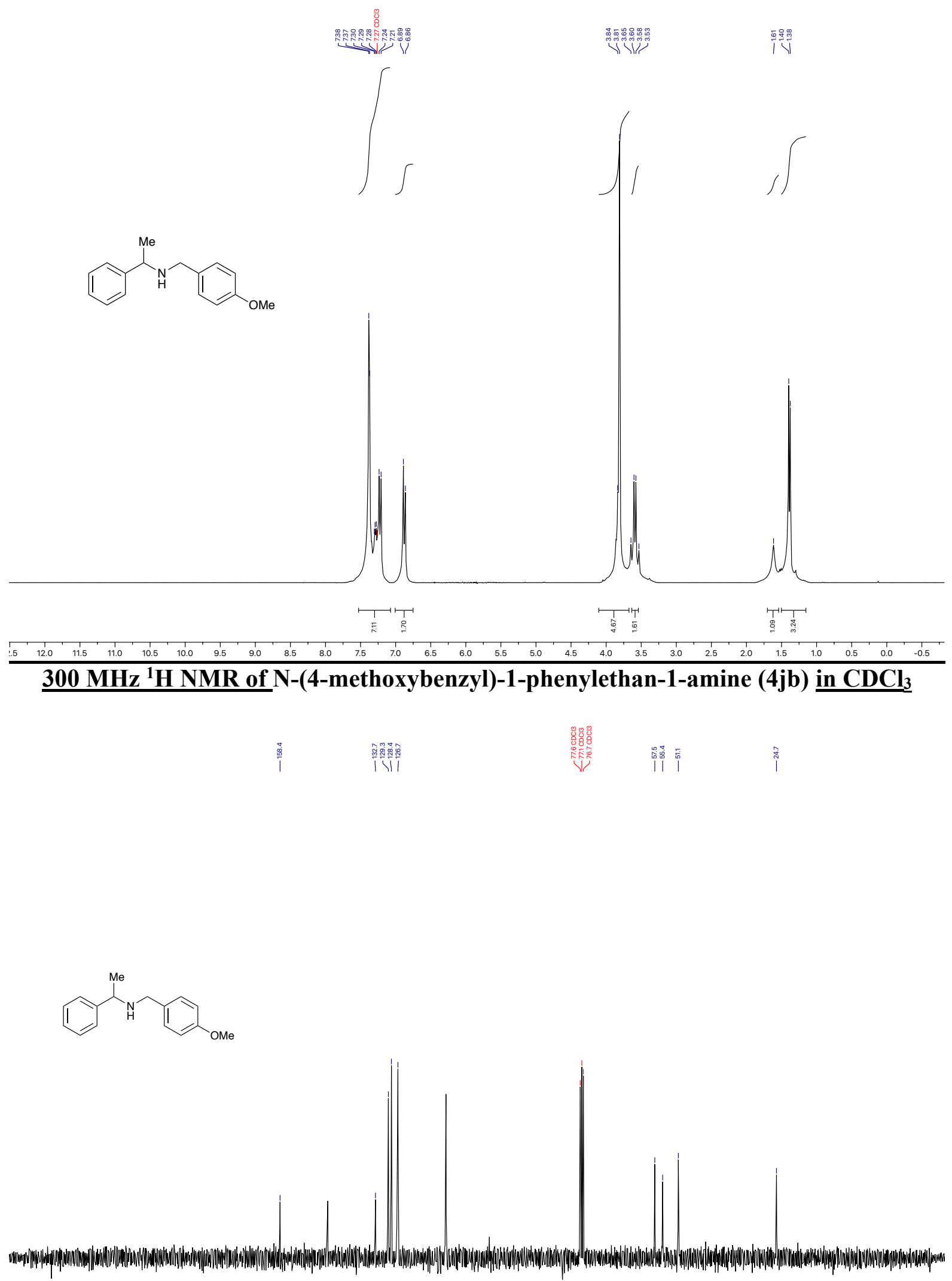

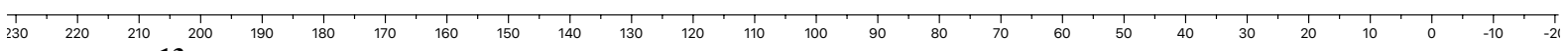
$\underline{75 \mathrm{MHz}}{ }^{13} \mathrm{C}\{\mathrm{H}\}$ NMR of $\mathrm{N}-(4-m e t h o x y b e n z y l)-1-p h e n y l e t h a n-1-a m i n e ~(4 \mathrm{jb}) \underline{\text { in } \mathrm{CDCl}_{3}}$

S31 


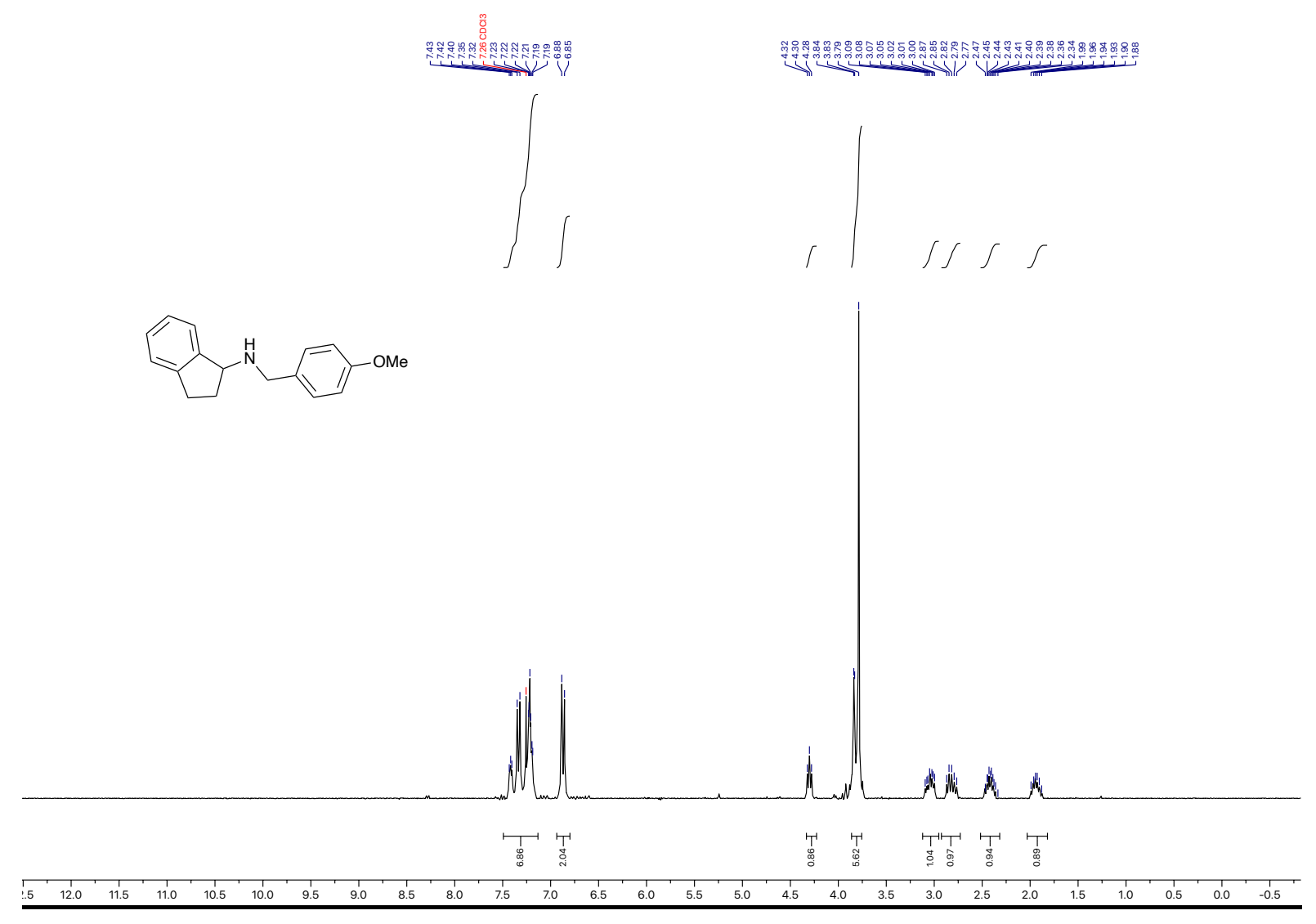

$\underline{300 ~ M H z}{ }^{1} \mathrm{H}$ NMR of $\mathrm{N}-(4-m e t h o x y b e n z y l)-2,3-d i h y d r o-1 H-i n d e n-1-a m i n e ~(4 \mathrm{~kb})$ in

\section{$\underline{\mathrm{CDCl}_{3}}$}
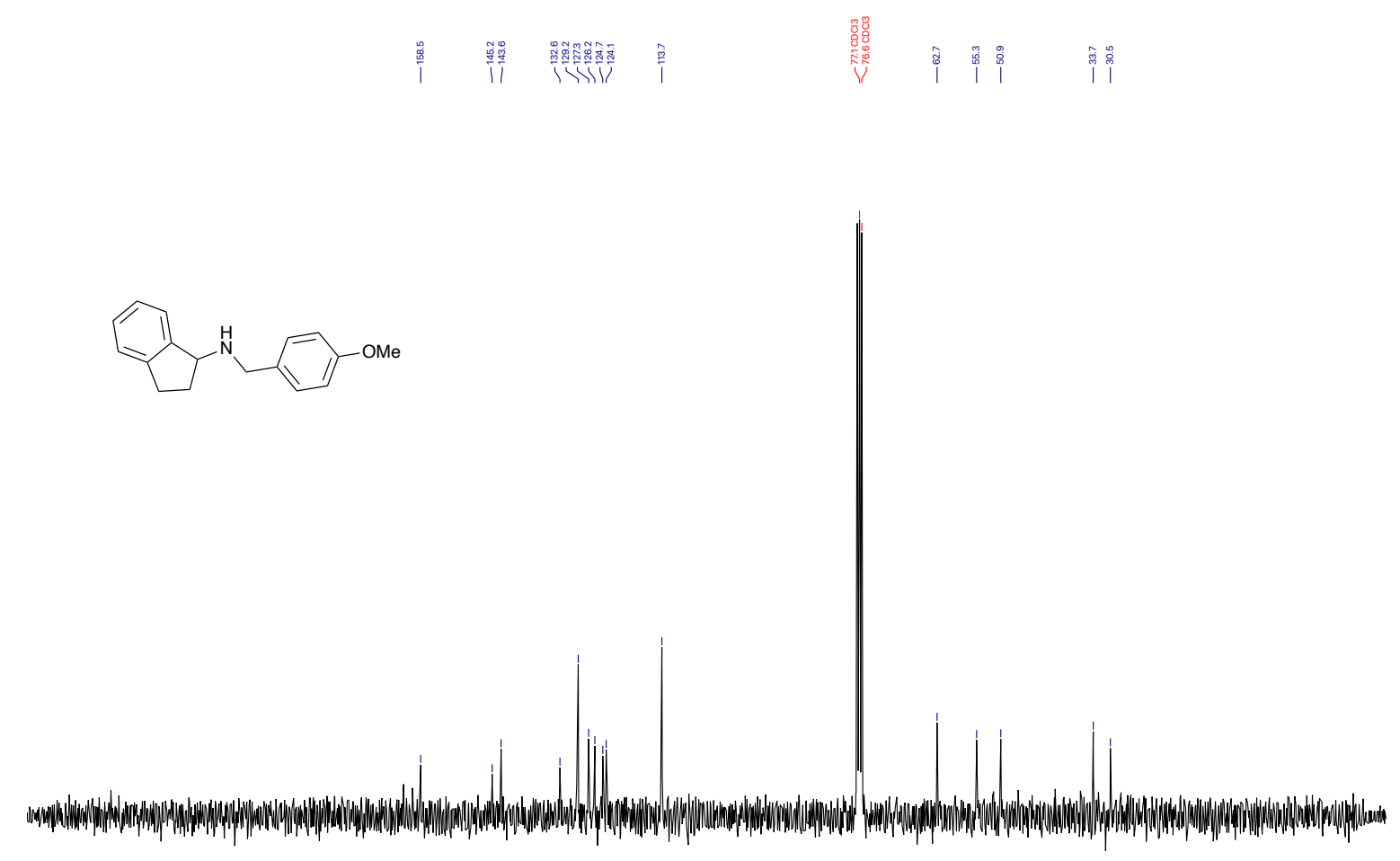

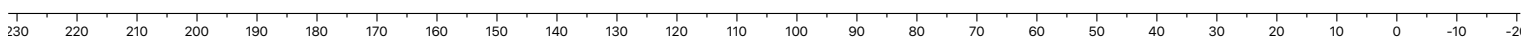

$75 \mathrm{MHz}{ }^{13} \mathrm{C}\{\mathrm{H}\}$ NMR of $\mathrm{N}-(4-m e t h o x y b e n z y l)-2,3-d i h y d r o-1 H-i n d e n-1-a m i n e ~(4 \mathrm{~kb}) \underline{\text { in }}$ $\underline{\mathrm{CDCl}_{3}}$ 


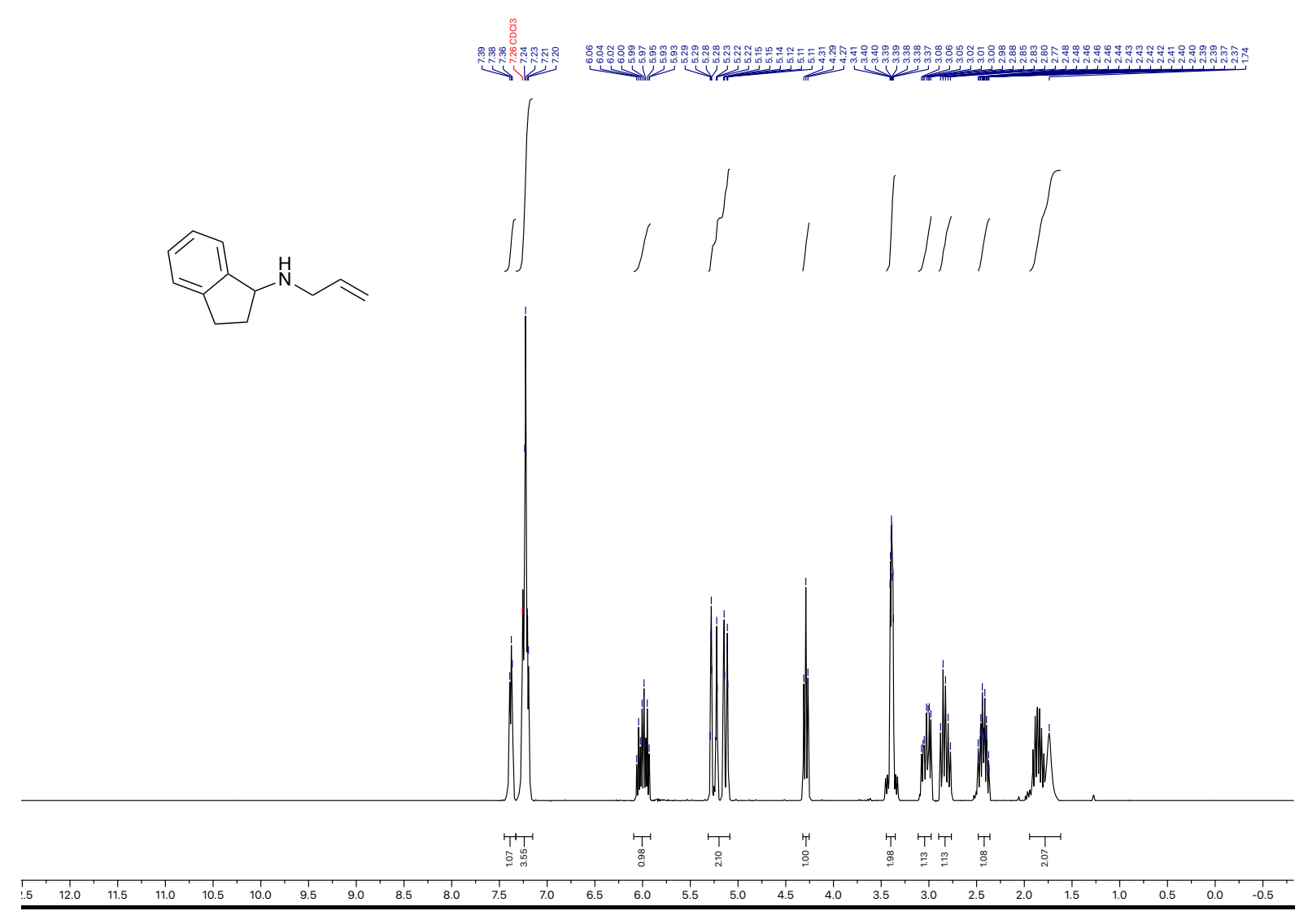

$\underline{300 \mathrm{MHz}}{ }^{1} \mathrm{H}$ NMR of $\mathrm{N}-a l l y l-2,3-d i h y d r o-1 H-i n d e n-1-a m i n e ~(4 k k)$ in $\underline{\mathrm{CDCl}}_{3}$
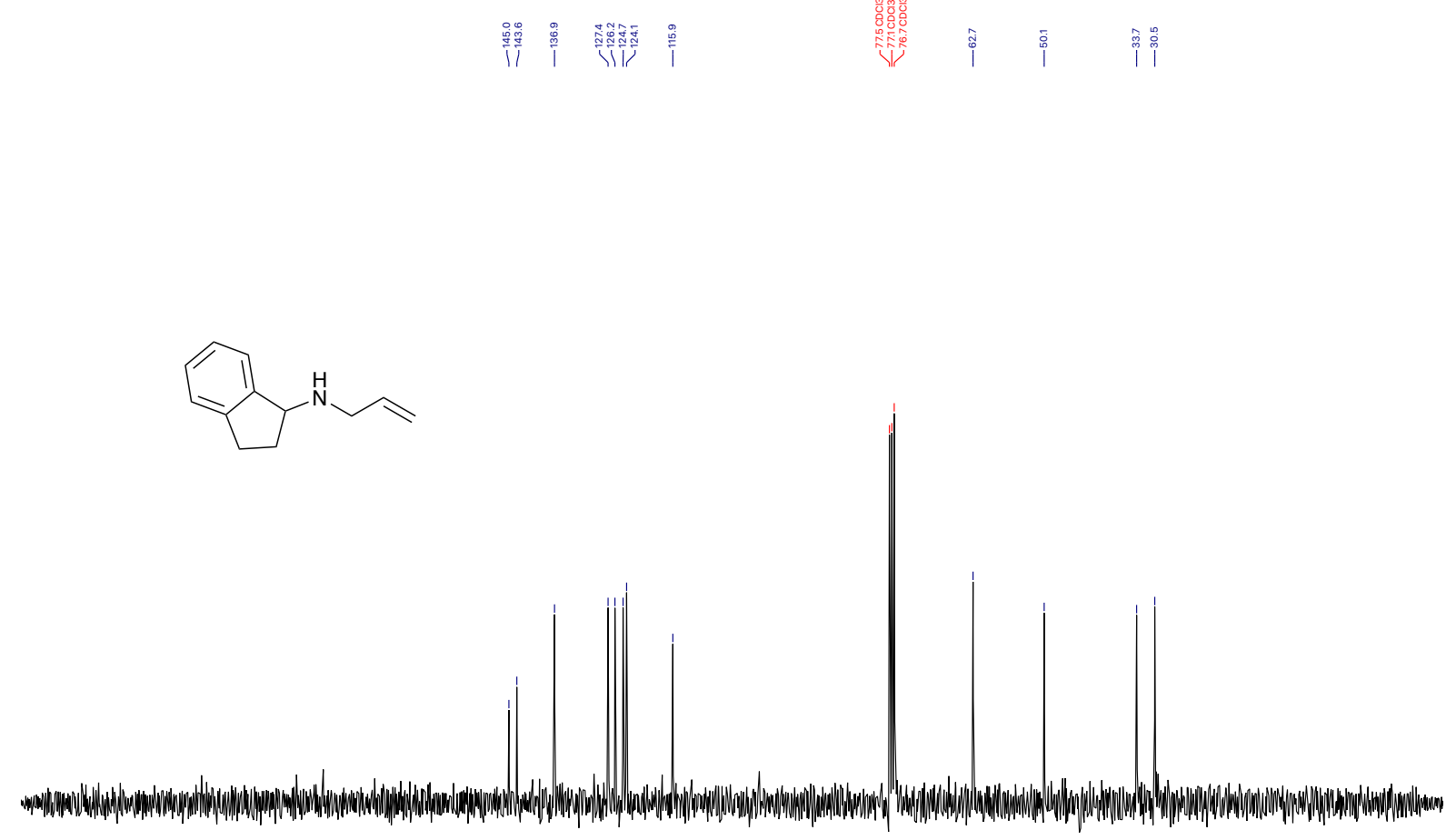

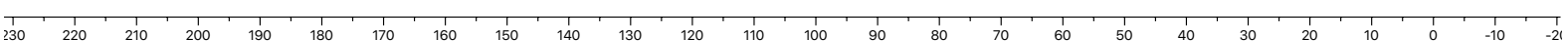
$\underline{75 \mathrm{MHz}}{ }^{13} \mathrm{C}\{\mathrm{H}\}$ NMR of $\mathrm{N}$-allyl-2,3-dihydro-1H-inden-1-amine in (4kk) $\mathrm{CDCl}_{3}$ 


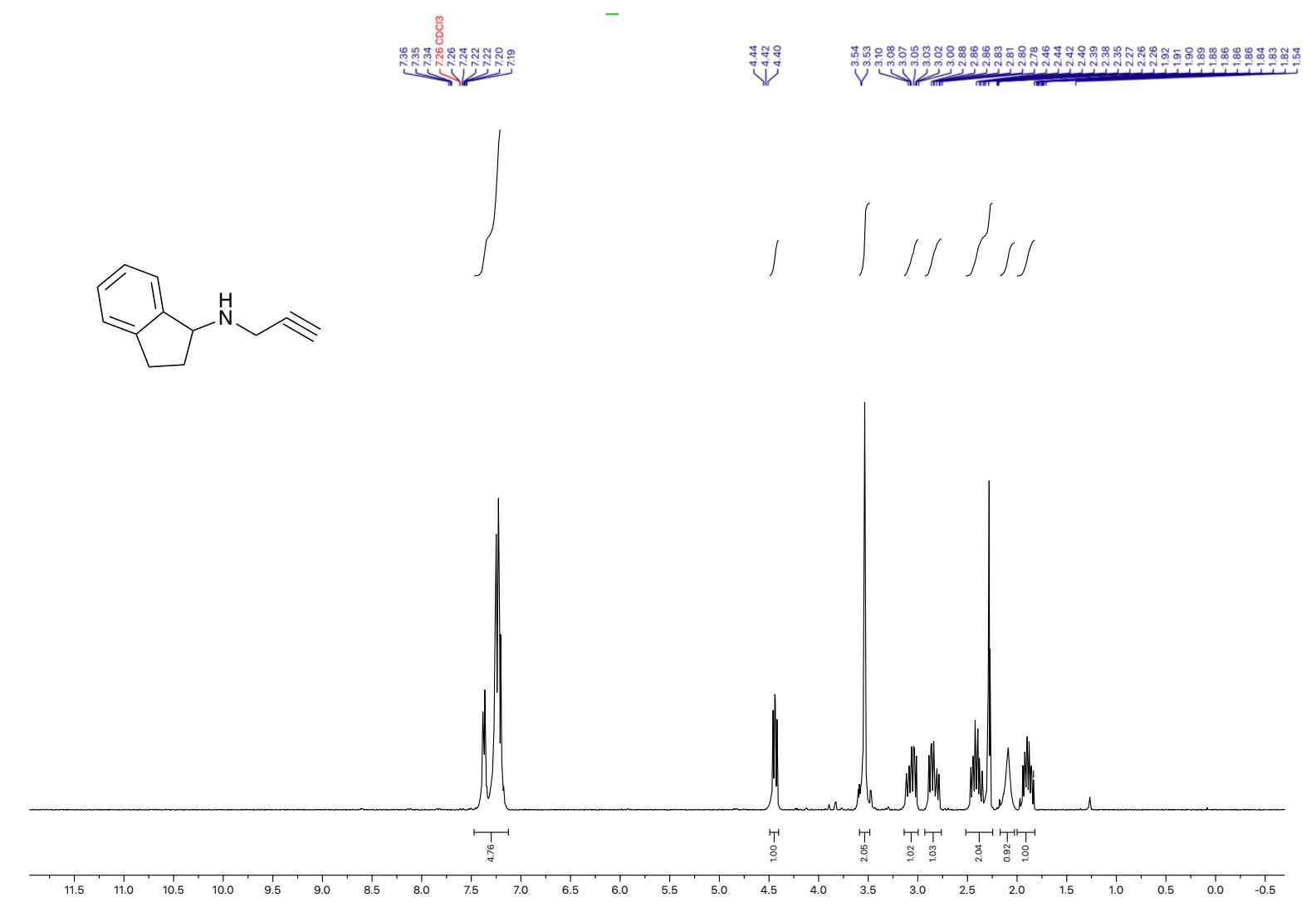

$\underline{300 \mathrm{MHz}}{ }^{1} \mathrm{H}$ NMR of Rasagiline (4kl) in $\mathrm{CDCl}_{3}$

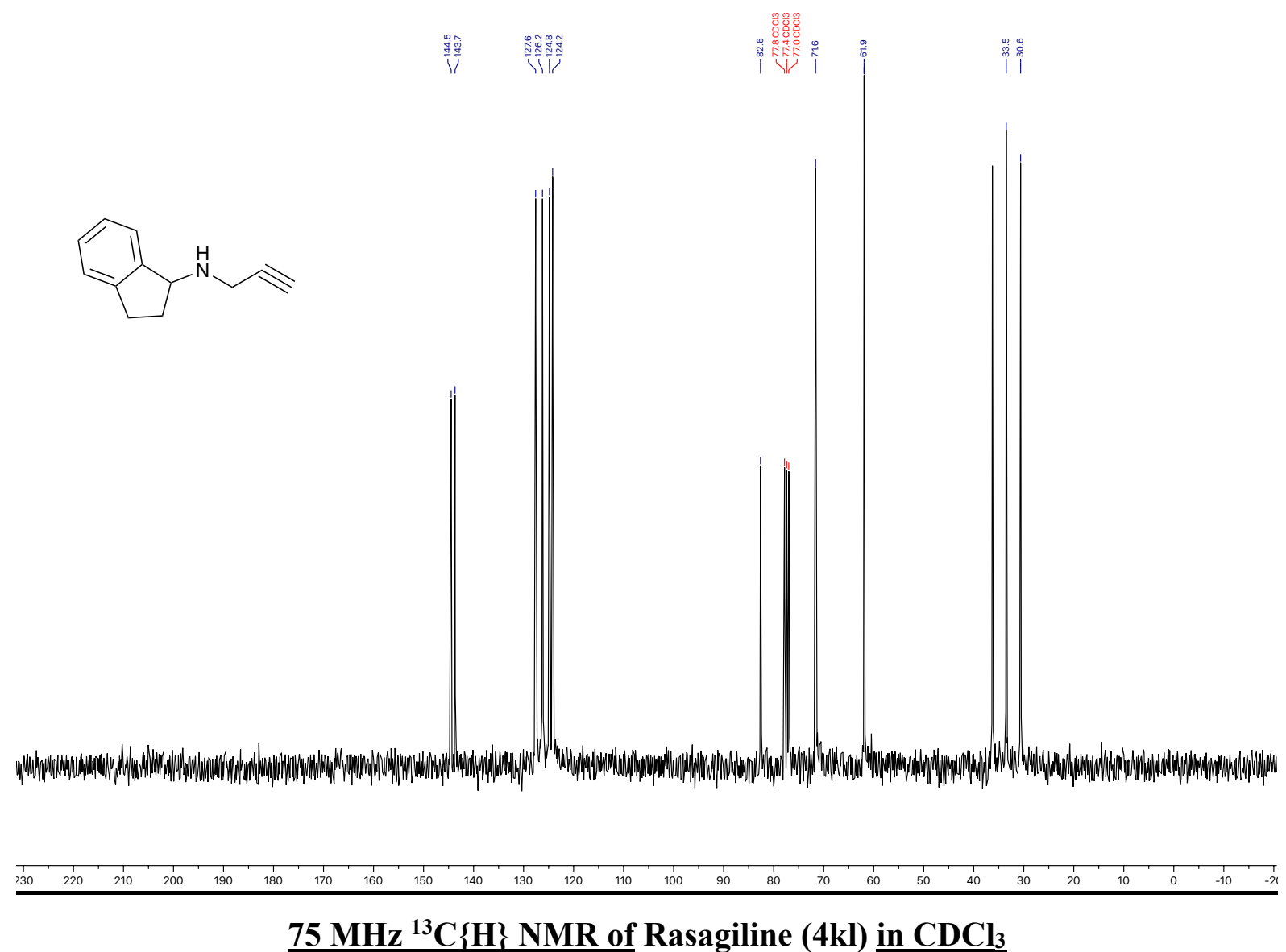


Green Matrix calculation - Process mass intensity (PMI) ${ }^{a}$

$$
P M I=\frac{\sum \text { MassOfMaterials }}{\text { MassOfIsolatedProduct }}
$$

(A) Trimethyl borate catalysed reductive amination

Dibenzyl amine (3aa)<smiles>c1ccc(CNCc2ccccc2)cc1</smiles>

\begin{tabular}{|c|c|c|c|c|c|}
\hline & & $\begin{array}{c}\text { Equivalents } \\
\text { used }\end{array}$ & mmol & MW (g/ mol) & $\mathrm{g}$ \\
\hline \multirow{4}{*}{ Reactants } & Benzaldehyde & 1 & 10 & 106.12 & 1.06 \\
\hline & Benzylamine & 1.1 & 11 & 107.15 & 1.17 \\
\hline & $\mathrm{B}(\mathrm{OMe})_{3}$ & 0.5 & 5 & 103.91 & 0.518 \\
\hline & $\mathrm{NH}_{3} \mathrm{BH}_{3}$ & 1 & 10 & 31 & 0.31 \\
\hline \multirow{5}{*}{ Auxiliary } & $\mathrm{HCl}$ & & & 36.5 & 6.57 \\
\hline & $\mathrm{NaOH}$ & & & 38 & 7.2 \\
\hline & $\mathrm{H}_{2} \mathrm{O}$ & & & 18 & 10 \\
\hline & diethyl ether & & & & 35 \\
\hline & $\mathrm{Na}_{2} \mathrm{SO}_{4}$ & & & & 10 \\
\hline Product & $\begin{array}{c}\text { Dibenzylamine } \\
\text { (4aa) }\end{array}$ & 0.85 & 8.5 & 197.27 & 1.68 \\
\hline \multirow{4}{*}{ Calculation } & $\begin{array}{c}\text { Total mass in } \\
\text { process }\end{array}$ & & & & 73.508 \\
\hline & Mass of product & & & & 1.68 \\
\hline & $\%$ Yield & & & & 85 \\
\hline & PMI & & & & 43.754 \\
\hline
\end{tabular}

${ }^{a}$ Jimenez-Gonzalez, C.; Ponder, C. S.; Broxterman, Q. B.; Manley, J. B. Org Process Res. Dev. 2011, 15, 912-917. 
(B) $\mathrm{Ti}(\mathrm{O}-i-\mathrm{Pr})_{4}$ catalysed reductive amination

Dibenzyl amine (3aa)<smiles>c1ccc(CNCc2ccccc2)cc1</smiles>

\begin{tabular}{|c|c|c|c|c|c|}
\hline & & $\begin{array}{c}\text { Equivalents } \\
\text { used }\end{array}$ & mmol & $\begin{array}{c}\mathrm{MW}(\mathrm{g} / \\
\mathrm{mol})\end{array}$ & $\mathrm{g}$ \\
\hline \multirow{4}{*}{ Reactants } & Benzaldehyde & 1 & 10 & 106.12 & 1.06 \\
\hline & Benzylamine & 1.2 & 12 & 107.15 & 1.28 \\
\hline & $\operatorname{Ti}(\mathrm{O}-i-\mathrm{Pr})_{4}$ & 1.2 & 12 & 284.22 & 3.410 \\
\hline & $\mathrm{NH}_{3} \mathrm{BH}_{3}$ & 1.2 & 12 & 31 & 0.45 \\
\hline \multirow{6}{*}{ Auxiliary } & $\mathrm{HCl}$ & & & 36.5 & 6.57 \\
\hline & THF & & & & 35.6 \\
\hline & $\mathrm{NaOH}$ & & & 38 & 7.2 \\
\hline & $\mathrm{H}_{2} \mathrm{O}$ & & & 18 & 10 \\
\hline & diethyl ether & & & & 35 \\
\hline & $\mathrm{Na}_{2} \mathrm{SO}_{4}$ & & & & 10 \\
\hline \multirow[t]{2}{*}{ Product } & Dibenzylamine & 0.94 & 9.4 & 197.27 & 1.85 \\
\hline & $\begin{array}{c}\text { Total mass in } \\
\text { process }\end{array}$ & & & & 112.42 \\
\hline \multirow[t]{3}{*}{ Calculation } & Mass of product & & & & 1.85 \\
\hline & $\%$ Yield & & & & 94 \\
\hline & PMI & & & & 60.7 \\
\hline
\end{tabular}

